\title{
Energy of fermionic ground states with low-entanglement single-reference expansions, and tensor-based strong-coupling extensions of the coupled-cluster method
}

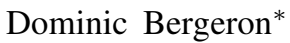 \\ Département de physique, Université de Sherbrooke, Québec, Canada
}

(Dated: January 13, 2020)

\begin{abstract}
We consider a fermionic system for which there exist a single-reference configuration-interaction (CI) expansion of the ground state wave function that converges, albeit not necessarily rapidly, with the increasing number of particle-hole excitations. For such systems, we show that, whenever the coefficients of Slater determinants (SD) with $l \leq k$ excitations can be defined with a number of free parameters $N_{\leq k}$ bounded polynomially in $k$, the ground state energy $E$ only depends on a small fraction of all the wave function parameters, and is the solution of equations of the coupled-cluster (CC) form. This generalizes the standard CC method, for which $N_{\leq k}$ is bounded by a constant. Based on that result and low-rank tensor decompositions (LRTD), we discuss two possible extensions of the CC approach for wave functions with general polynomial bound for $N_{\leq k}$. The most straightforward of those extensions uses the LRTD to represent the amplitudes of the CC cluster operator $T$ which, unlike in the $\mathrm{CC}$ case, is not truncated with respect to number of excitations, and the energy and tensor parameters are given by a LRTD-adapted version of standard CC equations. The LRTD can also be used to directly parametrize the wave function coefficients, which involves different equations of the CC form. We derive those equations for the coefficients of SD's with $l \leq 4$ excitations, using the CC exponential wave function ansatz with a different type of excitation operator, and a representation of the Hamiltonian in terms of excited particle and hole operators. To complete the proposed computational methods, we construct compact tensor representations of coefficients, or $T$-amplitudes, using superpositions of tree tensor networks which take into account different possible types of entanglement between excited particles and holes. Finally, we discuss why the proposed CC extensions are theoretically applicable at larger coupling strengths than those treatable by the standard CC method.
\end{abstract}

\section{Introduction}

The accurate prediction of low temperature properties of many particles system is the main goal of theoretical condensed matter and quantum chemistry research. The quantum nature of those systems however poses a seemingly unsurmountable challenge because of the exponential growth of the problem's complexity with the number of particles. For bosonic systems, quantum Monte Carlo (QMC) methods allow to overcome the exponential complexity. At a fundamental level however, matter is made of fermions, and the computation remains exponentially hard even with QMC methods because of the well known fermionic sign problem due to the anti-commutation of fermion operators $\stackrel{1}{\text {. }}$.

Although no general recipe exists to solve the manyfermions problem, many different methods have been developed to successfully study specific types of molecules or phases of matter using adapted approximations. Those approximations typically become exact either in the weak- or the strong-coupling (interaction) limits. One weak-coupling approach that has been very successful in quantum chemistry is the coupled-cluster (CC) method ${ }^{2.3}$ (review in Ref. 4), which uses the wave function ansatz $\exp (T)|\phi\rangle$, where $|\phi\rangle$ is a reference Slater determinant (SD) playing the role of a vacuum, and $T$ is an excitation operator creating states with different numbers of particle-holes excitations on that vacuum. Unlike the closely related configuration-interaction (CI) method, the $\mathrm{CC}$ method is size-extensive, i.e., the energy scales correctly with system size, which makes it suitable as well for weakly correlated condensed matter systems ${ }^{5}$. Although exact only in the weak-coupling limit, it can however include a reasonable amount of quantum correlations, as compared to density functional theory for instance, but it eventually fails dramatically at strong coupling $\underline{\underline{6}}$. There are also several extensions of the CC method aimed at treating strong correlations, among which are the multireference CC methods (reviewed in Ref. 7), and various single reference extensions that use correlation or projection operators $6,8-12$.

The ability to treat strong correlations in fermionic systems is necessary to model some of the most interesting materials, for instance, Mott insulators, Heavy Fermions systems and high-temperature cuprate superconductors. For spin systems, i.e. very strongly interacting half-filled systems, tensor networks (TN) states have proven very effective (reviews in Refs. 13 and 14). TN methods have also been applied to fermionic systems $5-23$. The main idea behind a TN, which is a type of low-rank tensor decomposition (LRTD), is to take advantage of the small entanglement actually present in the ground state of systems with local Hamiltonians to drastically reduce the number of free parameters required to represent the ground state. TN design therefore relies on the locality of the Hamiltonian $(H)$. Other types of LRTD, which are not TN and do not rely on the locality of $H$, have also been used in quantum chemistry, and CC calculation in particular, to reduce computational complexity ${ }^{24}-32$. In those cases however, the LRTD were used as purely mathematical tools, without reference to the system's entanglement, and without modifying the nature of the CC approximation.

In the present work, we explicitly assume a ground state with low entanglement as in TN methods, but with a singlereference wave function expansion, as in the $\mathrm{CI}$ and $\mathrm{CC}$ methods, and a Hamiltonian assumed to be non-local. We then discuss the use of LRTD not to reduce computational complexity, but to construct different size-extensive approximations than the standard CC ones, in order to treat stronger correlations, 
without using projection or correlation operators.

More specifically, given a single-reference expansion of the ground state of a fermionic system, we first show that the energy of that state is the solution of generalized CC equations whenever the coefficients of SD's with $l \leq k$ particle-hole excitations can be defined with a total number of parameters $N_{\leq k}$ bounded polynomially in $k$. This also implies that the energy depends only on a small fraction of all the wave function parameters and coefficients. We will see that the standard CC approximation corresponds to the simplest of this type of parametrization, where $N_{\leq k}$ is bounded by a constant. We then discuss two other types of parametrization with arbitrary polynomial bound for $N_{\leq k}$ based on LRTD: one in which the LRTD parametrize the amplitudes in the CC cluster operator $T$ and allow to close the CC equations without truncating $T$ on the number of excitations axis, and another in which the LRTD parametrize the wave function coefficients directly. For the latter, we derive generalized CC equations for coefficients of SD's with up to four excitations. To do so, we use a $T$ operator such that $T^{l}|\phi\rangle$ is proportional to the $l$ excitation part of the wave function and $\exp (T)|\phi\rangle$ is the formally exact full CI wave function, and a representation of the Hamiltonian in terms of excited particle and hole operators involving only standard second quantization, instead of the usual CC particle-hole normal ordering notation. For the two proposed approaches, we have to consider higherorder tensors than in the tensor implementations of CC, and for which more complex tensor representations are required to obtain a globally compact low-entanglement representation of the involved coefficients or $T$-amplitudes. For that purpose, we construct representations which can be described as superpositions of tree tensor networks (STTN), and are designed to maximize compactness by taking into account the different possible types of entanglement between excited particles and holes and by sharing tensors between different sets of coefficient. The STTN also allow to estimate the computational complexity of the proposed approaches. The justification for the low-entanglement assumption, despite the non-locality of the Hamiltonian, and the conditions of validity of the CC extensions are also discussed.

The paper is organized as follows: A brief review of the CI and CC methods is given in section II Then, the ground state energy computation result is obtained in section [III. The two types of LRTD parametrization and the choices of orbital basis and reference are discussed in section[IV] Section $\nabla$ describes the particle-hole representation of the Hamiltonian used to derive the generalized $\mathrm{CC}$ equations for the $\mathrm{CI}$ coefficients. Those equations are then presented in section VI] with their derivation provided in appendix and as supplemental material. Finally, the STTN representation is described in section VII followed by a discussion and conclusion in sections VIII and IX, respectively.

\section{The configuration-interaction and coupled-cluster methods}

Let us begin by briefly reviewing the basis of the configuration-interaction and coupled-cluster methods to which we will refer later. More details on those methods can be found in Refs. 4, 33, and 34. First, we define

$$
|\phi\rangle=a_{i_{1}}^{\dagger} a_{i_{2}}^{\dagger} \ldots a_{i_{N}}^{\dagger}|0\rangle
$$

where $a_{i}^{\dagger}$ creates an electron on the spin-orbital $i .|\phi\rangle$ will be called the reference Slater determinant (SD) for the $N$ electrons system and will be used as an approximate vacuum state for the system. We also define

$$
\left|\phi_{j_{1}, j_{2}, \ldots, j_{k}}^{i_{1}, i_{2}, \ldots, i_{k}}\right\rangle=a_{i_{k}}^{\dagger} a_{j_{k}} a_{i_{k-1}}^{\dagger} a_{j_{k-1}} \ldots a_{i_{1}}^{\dagger} a_{j_{1}}|\phi\rangle
$$

a SD with $k$ particle-hole excitations with respect to $|\phi\rangle$. Now, let us write the system's ground state wave function in the Born-Oppenheimer approximation as

$$
\begin{gathered}
|\psi\rangle=|\phi\rangle+\sum_{i, j} c_{j}^{i}\left|\phi_{j}^{i}\right\rangle+\sum_{\substack{\left\langle i_{1}, i_{2}\right\rangle \\
\left\langle j_{1}, j_{2}\right\rangle}} c_{j_{1} j_{2}}^{i_{1} i_{2}}\left|\phi_{j_{1} j_{2}}^{i_{1} i_{2}}\right\rangle \\
+\ldots+\sum_{\substack{\left\langle i_{1} i_{2} \ldots i_{n}\right\rangle \\
\left\langle j_{1}, j_{2} \ldots j_{n}\right\rangle}} c_{j_{1}, j_{2} \ldots j_{n}}^{i_{1} i_{2} \ldots i_{n}}\left|\phi_{j_{1}, j_{2} \ldots j_{n}}^{i_{1} i_{2} \ldots i_{n}}\right\rangle
\end{gathered}
$$

where the $i$ and $j$ indices refer to empty and occupied spinorbitals in $|\phi\rangle$, respectively, $\left\langle i_{1} i_{2} \ldots i_{n}\right\rangle$ is a combination of distinct indices such that $i_{m}<i_{l}$ for $m>l$, and $n \leq n_{\max }=$ $\min \left(N_{e}, N_{o}\right)$, where $N_{e}$ and $N_{o}$ are the number of empty and occupied spin-orbitals, respectively, in $|\phi\rangle$. Expression (3) is the well known configuration-interaction (CI) form of the wave function. In CI calculations, the coefficients are obtained by minimizing the energy $\langle\psi|H| \psi\rangle /\langle\psi \mid \psi\rangle$, where $H$ is the system's Hamiltonian, which is usually done by diagonalizing the matrix representation of $H$ in the $\left|\phi_{j_{1}, j_{2} \ldots j_{k}}^{i_{1} i_{2} \ldots i_{k}}\right\rangle$ basis, where $0 \leq k \leq n$. If $n=n_{\max }$, the method is called full CI or exact diagonalization. Usually $n<n_{\max }$ in the CI method because the entire Hilbert space is too large for the full CI calculation to be tractable.

The single particle orbitals in (1) are typically HartreeFock spin-orbitals. However, the optimal orbitals for CI are called natural spin-orbitals and are the orbitals that diagonalize the single particle density matrix $\left\langle\psi_{F C I}\left|a_{i}^{\dagger} a_{j}\right| \psi_{F C I}\right\rangle$, where $\left|\psi_{F C I}\right\rangle$ is the full CI ground state. They are also the orbitals that minimize the variance $\sum_{i}\left(\left\langle\hat{n}_{i}^{2}\right\rangle-\left\langle\hat{n}_{i}\right\rangle^{2}\right)=$ $\sum_{i}\left\langle\hat{n}_{i}\right\rangle\left(1-\left\langle\hat{n}_{i}\right\rangle\right)\left(\hat{n}_{i}^{2}=\hat{n}_{i}\right.$ for fermions), of the spin-orbital occupation number $\hat{n}_{i}=a_{i}^{\dagger} a_{i}^{33}$, which minimizes the size of the active space, i.e. the number of partially filled spinorbitals, and therefore reduces the size of the Hilbert space for the many-particle problem. However, since $\left|\psi_{F C I}\right\rangle$ is unknown, an approximate $|\psi\rangle$ must necessarily be used in practice, which yields only approximate natural orbitals.

The justification for expanding the wave function in number of excitations with respect to $|\phi\rangle$ is that, because the Hamiltonian has only one- and two-particle terms, it has a blockband-matrix form in such a basis. It is therefore expected that the coefficients of the ground state will decrease rapidly as a function of the number of excitations if the reference SD is well chosen.

The main flaw of the CI method is that it is not size- 
extensive, i.e., the energy of the system does not behave correctly as the system becomes large. For instance, for a homogeneous system of size $V$, instead of being proportional to $V$ as $V \rightarrow \infty$, the CI energy is proportional to $\sqrt{V^{5}}$. This is a consequence of the truncation of Hilbert space, which is equivalent to the inclusion of unlinked diagrams from a perturbation theory perspective, while any size-extensive approximation must only include linked diagrams ${ }^{4,5,35,36}$. The CI method is however a practical method for small molecules and impurity problems such a the Anderson model, one of the cornerstone of Dynamical Mean Field Theory 37,38 .

Instead of truncating Hilbert space at a given number of particle-hole excitations, one can also define a wave function that spans the whole Hilbert space, by using "composite" coefficients above a certain number of excitations. This is what the coupled-cluster (CC) method does by postulating a ground state of the form

$$
|\psi\rangle=e^{T}|\phi\rangle
$$

where $T$ is called the cluster operator and is defined as

$$
T=T_{1}+T_{2}+\ldots+T_{n}
$$

where the $T_{l}$ 's are excitation operators defined as

$$
T_{l}=\sum_{\substack{\left\langle i_{1} i_{2} \ldots i_{l}\right\rangle \\\left\langle j_{1}, j_{2} \ldots j_{l}\right\rangle}} d_{j_{1}, j_{2} \ldots j_{l}}^{i_{1} i_{2} \ldots i_{l}} a_{i_{l}}^{\dagger} a_{j_{l}} a_{i_{l-1}}^{\dagger} a_{j_{l-1}} \ldots a_{i_{1}}^{\dagger} a_{j_{1}} .
$$

When the exponential is expended as a Maclaurin series, the SD's with any number of excitations are present, up to $n_{\max }$. In addition, while the $l \leq n$ excitation coefficients have an irreducible (connected) part, namely an amplitude in $T$, the coefficients of higher order SD's are fully expressed as combinations of products of lower order amplitudes generated by the form $e^{T}$, i.e., they are reducible. For instance, if $n=2$, the single excitation SD's are generated by $T_{1}$ and double excitations SD's are generated by $T_{2}+T_{1}^{2} / 2$, while the three excitations SD's are generated by $T_{1} T_{2}+T_{1}^{3} / 6$. The series (5) is also a type of cumulant expansion, but in excitation operator space with respect to $|\phi\rangle$, i.e., it is the connected part of the operator relating $|\psi\rangle$ to $|\phi\rangle$. For large systems, the $\mathrm{CC}$ ansatz (4) produces much better results than discarding completely the SD's with more than $n$ excitations as in the CI method ${ }^{4}$. On the other hand, the CC method is exact only in the weak coupling limit and fails quite dramatically at strong coupling ${ }^{6}$, which implies that the decoupled expressions for the high order coefficients are bad approximations for the true coefficients in that regime.

If (4) is an eigenstate of the Hamiltonian $H$, we have $H|\psi\rangle=E|\psi\rangle=e^{T} E|\phi\rangle$. Therefore, assuming $|\phi\rangle$ is normalized, since any state $\left|\phi_{j_{1}, j_{2} \ldots j_{l}}^{i_{1} i_{2} \ldots i_{l}}\right\rangle$ is orthogonal to $|\phi\rangle$ for $l \neq 0$, if we project $H|\psi\rangle$ on $\langle\phi| e^{-T}$ or $\left\langle\phi_{j_{1}, j_{2} \ldots j_{l}}^{i_{1} i_{2} \ldots i_{l}}\right| e^{-T}$, we obtain the equations

$$
\begin{aligned}
\left\langle\phi\left|e^{-T} H e^{T}\right| \phi\right\rangle & =E, \\
\left\langle\phi_{j_{1}, j_{2} \ldots j_{l}}^{i_{1} i_{2} \ldots i_{l}}\left|e^{-T} H e^{T}\right| \phi\right\rangle & =0, \quad 1 \leq l \leq n,
\end{aligned}
$$

which, after substitution of $T$ by Eq. (5) and $H$ by its second quantization expression, yields a set of nonlinear equations defining the $T$ amplitudes $d_{j_{1}, j_{2} \ldots j_{l}}^{i_{1} i_{2} \ldots i_{l}}$ for $1 \leq l \leq n$.

By expanding the exponentials in the similaritytransformed Hamiltonian $e^{-T} H e^{T}$, we obtain

$$
\begin{aligned}
& e^{-T} H e^{T}=H+[H, T]+\frac{1}{2}[[H, T], T] \\
& \quad+\frac{1}{3 !}[[[H, T], T], T]+\frac{1}{4 !}[[[[H, T], T], T], T]+\ldots
\end{aligned}
$$

For $T$ given by (5), this series ends after the five terms included above. Very importantly, (7) shows that $e^{-T} H e^{T}$ only contains connected terms because the commutators cancel any disconnected terms. Because of this connectedness of the $\mathrm{CC}$ equations, the $\mathrm{CC}$ energy is size-extensive $e^{4,5,35,36}$. Note also that, because $T$ contains only excitation terms, any term in which it appears on the left of $H$ is disconnected. Consequently,

$$
e^{-T} H e^{T}=\left(H e^{T}\right)_{c}
$$

where the subscript $c$ indicates that only connected terms are kept.

The size-extensivity is a crucial aspect of the CC method, which makes it suitable for large systems, and thus applicable to condensed matter. However, because particle correlations are directly accounted for in the $\mathrm{CC}$ wave function up to two and sometimes three excitations in practice ${ }^{4}$, while higher order coefficients are decoupled, important strong-coupling physics cannot be properly treated. For example, for a SD with more than $n$ excitations and two excited particles or holes with opposite spins occupying an orbital where the Coulomb repulsion is strong, the coefficient should be small in order to minimize the energy, but in most terms defining the $l>n$ excitations coefficients of the $\mathrm{CC}$ wave function, the strongly interacting particles or holes are decoupled, and thus do not include the effect of that repulsion. If all the higher order coefficients were to be negligibly small, this effect would be small. However, on the contrary, the convergence rate of the singlereference expansion (3) decreases as the coupling strength increases because many different SD's become nearly degenerate, and thus have comparable contributions in the ground state. At larger coupling, it therefore becomes more important to control higher order coefficients. Unfortunately, the computational complexity increases exponentially with the truncation order $n$ in the CC method. Other strategies are thus required to treat more strongly correlated systems.

\section{Energy of ground states with low entanglement CI expansions}

Let us consider a system of $N=N_{\uparrow}+N_{\downarrow}$ fermions, for which the CI form of the wave function, Eq. (3), converges, though not necessarily rapidly. In the following, we will show that, when the $l \leq k$ excitation coefficients can be defined with a number of free parameters $N_{\leq k}^{T D}$ bounded above by a 
polynomial in $k$, then the grounds state energy $E$ is the solution of a set of equations of the CC form, Eqs. (6), but where $T$ is generally different from (5). Consequently, $E$ also depends only on a small fraction of all the wave function parameters and wave function coefficients. Note that the convergence condition only serves to ensure that $E$ is the true ground state energy, hence the assumption that the convergence can be slow. This is an important aspect of that result that will be discussed further below.

Before we begin with the proof, let us give a few definitions about tensors. First, the term "order" will be used to specify the number of indices of a tensor. Then, the term "rank" will be used in a similar sense as for matrices, where it is the number of linearly independent columns, or rows, i.e., the number of non-zero singular values of the matrix. However, for tensors, there are more than one definition of rank. First, if we define a simple tensor as the tensor product of vectors, we can represent any tensor as a sum of simple tensors. Then, the simplest definition of a tensor rank is the minimal number of simple tensor in that representation. Another definition is the multilinear rank, or multirank. For a tensor of order $k$, the multirank is the vector $\left(r_{1}, r_{2}, \ldots, r_{k}\right)$ corresponding to the dimensions of the core tensor in the higher order singular value decomposition (HOSVD) ${ }^{39}$. In the following, we use the expression "full rank" in the sense that a tensor does not have a more compact representation than as a multidimensional array of the same dimensions as the original, and "low rank" in the general sense that it can be represented by a tensor product involving less free parameters than the number of elements in the tensor.

To prove the result stated in the first paragraph in the most general case, we first define the following excitation operator T

$$
T=\sum_{i, j} a_{i}^{\dagger} a_{j} \hat{D}_{j}^{i}
$$

where $\hat{D}_{j}^{i}$ is an operator defined as

$$
\hat{D}_{j_{k}}^{i_{k}}\left|\phi_{j_{1} j_{2} \ldots j_{k-1}}^{i_{1} i_{2} \ldots i_{k-1}}\right\rangle=\frac{1}{k} \frac{c_{j_{1} j_{2} \ldots j_{k}}^{i_{1} i_{2} \ldots i_{k}}}{k} c_{j_{1} j_{2} \ldots j_{k-1}}^{i_{1} i_{2} \ldots i_{k-1}}\left|\phi_{j_{1} j_{2} \ldots j_{k-1}}^{i_{1} i_{2} \ldots i_{k-1}}\right\rangle .
$$

where the coefficients are assumed as those of the full CI wave function, Eq. (3). We could write explicitly the operator $\hat{D}_{j}^{i}$ in terms of products of particle number operators, but the def- inition $(9 \mathrm{~b})$ is sufficient. With this definition of $T$, we have

$$
\begin{aligned}
T|\phi\rangle & =\sum_{i, j} a_{i}^{\dagger} a_{j} \hat{D}_{j}^{i}|\phi\rangle \\
& =\sum_{i, j} c_{j}^{i}\left|\phi_{j}^{i}\right\rangle \\
T^{2}|\phi\rangle & =\sum_{i_{2} j_{2}} a_{i_{2}}^{\dagger} a_{j_{2}} \hat{D}_{j_{2}}^{i_{2}} \sum_{i_{1} j_{1}} c_{j_{1}}^{i_{1}}\left|\phi_{j_{1}}^{i_{1}}\right\rangle \\
& =\frac{1}{2} \sum_{i_{1}, i_{2}} c_{j_{1} j_{2}}^{i_{1} i_{2}}\left|\phi_{j_{1} j_{2}}^{i_{1} i_{2}}\right\rangle \\
& \vdots \\
T^{k}|\phi\rangle & =\frac{1}{k !} \sum_{\sum_{j_{1}, j_{2}, \ldots, j_{k}}} c_{j_{1}, j_{2}, \ldots, j_{k}}^{i_{1}, i_{2} \ldots i_{k}}\left|\phi_{j_{1}, j_{2}, \ldots, j_{k}}^{i_{1}, i_{2} \ldots i_{k}}\right\rangle .
\end{aligned}
$$

The definition (9) assumes that, if a coefficient $c_{j_{1}, j_{2}, \ldots, j_{k}}^{i_{1}, i_{2} \ldots i_{k}}$ is finite, all the coefficients which upper and lower indices are subsets of $\left\{i_{1}, i_{2} \ldots i_{k}\right\}$ and $\left\{j_{1}, j_{2}, \ldots, j_{k}\right\}$, respectively, are also finite. They can however be arbitrarily small.

Using (9) in the CC ansatz (4) yields

$$
\begin{aligned}
& e^{T}|\phi\rangle=\left(1+T+\frac{T^{2}}{2}+\frac{T^{3}}{3 !}+\ldots\right)|\phi\rangle \\
& =|\phi\rangle+\sum_{i, j} c_{j}^{i}\left|\phi_{j}^{i}\right\rangle+\frac{1}{4} \sum_{\substack{i_{1}, i_{2} \\
j_{1}, j_{2}}} c_{j_{1} j_{2}}^{i_{1} i_{2}}\left|\phi_{j_{1} j_{2}}^{i_{1} i_{2}}\right\rangle+\ldots \\
& +\left(\frac{1}{n_{\max } !}\right)^{2} \sum_{\substack{i_{1} i_{2} \ldots i_{n_{\max }} \\
j_{1}, j_{2} \ldots j_{n_{\max }}}} c_{j_{1}, j_{2} \ldots j_{n_{\max }}}^{i_{1} i_{2} \ldots i_{\text {max }}}\left|\phi_{j_{1}, j_{2} \ldots j_{n_{\max }}}^{i_{1} i_{2} \ldots i_{n_{\max }}}\right\rangle \\
& =|\phi\rangle+\sum_{i, j} c_{j}^{i}\left|\phi_{j}^{i}\right\rangle+\sum_{\substack{\left\langle i_{1}, i_{2}\right\rangle \\
\left\langle j_{1}, j_{2}\right\rangle}} c_{j_{1} j_{2}}^{i_{1} i_{2}}\left|\phi_{j_{1} j_{2}}^{i_{1} i_{2}}\right\rangle \\
& +\ldots+\sum_{\substack{\left\langle i_{1} i_{2} \ldots i_{n_{\max }}\right\rangle \\
\left\langle j_{1}, j_{2} \ldots j_{n_{\max }}\right\rangle}} c_{j_{1}, j_{2} \ldots j_{n_{\max }}}^{i_{1} i_{2} \ldots i_{\max }}\left|\phi_{j_{1}, j_{2} \ldots j_{n_{\max }}}^{i_{1} i_{2} \ldots i_{n_{\max }}}\right\rangle \\
& =\left|\psi_{F C I}\right\rangle
\end{aligned}
$$

where we have used the fact that $c_{j_{1}, j_{2} \ldots j_{n}}^{i_{1} i_{2} \ldots i_{n}}\left|\phi_{j_{1}, j_{2} \ldots j_{n}}^{i_{1} i_{2} \ldots i_{n}}\right\rangle$ is invariant under the $(n !)^{2}$ different permutations of the indices. The operator (9) therefore allows to put the full CI form of the wave function in the $\mathrm{CC}$ form. The bijective relation between $T^{k}$ and the $k$ excitations part of the wave function will also be useful below.

Now, with $T$ given by (9), the series (7) has $n_{\max }+1$ terms, unlike the CC case for which the number of terms is 5 . Thus, if we expand the exponentials as Maclaurin series in the CC 
equations (6), we obtain

$$
\begin{aligned}
& E \delta_{l 0}=\left\langle\phi_{j_{1}, j_{2} \ldots j_{l}}^{i_{1} i_{2} \ldots i_{l}}\left|e^{-T} H e^{T}\right| \phi\right\rangle \\
&=\left\langle\phi_{j_{1}, j_{2} \ldots j_{l}}^{i_{1} i_{2} \ldots i_{l}}\right|\left(1-T+\frac{T^{2}}{2}-\frac{T^{3}}{3 !}+\ldots+(-1)^{l} \frac{T^{l}}{l !}\right) \\
& \times H\left(1+T+\frac{T^{2}}{2}+\frac{T^{3}}{3 !}+\ldots+\frac{T^{l+2}}{(l+2) !}\right)|\phi\rangle, \\
& 0 \leq l \leq n,
\end{aligned}
$$

where $\delta_{l 0}$ is the Kronecker delta function and $\left\langle\phi_{j_{1}, j_{2} \ldots j_{0}}^{i_{1} i_{2} \ldots i_{0}}\right|=$ $\langle\phi|$. From Eq. (10), those equations depend on the coefficients of the $1 \leq l \leq n+2$ excitation SD's in (11). The maximum power of $T$ on the right-hand side of $H$ is $l+2$ because $H$ can annihilate two particle-hole pairs at most and the maximum power of $T$ on the left-hand side of $H$ is $l$ since the smallest number of excitations is zero. Equations (12) are exact since $e^{T}|\phi\rangle$ is the full CI wave function and thus $H e^{T}|\phi\rangle=E e^{T}|\phi\rangle$ holds exactly.

The number of SD's with $l$ excitations increases nearly exponentially with $l$. Therefore, since equations (12) depend on the $1 \leq l \leq n+2$ excitation coefficients, while the number of equations is equal to the number of SD's with $0 \leq l \leq n$ excitations, the number of parameters is much larger than the number of equations if the equations are truncated at a given $n<n_{\max }$ and the coefficients have full rank. As described in section [I] the CC strategy to close the equations amounts to expressing the coefficients of SD's with $l>n$ excitations using only cluster operator amplitudes for $l \leq n$ excitations. However, this is only one of many possible ways of closing the equations. Indeed, Eqs. (12) can be closed at a value $n<n_{\max }$ if the number of free parameters defining the coefficients increases at a slower rate with the number of excitations than the number of SD's itself. This might seem like a strange assumption, but it is possible if there exist low-rank decompositions of each set of coefficients $\left\{c_{j_{1}, j_{2} \ldots j_{l}}^{i_{1} i_{2} \ldots i_{l}}\right\}$, when the latter is interpreted as a tensor. In practice, the increasing rate of the number of free parameters with $l$ must be slow enough that the equations are closed at $n$ small, so that the number of equations and free parameters are computationally tractable. This is the case if we assume an increasing rate smaller than some low order polynomial. Let us see this in more details.

If the number of up and down spin-orbitals are both equal to $L$, the number of coefficients of the form $c_{j_{1}, j_{2} \ldots j_{k}}^{i_{1} i_{2} \ldots i_{k}}$, assuming that indices $i_{1}$ to $i_{p}$ have spins up and indices $i_{p+1}$ to $i_{k}$ have spins down, is

$$
\begin{aligned}
N_{k, p}^{C I}= & \left(\begin{array}{c}
L-N_{\uparrow} \\
p
\end{array}\right)\left(\begin{array}{c}
L-N_{\downarrow} \\
k-p
\end{array}\right)\left(\begin{array}{c}
N_{\uparrow} \\
p
\end{array}\right)\left(\begin{array}{c}
N_{\downarrow} \\
k-p
\end{array}\right) \\
= & \frac{\left(L-N_{\uparrow}\right) !}{p !\left(L-N_{\uparrow}-p\right) !} \frac{\left(L-N_{\uparrow}\right) !}{(k-p) !\left(L-N_{\uparrow}-k+p\right) !} \\
& \times \frac{N_{\uparrow} !}{p !\left(N_{\uparrow}-p\right) !} \frac{N_{\uparrow} !}{(k-p) !\left(N_{\uparrow}-k+p\right) !} .
\end{aligned}
$$

the number of coefficients with $k$ excitations is

$$
N_{k}^{C I}=\sum_{p=\max \left(0, k-N_{\downarrow}\right)}^{\min \left(k, N_{\uparrow}\right)} N_{k, p}^{C I}
$$

and the total number of coefficients, or CC equations, with up to $k$ excitations is

$$
N_{\leq k}^{C I}=\sum_{l=0}^{k} N_{l}^{C I} .
$$

For $k$ fixed, $N_{\leq k}^{C I}$ is polynomial in $N=N_{\uparrow}+N_{\downarrow}$.

Now, using the fact that

$$
\left(\begin{array}{c}
l \\
m
\end{array}\right) \geq\left(\frac{l}{m}\right)^{m}, \quad 1 \leq m \leq l,
$$

we have that

$N_{k, p}^{C I} \geq\left(\frac{L-N_{\uparrow}}{p}\right)^{p}\left(\frac{L-N_{\downarrow}}{k-p}\right)^{k-p}\left(\frac{N_{\uparrow}}{p}\right)^{p}\left(\frac{N_{\downarrow}}{k-p}\right)^{k-p}$

If we consider only the cases $p=k$,

$$
N_{k, k}^{C I} \geq\left(\frac{\left(L-N_{\uparrow}\right) N_{\uparrow}}{k^{2}}\right)^{k} .
$$

Now, if the number of parameters $N_{\leq k}^{T D}$ defining the $l \leq k$ excitation coefficients is bounded above polynomially in $k$, we have $N_{\leq k+2}^{T D} \leq a k^{r}$, where $r \in \mathbb{N}$ and $a>0$. We then have

$$
\begin{aligned}
& \frac{N_{\leq k}^{C I}}{N_{\leq k+2}^{T D}}>\frac{N_{k, k}^{C I}}{N_{\leq k+2}^{T D}} \geq \frac{\left(\frac{\left(L-N_{\uparrow}\right) N_{\uparrow}}{k^{2}}\right)^{k}}{a k^{r}} \\
& \frac{N_{\leq k}^{C I}}{N_{\leq k+2}^{T D}}>\frac{1}{a} e^{k \ln \frac{\left(L-N_{\uparrow}\right) N_{\uparrow}}{k^{2}}-r \ln k}
\end{aligned}
$$

For $k^{2} \ll\left(L-N_{\uparrow}\right) N_{\uparrow}$, this ratio grows rapidly with $k$ and thus, for $r$ small, there is a small value of $k$ for which $N_{\leq k}^{C I}>N_{\leq k+2}^{T D}$. Therefore, given the polynomially bounded parametrization of the coefficients, the equations (12) are closed at a small value of $n$ and the ground state energy $E$ depends only on the $k \leq n+2$ excitation coefficients, a fraction of order $\left(\frac{n}{N}\right)^{r}$ or smaller of the total number of free parameters defining the wave function, and $E$ can be computed in polynomial time by solving a set of equations of the CC form, or generalized CC equations. This concludes the proof of the result stated in the first paragraph of the present section.

A corollary to that computation result is that equal-time correlation functions at zero temperature can also be computed if the ground state energy can. This is the Hellmann-Feynman theorem, which is proved in Appendix $\mathrm{A}$. In particular, this fact can be used to determine an optimal spin-orbital basis. This is discussed further in section IVC

The standard CC method corresponds to the simplest application of the computation result we have just proved. Indeed, 
since the $l>n$ excitation coefficients depend only on low order amplitudes in CC, $N_{\leq k}^{C C}$ is constant for $k \geq n$, i.e., it is the simplest possible polynomial.

The fact that there is no lower bound on the convergence rate of the $\mathrm{CI}$ expansion in the above result implies that it also applies to strongly correlated systems. Indeed, as discussed at the end of section $\Pi$ at strong, but finite, coupling strength, the ground state of such systems also possess a converging single-reference CI expansion. The difference with the weak coupling regime is that the expansion converges only slowly as the number of excitations increases, hence the failure of approximations that truncate the cluster operator with respect to that parameter. As discussed in section IV that follows, the above result allows to use CC equations with other types of approximations much better suited to the strongly correlated regime.

As mentioned above, a polynomially bounded parametrization of the sets of coefficients $\left\{c_{j_{1}, j_{2} \ldots j_{l}}^{i_{1} i_{2} \ldots i_{l}}\right\}$ for $1 \leq l \leq n+2$ can be obtained using low-rank tensor decompositions, which are based on the existence of basis transformations, in single and multiple particle and hole spaces, such that a superposition of $l$ excitations SD's can be written with a much smaller number of components in the transformed basis. Since the rank can be used as a measure of entanglement, this type of parametrization is also a low-entanglement representation of the coefficients. In section IV that follows, we will discuss two types of LRTD-based parametrization which are applications of the ground state energy computation result given above, and are good candidates for strong-coupling extensions of the CC method. Adapted LRTD-based representations applicable in both cases will also be described in sectionVII

Note that equations (12) are also valid if $T$ is the CC cluster operator, Eq. (5), but without any truncation. Then, the maximum power of $T$ is 4 , as discussed in section $\amalg$ and only the terms of $T$ up to $T_{l}$ contribute on the left-hand side of $H$ and up to $T_{l+2}$ on the right-hand side. However, as shall be discussed in section IV that follows, that choice produces a particular type of approximation when combined with the LRTD-based representations. On the other hand, thanks to the operator (9), we obtain a result applicable to any converging single-reference expansion with a polynomially bounded parametrization of the wave function coefficients, which includes the CC approximation and the two types of approximations discussed in the section IV

\section{Extending the coupled-cluster method with low-rank tensor decompositions}

In this section we discuss two possible generalizations of the CC method based the result of section [II] The first one is a straightforward extension of $\mathrm{CC}$ and the second approach uses a parametrization more directly connected to the CI form of the wave function. We will also discuss the choice of spinorbital basis and reference SD.

\section{A. A coupled-cluster approach without truncation of the cluster operator}

The simplest tensor-based extension of CC, or TCC for "tensor-CC", that uses a general polynomially bounded parametrization of the wave function coefficients applies the LRTD-based parametrization to the amplitudes $d_{j_{1}, j_{2} \ldots j_{k}}^{i_{1} i_{2} \ldots i_{k}}$ in the CC cluster operator, Eq. (5), for all the terms with $1 \leq k \leq n+2$ involved in the equations (12) with $0 \leq l \leq n$. The explicit form of those equations in terms of cluster operator amplitudes and Hamilitonian parameters are the usual CC equations, except that $T$ is not truncated with respect to excitation number, or equivalently, truncated at $n+2$ excitations. Therefore, if $n=2$, we use only the CC equations with $l \leq 2$ of the CC approximation with truncation of $T$ at four excitations (CCSDTQ). To complete the equations, the LRTD-based representation for sets of coefficients with $1 \leq k \leq 4$ excitations provided in section VII, or other similar representations, can be used to parametrize the amplitudes.

The most crucial aspect of that approximation, as compared with standard CC, is the fact that there is no truncation of $T$ with respect to excitation number. Indeed, if we consider the wave function coefficients generated by the CC exponential ansatz with such a $T$ operator, the reducible parts then have irreducible corrections at all orders, except that those corrections are expressed with LRTD. For instance, this type of parametrization of the $T$ amplitudes can take into account local correlations, which can have a similar effect as a Gutzwiller operator ${ }^{40}$ that modulates the coefficients of SD's with strongly correlated excited quasiparticles (i.e. particles or holes) occupying the same spatial orbital. This is only the simplest possibility allowed by such a parametrization, since non-local correlations can be treated as well. Therefore, in principle, this type of tensor-based approximation yields results valid at stronger coupling than standard CC. In fact, while the CC approximation is exact in the weak coupling limit, corresponding to a fast converging cluster operator, TCC would be exact in the low-entanglement limit of the cluster operator $T$, which includes the weak coupling limit, but is more general. It would therefore be a very compact approximation at weak coupling, but also at moderately strong coupling, as long as the wave function coefficients can be represented by the reducible parts generated by the exponential ansatz, corrected by irreducible LRTD-based terms. On the other hand, as the coupling strength increases, the reducible parts become worse approximations of the actual wave function coefficients, which requires larger and more complex irreducible parts that can become difficult to represent using LRTD. The type of approximation discussed in section IVB that follows could then become useful.

\section{B. Computing CI coefficients using generalized $\mathrm{CC}$ equations}

Another possible tensor-based extension of the $\mathrm{CC}$ method uses the definition (9) for $T$ and equations (12). This produces equations similar to the $\mathrm{CC}$ ones, though involving the $\mathrm{CI}$ coefficients instead of cluster operator amplitudes, which are 
closed by parametrizing the coefficients with LRTD. This approach could thus be called TCICC, for "tensor-CI using CC equations". In that case, unlike the approximation described in section IVA above, the wave function coefficients are completely defined using LRTD and do not have reducible parts. Although such an approximation is probably not optimal at weak coupling, it is in principle applicable at very strong coupling, as long as there exist a single-reference CI representation of the wave function that converges at least slowly with the number of particle-hole excitation, ensuring that a good approximation to the actual ground state energy can be found. Such an approximation is exact in the low-entanglement limit of the CI coefficients, which is very different from the limit of validity of the CC approximation, and is more general than the TCC approach, which is a special case of TCICC.

The explicit form of equations (12) in terms of CI coefficients and Hamiltonian parameters, obtained using the definition (9) for $T$, are provided for the $l \leq 4$ excitation coefficients in section VI To derive those equations, instead of the standard approach of CC that use a special notation to express particle-hole normal-ordered operators, we have used a representation of $H$ in terms of excited particles and holes operators which is described in section $\nabla$ To parametrize the $\mathrm{CI}$ coefficients, the same type of LRTD-based representation as proposed for the cluster amplitudes, and described in section VIII could be used.

\section{Choice of spin-orbital basis and reference}

When working with a single-reference expansion of the wave function such as Eq. (11), the spin-orbital basis that maximizes convergence is the natural spin-orbital basis. Since these orbitals diagonalize the single-particle density matrix $\left\langle\psi_{F C I}\left|a_{i}^{\dagger} a_{j}\right| \psi_{F C I}\right\rangle$, which does not have any time dependance, they can be computed using the source-field method described in Appendix $\mathrm{A}$ This implies that the exact wave function $\left|\psi_{F C I}\right\rangle$ is approximated by $|\psi\rangle$. For instance, one can start the calculation using Hartree-Fock spin-orbitals, then, after a first approximation of the ground state energy and wave function parameters are computed, a first approximation of the natural spin-orbitals can be computed with the sourcefield method, with which a new energy and wave function can be obtained. The procedure can then be repeated until the energy converges. If the number of spin-orbitals is $2 L$, one diagonalization of $\left\langle\psi\left|a_{i}^{\dagger} a_{j}\right| \psi\right\rangle$ requires $2 L^{2}$ different solutions of the $\mathrm{CC}$ equations for a Hamiltonian perturbed by a small source field. Although that number of different CC calculations might seem daunting, each solution is only slightly different from the unperturbed one and is therefore much faster to compute.

\section{Particle-hole representation of the Hamiltonian for single reference-based calculations}

To derive the $\mathrm{CC}$ equations, it is convenient to use a representation of the Hamiltonian $H$ where the operators are normal-ordered for both particles and holes. The standard method is to express the Hamiltonian operators using the notation $\left\{a_{i}^{\dagger} a_{j}\right\}$ and $\left\{a_{i}^{\dagger} a_{j}^{\dagger} a_{k} a_{l}\right\}$, which puts the annihilation operators for empty orbitals (in $|\phi\rangle$ ) and the creation operators for occupied orbitals at the rightmost positions $(\{\ldots\}$ is not an anticommutator in that notation $)^{4.41}$. Here, we will also use such a normal-ordered Hamiltonian, but instead of using the $\{\ldots\}$ notation, we will define excited particle and hole operators $p_{i}^{\dagger}$, for the unoccupied spin-orbitals, and $h_{i}^{\dagger}$, for the occupied spin-orbitals, respectively, and express $H$ using those operators. The resulting representation is less compact than with the $\{\ldots\}$ notation, but it accomplishes a part of the work that is otherwise required during the algebraic derivation of the equations, and thus it actually simplifies it since that part is done only once. In addition, it only uses second quantization notation and is physically intuitive, as it explicitly takes the form of a Hamiltonian acting on a vacuum and the particles and anti-particles created from it, which is how the reference $|\phi\rangle$ is treated. Finally, no distinction has to be made between empty and occupied spin-orbitals indices during the derivation with that representation because that information is included in the definition of the excited particle and hole operators.

First, let us define

$$
\begin{aligned}
t_{i j}= & \delta_{\sigma_{i} \sigma_{j}} \int d^{3} r \eta_{i}^{*}(\mathbf{r})\left(\frac{-\hbar^{2} \nabla^{2}}{2 m_{e}}+V_{e i}^{c}(\mathbf{r})\right) \eta_{j}(\mathbf{r}) \\
V_{i j k l}^{c}= & \delta_{\sigma_{i} \sigma_{l}} \delta_{\sigma_{j} \sigma_{k}} \int d^{3} r_{1} d^{3} r_{2} \eta_{i}^{*}\left(\mathbf{r}_{1}\right) \eta_{j}^{*}\left(\mathbf{r}_{2}\right) V_{e e}^{c}\left(\mathbf{r}_{1}-\mathbf{r}_{2}\right) \\
& \times \eta_{k}\left(\mathbf{r}_{2}\right) \eta_{l}\left(\mathbf{r}_{1}\right),
\end{aligned}
$$

where the indices are spin-orbital indices, $m_{e}$ is the electron mass, $\eta_{i}(\mathbf{r})$ are spatial orbitals, $\sigma_{i}=\uparrow, \downarrow$ are spin indices, $V_{e i}^{c}(\mathbf{r})$ is the static Coulomb potential experienced by an electron due to ions treated in the Born-Oppenheimer approximation, and $V_{e e}^{c}\left(\mathbf{r}_{1}-\mathbf{r}_{2}\right)$ is the electron-electron Coulomb potential. Then, the Hamiltonian in the second quantized form is

$$
\hat{H}=\sum_{i j} t_{i j} a_{i}^{\dagger} a_{j}+\frac{1}{2} \sum_{i j k l} V_{i j k l}^{c} a_{i}^{\dagger} a_{j}^{\dagger} a_{k} a_{l}
$$

or, using the anti-symmetrized Coulomb interaction $V_{i j k l}=$ $V_{i j k l}^{c}-V_{i j l k}^{c}$,

$$
\begin{aligned}
& \hat{H}=\sum_{i j} t_{i j} a_{i}^{\dagger} a_{j}+\frac{1}{4} \sum_{i j k l} V_{i j k l} a_{i}^{\dagger} a_{j}^{\dagger} a_{k} a_{l} \\
& \hat{H}=\hat{K}+\hat{V}
\end{aligned}
$$

where $\hat{K}$ is the one body term and $\hat{V}$, the two-body term. Note that, from (20) and the hermicity of the potential energy term, we have that $V_{i j k l}^{c}=V_{l k j i}^{c}$ and from the inversion symmetry of $V_{e e}^{c}\left(\mathbf{r}_{1}-\mathbf{r}_{2}\right), V_{i j k l}^{c}=V_{j i l k}^{c}$, and thus $V_{i j k l}^{c}=V_{k l i j}^{c}$. Therefore, $V_{i j k l}$ also has all those symmetries, in addition to $V_{i j k l}=-V_{i j l k}=-V_{j i k l}$.

We now define excited particle and hole operators associ- 
ated with the reference $|\phi\rangle$ :

$$
\begin{gathered}
p_{i}^{\dagger}=\left(1-n_{i}^{\phi}\right) a_{i}^{\dagger}, \\
h_{i}^{\dagger}=n_{i}^{\phi} a_{i},
\end{gathered}
$$

where $n_{i}^{\phi}=\left\langle\phi\left|a_{i}^{\dagger} a_{i}\right| \phi\right\rangle$ is the number of particles occupying spin-orbital $i$ in $|\phi\rangle$ and is thus constant. Therefore, $p_{i}^{\dagger}$ and $p_{i}$ act only on empty spin-orbitals of $|\phi\rangle$, while $h_{i}^{\dagger}$ and $h_{i}$ act only on occupied spin-orbitals of $|\phi\rangle$. From those definitions we have

$$
a_{i}^{\dagger}=p_{i}^{\dagger}+h_{i}
$$

and the anticommutation relations

$$
\begin{gathered}
\left\{p_{i}, p_{j}\right\}=0, \\
\left\{p_{i}^{\dagger}, p_{j}\right\}=\delta_{i j}\left(1-n_{i}^{\phi}\right), \\
\left\{h_{i}, h_{j}\right\}=0, \\
\left\{h_{i}^{\dagger}, h_{j}\right\}=\delta_{i j} n_{i}^{\phi}, \\
\left\{p_{i}, h_{j}\right\}=0, \\
\left\{p_{i}, h_{j}^{\dagger}\right\}=0 .
\end{gathered}
$$

Using the definitions (23), the SD (2) is written

$$
\left|\phi_{j_{1}, j_{2}, \ldots, j_{k}}^{i_{1}, i_{2}, \ldots, i_{k}}\right\rangle=p_{i_{k}}^{\dagger} h_{j_{k}}^{\dagger} p_{i_{k-1}}^{\dagger} h_{j_{k-1}}^{\dagger} \ldots p_{i_{1}}^{\dagger} h_{j_{1}}^{\dagger}|\phi\rangle .
$$

If we substitute (24) in $\hat{K}$ and put each term in the usual normal order of second quantization, i.e., with all the annihilation operators on the right, we obtain

$$
\begin{aligned}
\hat{K}=\sum_{i j} & t_{i j} p_{i}^{\dagger} p_{j}-\sum_{i j} t_{i j} h_{i}^{\dagger} h_{j}+\sum_{i j} t_{i j}\left(p_{i}^{\dagger} h_{j}^{\dagger}+\text { H.c. }\right) \\
& +\sum_{i} t_{i i} n_{i}^{\phi}
\end{aligned}
$$

where H.c. stands for Hermitian conjugate. We also have assumed that $t_{i j}$ is real and thus $t_{i j}=t_{j i}$. Here the indices can run over all spin-orbitals because of the prefactors in the definitions (23). Note how the energy of holes is formally the negative of the particles' and how the constant term $\langle\phi|\hat{K}| \phi\rangle$ appears explicitly.

Now, if we substitute (24) in $\hat{V}$, put the terms in normal order and use the symmetries of $V_{i j k l}$, we obtain

$$
\begin{aligned}
\hat{V}= & \sum_{i j k} V_{i k k j} n_{k}^{\phi} p_{i}^{\dagger} p_{j}-\sum_{i j k} V_{i k k j} n_{k}^{\phi} h_{i}^{\dagger} h_{j} \\
& +\sum_{i j k} V_{i k k j} n_{k}^{\phi}\left(p_{i}^{\dagger} h_{j}^{\dagger}+H . c .\right)+\frac{1}{2} \sum_{i j} V_{i j j i} n_{i}^{\phi} n_{j}^{\phi} \\
& +\frac{1}{4} \sum_{i j k l} V_{i j k l} p_{i}^{\dagger} p_{j}^{\dagger} p_{k} p_{l}+\frac{1}{4} \sum_{i j k l} V_{i j k l} h_{i}^{\dagger} h_{j}^{\dagger} h_{k} h_{l} \\
& +\frac{1}{4} \sum_{i j k l} V_{i j k l}\left(p_{i}^{\dagger} p_{j}^{\dagger} h_{k}^{\dagger} h_{l}^{\dagger}+H . c .\right) \\
& +\frac{1}{2} \sum_{i j k l} V_{i j k l}\left(p_{i}^{\dagger} p_{j}^{\dagger} h_{k}^{\dagger} p_{l}+\text { H.c. }\right) \\
& +\frac{1}{2} \sum_{i j k l} V_{i j k l}\left(h_{i}^{\dagger} h_{j}^{\dagger} p_{k}^{\dagger} h_{l}+\text { H.c. }\right)-\sum_{i j k l} V_{i k j l} p_{i}^{\dagger} h_{j}^{\dagger} h_{k} p_{l} .
\end{aligned}
$$

Here, it is interesting to consider only the two-body terms involving particle and hole number operators $n_{i}^{p}=p_{i}^{\dagger} p_{i}$ and $n_{i}^{h}=h_{i}^{\dagger} h_{i}$ in (30), we then obtain

$\hat{V}_{n_{p}, n_{h}}=\frac{1}{2} \sum_{i j} V_{i j j i} n_{i}^{p} n_{j}^{p}+\frac{1}{2} \sum_{i j} V_{i j j i} n_{i}^{h} n_{j}^{h}-\sum_{i j} V_{i j j i} n_{i}^{p} n_{j}^{h}$.

Therefore, assuming $V_{i j j i}>0$, holes repel each other in the same way as particles and particles and holes attract each other. From that expression and the expression for $\hat{K}$, Eq. (29), we see how particles and holes in an insulating condensed matter system and electrons and positrons in the vacuum are mathematically equivalent in the long wavelength limit.

Now, if we define

$$
t_{i j}^{\phi}=t_{i j}+\sum_{k} V_{i k k j} n_{k}^{\phi}
$$

which is called the Fock matrix, we finally obtain the particle- 
hole representation of the Hamiltonian:

$$
\begin{aligned}
\hat{H}^{\phi}= & \hat{H}-\langle\phi|\hat{H}| \phi\rangle \\
= & \hat{H}-\sum_{i} t_{i i} n_{i}^{\phi}-\frac{1}{2} \sum_{i j} V_{i j j i} n_{i}^{\phi} n_{j}^{\phi} \\
= & \sum_{i j} t_{i j}^{\phi} p_{i}^{\dagger} p_{j}-\sum_{i j} t_{i j}^{\phi} h_{i}^{\dagger} h_{j}+\sum_{i j} t_{i j}^{\phi}\left(p_{i}^{\dagger} h_{j}^{\dagger}+H . c .\right) \\
& +\frac{1}{4} \sum_{i j k l} V_{i j k l} p_{i}^{\dagger} p_{j}^{\dagger} p_{k} p_{l}+\frac{1}{4} \sum_{i j k l} V_{i j k l} h_{i}^{\dagger} h_{j}^{\dagger} h_{k} h_{l} \\
& +\frac{1}{4} \sum_{i j k l} V_{i j k l}\left(p_{i}^{\dagger} p_{j}^{\dagger} h_{k}^{\dagger} h_{l}^{\dagger}+\text { H.c. }\right) \\
& +\frac{1}{2} \sum_{i j k l} V_{i j k l}\left(p_{i}^{\dagger} p_{j}^{\dagger} h_{k}^{\dagger} p_{l}+H . c .\right) \\
& +\frac{1}{2} \sum_{i j k l} V_{i j k l}\left(h_{i}^{\dagger} h_{j}^{\dagger} p_{k}^{\dagger} h_{l}+\text { H.c. }\right)-\sum_{i j k l} V_{i k j l} p_{i}^{\dagger} h_{j}^{\dagger} h_{k} p_{l} .
\end{aligned}
$$

Although this representation is lengthier than (22), it is convenient when working with the expansion of the wave function in numbers of particle-holes excitations, Eq. (3), either in CI or CC calculations, as illustrated in Appendix B

\section{Generalized CC equations for the energy and CI wave function coefficients}

Let us now write the equations (12) for $T$ given by (9), in terms of the CI wave function coefficients and Hamiltonian parameters, and projection SD's $\left\langle\phi_{j_{1}, j_{2} \ldots j_{l}}^{i_{1} i_{2} \ldots i_{l}}\right|$ with $0 \leq l \leq 2$. To derive the equations, we have used the excited particle and hole operators, Eqs. (23), and the representation (33) of the Hamiltonian. However, although that representation is convenient for that task, it remains a quite lengthy derivation. We therefore only provide the equations here. The derivation of the equations with projection on $\langle\phi|$ and $\left\langle\phi_{j}^{i}\right|$ are given in Appendix $\mathrm{B}$, while the derivation of the equations with projection on $\left\langle\phi_{j_{1} j_{2}}^{i_{1} i_{2}}\right|$ is provided as supplemental material

Using the shifted Hamiltonian, (33), the CC equations are

$$
\begin{aligned}
\left\langle\phi\left|e^{-T} \hat{H}^{\phi} e^{T}\right| \phi\right\rangle & =\Delta E, \\
\left\langle\phi_{j_{1}, j_{2} \ldots j_{l}}^{i_{1} i_{2} \ldots i_{l}}\left|e^{-T} \hat{H}^{\phi} e^{T}\right| \phi\right\rangle & =0, \quad 1 \leq l \leq n,
\end{aligned}
$$

where $\Delta E=E-\langle\phi|\hat{H}| \phi\rangle$, and we will take $n=2$.

First, because $T$, Eq. (9), is an excitation operator, $\langle\phi| e^{-T}=\langle\phi|$. Then, taking into account the fact that $\hat{H}^{\phi}$ can destroy at most two particle-hole pairs and that $\left\langle\phi\left|\hat{H}^{\phi}\right| \phi\right\rangle=$ 0 , the equation for the energy is

$$
\begin{aligned}
\Delta E & =\left\langle\phi\left|e^{-T} \hat{H}^{\phi} e^{T}\right| \phi\right\rangle \\
& =\left\langle\phi\left|\hat{H}^{\phi}\left(1+T+\frac{1}{2} T^{2}\right)\right| \phi\right\rangle \\
\Delta E & =\left\langle\phi\left|\hat{H}^{\phi} T\right| \phi\right\rangle+\frac{1}{2}\left\langle\phi\left|\hat{H}^{\phi} T^{2}\right| \phi\right\rangle .
\end{aligned}
$$

which, in terms of CI coefficients and Hamiltonian parameters, is

$$
\Delta E=\sum_{i j} t_{i j}^{\phi} c_{j}^{i}-\frac{1}{4} \sum_{i j k l} c_{k l}^{i j} V_{i j k l}
$$

Then, the equations with projection on $\left\langle\phi_{j}^{i}\right|$ is

$$
\begin{aligned}
0 & =\left\langle\phi_{j}^{i}\left|e^{-T} \hat{H}^{\phi} e^{T}\right| \phi\right\rangle \\
& =\left\langle\phi_{j}^{i}\left|(1-T) \hat{H}^{\phi}\left(1+T+\frac{1}{2} T^{2}+\frac{1}{3 !} T^{3}\right)\right| \phi\right\rangle \\
0 & =\left\langle\phi_{j}^{i}\left|\hat{H}^{\phi}\right| \phi\right\rangle+\left\langle\phi_{j}^{i}\left|\hat{H}^{\phi} T\right| \phi\right\rangle-\left\langle\phi_{j}^{i}\left|T \hat{H}^{\phi} T\right| \phi\right\rangle+\frac{1}{2}\left\langle\phi_{j}^{i}\left|\hat{H}^{\phi} T^{2}\right| \phi\right\rangle \\
& -\frac{1}{2}\left\langle\phi_{j}^{i}\left|T \hat{H}^{\phi} T^{2}\right| \phi\right\rangle+\frac{1}{3 !}\left\langle\phi_{j}^{i}\left|\hat{H}^{\phi} T^{3}\right| \phi\right\rangle,
\end{aligned}
$$

which yields

$$
\begin{aligned}
0=t_{i j}^{\phi} & +\sum_{l} t_{i l}^{\phi} c_{j}^{l}-\sum_{l} t_{j l}^{\phi} c_{l}^{i}-\sum_{m n} V_{i m j n} c_{m}^{n} \\
& +\sum_{k l} t_{k l}^{\phi}\left(c_{j l}^{i k}-c_{j}^{i} c_{l}^{k}\right)+\frac{1}{4} \sum_{k l m} V_{k l m i} c_{j m}^{k l} \\
& -\frac{1}{4} \sum_{k l m} V_{k l m j} c_{k l}^{i m}-\frac{1}{4} \sum_{k l m n} V_{k l m n}\left(c_{m n j}^{k l i}-c_{j}^{i} c_{m n}^{k l}\right) .
\end{aligned}
$$

Finally, the equations with projection on $\left\langle\phi_{j_{1} j_{2}}^{i_{1} i_{2}}\right|$ are

$$
\begin{aligned}
0 & =\left\langle\phi_{j_{1} j_{2}}^{i_{1} i_{2}}\left|e^{-T} \hat{H}^{\phi} e^{T}\right| \phi\right\rangle \\
& =\left\langle\phi_{j_{1} j_{2}}^{i_{1} i_{2}}\left|\left(1-T+\frac{1}{2} T^{2}\right) \hat{H}^{\phi}\left(1+T+\frac{1}{2} T^{2}+\frac{1}{3 !} T^{3}+\frac{1}{4 !} T^{4}\right)\right| \phi\right\rangle \\
& =\left\langle\phi_{j_{1} j_{2}}^{i_{1} i_{2}}\left|\hat{H}^{\phi}\right| \phi\right\rangle+\left\langle\phi_{j_{1} j_{2}}^{i_{1} i_{2}}\left|\hat{H}^{\phi} T\right| \phi\right\rangle-\left\langle\phi_{j_{1} j_{2}}^{i_{1} i_{2}}\left|T \hat{H}^{\phi}\right| \phi\right\rangle+\frac{1}{2}\left\langle\phi_{j_{1} j_{2}}^{i_{1} i_{2}}\left|\hat{H}^{\phi} T^{2}\right| \phi\right\rangle-\left\langle\phi_{j_{1} j_{2}}^{i_{1} i_{2}}\left|T \hat{H}^{\phi} T\right| \phi\right\rangle+\frac{1}{3 !}\left\langle\phi_{j_{1} j_{2}}^{i_{1} i_{2}}\left|\hat{H}^{\phi} T^{3}\right| \phi\right\rangle \\
& -\frac{1}{2}\left\langle\phi_{j_{1} j_{2}}^{i_{1} i_{2}}\left|T \hat{H}^{\phi} T^{2}\right| \phi\right\rangle+\frac{1}{2}\left\langle\phi_{j_{1} j_{2}}^{i_{1} i_{2}}\left|T^{2} \hat{H}^{\phi} T\right| \phi\right\rangle+\frac{1}{4 !}\left\langle\phi_{j_{1} j_{2}}^{i_{1} i_{2}}\left|\hat{H}^{\phi} T^{4}\right| \phi\right\rangle-\frac{1}{3 !}\left\langle\phi_{j_{1} j_{2}}^{i_{1} i_{2}}\left|T \hat{H}^{\phi} T^{3}\right| \phi\right\rangle+\frac{1}{4}\left\langle\phi_{j_{1} j_{2}}^{i_{1} i_{2}}\left|T^{2} \hat{H}^{\phi} T^{2}\right| \phi\right\rangle
\end{aligned}
$$


which yields (details in supplemental material)

$$
\begin{aligned}
& 0=-V_{i_{1} i_{2} j_{1} j_{2}}+t_{i_{1} j_{1}}^{\phi} c_{j_{2}}^{i_{2}}-t_{i_{1} j_{2}}^{\phi} c_{j_{1}}^{i_{2}}-t_{i_{2} j_{1}}^{\phi} c_{j_{2}}^{i_{1}}+t_{i_{2} j_{2}}^{\phi} c_{j_{1}}^{i_{1}}-\sum_{k}\left(V_{i_{1} i_{2} j_{1} k} c_{j_{2}}^{k}-V_{i_{1} i_{2} j_{2} k} c_{j_{1}}^{k}\right) \\
& +\sum_{k}\left(V_{j_{1} j_{2} i_{1} k} c_{k}^{i_{2}}-V_{j_{1} j_{2} i_{2} k} c_{k}^{i_{1}}\right)+\sum_{k}\left(t_{i_{1} k}^{\phi} c_{j_{1} j_{2}}^{k i_{2}}+t_{i_{2} k}^{\phi} c_{j_{1} j_{2}}^{i_{1} k}\right)-\sum_{k}\left(t_{j_{1} k}^{\phi} c_{k j_{2}}^{i_{1} i_{2}}+t_{j_{2} k}^{\phi} c_{j_{1} k}^{i_{1} i_{2}}\right) \\
& -\frac{1}{2} \sum_{k l} V_{i_{1} i_{2} k l} c_{j_{1} j_{2}}^{k l}-\frac{1}{2} \sum_{k l} V_{j_{1} j_{2} k l} c_{k l}^{i_{1} i_{2}}-\sum_{k l}\left(V_{i_{1} k j_{1} l} c_{k j_{2}}^{l i_{2}}+V_{i_{2} k j_{1} l} c_{k j_{2}}^{i_{1} l}+V_{i_{1} k j_{2} l} c_{j_{1} k}^{l i_{2}}+V_{i_{2} k j_{2} l} c_{j_{1} k}^{i_{1} l}\right) \\
& +\sum_{k l} t_{k l}^{\phi} c_{l j_{1} j_{2}}^{k i_{1} i_{2}}-\frac{1}{2} \sum_{k l m}\left(V_{k l m i_{1}} c_{m j_{1} j_{2}}^{k l i_{2}}-V_{k l m i_{2}} c_{m j_{1} j_{2}}^{k l i_{1}}\right)+\frac{1}{2} \sum_{k l m}\left(V_{k l m j_{1}} c_{k l j_{2}}^{m i_{1} i_{2}}-V_{k l m j_{2}} c_{k l j_{1}}^{m i_{1} i_{2}}\right) \\
& +c_{j_{1} j_{2}}^{i_{1} i_{2}} \sum_{k l} t_{k l}^{\phi} c_{l}^{k}-\frac{1}{4} \sum_{k l m n} V_{k l m n} c_{m n j_{1} j_{2}}^{k l i_{1} i_{2}}-\frac{1}{4} c_{j_{1} j_{2}}^{i_{1} i_{2}} \sum_{k l m n} V_{k l m n} c_{m n}^{k l} \\
& +c_{j_{1} j_{2}}^{i_{1} i_{2}}\left[-\frac{1}{2}\left(\frac{t_{i_{2} j_{2}}^{\phi}}{c_{j_{2}}^{i_{2}}}+\frac{t_{i_{1} j_{2}}^{\phi}}{c_{j_{2}}^{i_{1}}}+\frac{t_{i_{2} j_{1}}^{\phi}}{c_{j_{1}}^{i_{2}}}+\frac{t_{i_{1} j_{1}}^{\phi}}{c_{j_{1}}^{i_{1}}}\right)-\frac{1}{2} \sum_{k}\left(\frac{t_{i_{2} k}^{\phi} c_{j_{2}}^{k}}{c_{j_{2}}^{i_{2}}}+\frac{t_{i_{1} k}^{\phi} c_{j_{2}}^{k}}{c_{j_{2}}^{i_{1}}}+\frac{t_{i_{2} k}^{\phi} c_{j_{1}}^{k}}{c_{j_{1}}^{i_{2}}}+\frac{t_{i_{1} k}^{\phi} c_{j_{1}}^{k}}{c_{j_{1}}^{i_{1}}}\right)\right. \\
& +\frac{1}{2} \sum_{k}\left(\frac{t_{j_{2} k}^{\phi} c_{k}^{i_{2}}}{c_{j_{2}}^{i_{2}}}+\frac{t_{j_{2} k}^{\phi} c_{k}^{i_{1}}}{c_{j_{2}}^{i_{1}}}+\frac{t_{j_{1}}^{\phi} c_{k}^{i_{2}}}{c_{j_{1}}^{i_{2}}}+\frac{t_{j_{1} k}^{\phi} c_{k}^{i_{1}}}{c_{j_{1}}^{i_{1}}}\right)+\frac{1}{2} \sum_{k l}\left(\frac{V_{i_{2} l j_{2} k} c_{l}^{k}}{c_{j_{2}}^{i_{2}}}+\frac{V_{i_{1} l j_{2} k} c_{l}^{k}}{c_{j_{2}}^{i_{1}}}+\frac{V_{i_{2} l j_{1} k} c_{l}^{k}}{c_{j_{1}}^{i_{2}}}+\frac{V_{i_{1} l j_{1} k} c_{l}^{k}}{c_{j_{1}}^{i_{1}}}\right) \\
& -\sum_{k l} t_{k l}^{\phi}\left(\frac{c_{l j_{2}}^{k i_{2}}}{c_{j_{2}}^{i_{2}}}+\frac{c_{l j_{2}}^{k i_{1}}}{c_{j_{2}}^{i_{1}}}+\frac{c_{l j_{1}}^{k i_{2}}}{c_{j_{1}}^{i_{2}}}+\frac{c_{l j_{1}}^{k i_{1}}}{c_{j_{1}}^{i_{1}}}\right)+\frac{1}{2} \sum_{k l m}\left(\frac{V_{k l m i_{2}} c_{m j_{2}}^{k l}}{c_{j_{2}}^{i_{2}}}+\frac{V_{k l m i_{1}} c_{m j_{2}}^{k l}}{c_{j_{2}}^{i_{1}}}+\frac{V_{k l m i_{2}} c_{m j_{1}}^{k l}}{c_{j_{1}}^{i_{2}}}+\frac{V_{k l m i_{1}} c_{m j_{1}}^{k l}}{c_{j_{1}}^{i_{1}}}\right) \\
& \left.-\frac{1}{2} \sum_{k l m}\left(\frac{V_{k l m j_{2}} c_{k l}^{m i_{2}}}{c_{j_{2}}^{i_{2}}}+\frac{V_{k l m j_{2}} c_{k l}^{m i_{1}}}{c_{j_{2}}^{i_{1}}}+\frac{V_{k l m j_{1}} c_{k l}^{m i_{2}}}{c_{j_{1}}^{i_{2}}}+\frac{V_{k l m j_{1}} c_{k l}^{m i_{1}}}{c_{j_{1}}^{i_{1}}}\right)+\frac{1}{8} \sum_{k l m n} V_{k l m n}\left(\frac{c_{m n n j_{2}}^{k l i_{2}}}{c_{l_{2}}^{i_{2}}}+\frac{c_{m n j_{1}}^{k l l_{2}}}{c_{j_{1}}^{i_{2}}}+\frac{c_{m n j_{2}}^{k l i_{1}}}{c_{j_{2}}^{i_{1}}}+\frac{c_{m n j_{1}}^{k l i_{1}}}{c_{j_{1}}^{i_{1}}}\right)\right] .
\end{aligned}
$$

In equations (36), (38) and (40), the upper indices in the coefficients are summed over unoccupied spin-orbitals of the reference SD $|\phi\rangle$ and the lower indices are summed over occupied spin-orbitals.

Since the form (4) with $T$ given by (9) can represent the exact wave function, Eq. (11), the equations above apply to the exact wave function. However, because they depend on the triple- and quadruple-excitation coefficients, while the number of equations is $N_{\leq 2}^{C I}$, they form an underdetermined system of equations if the coefficients have full rank. As mentioned in the previous sections, the equations can be closed by using LRTD to parametrize the coefficients in Eqs. (36), (38) and (40), such that the number of parameters grows polynomially with the number of excitations. In particular, we suggest to use the representations described in section VII that follows, which are designed to parametrize all the involved coefficients in a globally compact way.

While Eqs. (36) and (38) are very similar to CC equations, the difference with CC is clear in Eq. (40) because of the coefficients appearing as denominators. The degree of the system is therefore higher than in standard CC equations. In practice, one could either use Eq. (40) as given here, or the version without coefficients at denominators, obtained after multiplication by $c_{j_{1}}^{i_{1}} c_{j_{2}}^{i_{1}} c_{j_{1}}^{i_{2}} c_{j_{2}}^{i_{2}}$.

Note also that, for a given number of free parameters $N_{\leq n-1}^{C I}<N_{\leq n}^{T D}<N_{\leq n}^{C I}$, not all the equations with projections on $\left\langle\phi_{j_{1} j_{2} \ldots j_{n}}^{i_{1} i_{2} \ldots i_{n}}\right|$ have to be used, but only a number
$N_{n}^{C C} \geq N_{<n}^{T D}$. However, if $N_{n}^{C C}<N_{<n}^{C I}$, the projection states should be chosen carefully to take into account all the non-vanishing terms of $H$.

Finally, because Eq. (7) still applies when the operator $T$ in the CC ansatz is given by (9), the energy remains linked and is thus size-extensive, as in standard CC. Note however that Eq. (8) does not apply anymore, which is clear from the presence of coefficients at denominators in Eq. (40), which do not appear in $H e^{T}$.

\section{Tensor representations for cluster operator amplitudes or CI coefficients}

To implement the tensor extensions of CC discussed in section IV] practical LRTD-based representations are required for either the CI coefficients or the cluster operator amplitudes. In this section, we describe representations designed to be compact specifically in the context of the proposed CC extensions, which will also allow us to estimate their computational complexity. Note however that those tensor-based representations are provided to complete the discussed $\mathrm{CC}$ extensions and are not tested. Other types of polynomially bounded parametrization are possible, and the results provided in the previous sections are valid for any such parametrization.

Let us assume that we use $\mathrm{CC}$ equations without truncation of $T$ with respect to excitation number, or the generalized CC equations of section VI, where the projection SD's have two 
excitations at most, namely $n=2$ in Eq. (34). According to Eq. (40), we need low rank tensor decompositions for the $l \leq 4$ excitation coefficients to close the equations. The simplest approach would be to use generic types of LRTD such as singular value decompositions (SVD), polyadic decompositions and higher-order SVD (HOSVD) ${ }^{39}$, to decompose the sets of coefficients, or $T$-amplitudes, at each number of excitations independently. However, because we have many tensors to decompose, of orders up to eight, we can quickly loose control over the number of parameters with such an approach. On way to reduce the number of free parameters would be to use a tensor network (TN) instead. In that case however, it is not clear how to express the different sets of coefficients $\left\{c_{j_{1}, j_{2} \ldots j_{l}}^{i_{1} i_{2} \ldots i_{l}}\right\}$ using a single TN, or even how to construct the TN for a non-local Hamiltonian. In the following, we describe one possible solution to those problems, which involves multiple binary tree tensor networks (TTN) constructed from the same smaller TTN's, and then a representation of a given set of coefficients by a superposition of TTN's (STTN). This produces a globally compact structure that relies, on one hand, on the low order of the tensors, as compared to usual TN approaches, and, on the other hand, on some basic assumptions about the dominant correlations in the system, though not on the specific connections between spin-orbitals in the Hamiltonian. More specifically, the STTN primarily take into account all types of pairwise entanglement based on the charge and the spin of the excited quasiparticles involved, and only some types of entanglement between pairs and larger groups of excitations. In addition to the fact that the same tensors are shared by different decompositions, the tensors are of third order or less, so that the total number of free parameters is kept under control.

In the following examples, we will use a variant of the $c_{j_{1} j_{2} \ldots}^{i_{1} i_{2} \ldots}$ notation of Eq. (3), as if the decomposition were used to represent CI coefficients, but they can also be applied to cluster operator amplitudes. The spin indices will be labeled explicitly because different combinations of spins require different types of decompositions in order to take the Pauli exclusion principle into account.

For the single-excitation coefficients, the most compact decomposition is a truncated singular value decomposition (SVD):

$$
c_{j \downarrow}^{i \uparrow}=\sum_{k=1}^{s_{1}} \kappa_{k k 1}^{p \bar{h}} u_{i k}^{\uparrow} v_{j k}^{\downarrow}
$$

where the columns of $u^{\uparrow}$ and $v^{\downarrow}$ are orthogonal unit vectors and $s_{1} \leq \min \left(L-N_{\sigma}, N_{\sigma}\right)$. Note that the spins are opposite since the spin carried by a hole is opposite to the spin of the particle in a pair created from a single occupied spin-orbital in $|\phi\rangle$. In the absence of spin-rotational symmetry, $c_{j \uparrow}^{i \downarrow}$ is defined similarly, using the matrices $u^{\downarrow}$ and $v^{\uparrow}$ and the singular values $\kappa_{k k 1}^{\bar{p} h}$. Expression (41) is represented graphically in Fig. 1 (a).

In the following, we will label tensors using combination of $p, \bar{p}, h$ and $\bar{h}$ as superscripts, corresponding respectively to spin up and spin down particle and spin up and spin down hole. The order in which they appear will determine how they are entangled, assuming a binary tree structure, with an addi- tional coma for odd numbers of excitations, as will be seen below.

For two particle-hole excitations with opposite spins, there are three different possible decompositions based on pairwise entanglement: Singlet particle-particle and hole-hole pairing:

$$
\begin{aligned}
\left(c_{j_{1} \downarrow j_{2} \uparrow}^{i_{1} \uparrow i_{2} \downarrow}\right)_{p \bar{p} \bar{h} h}= & \sum_{k=1}^{s_{p \bar{p}}} \sum_{l=1}^{s_{h \bar{h}}} \sum_{m, n=1}^{s_{p}} \sum_{q, r=1}^{s_{h}} \lambda_{k l 1}^{p \bar{p} \bar{h} h} \kappa_{m n k}^{p \bar{p}} \kappa_{q r l}^{\bar{h} h} \\
& \times u_{i_{1} m}^{\uparrow} u_{i_{2} n}^{\downarrow} v_{j_{1} q}^{\downarrow} v_{j_{2} r}^{\uparrow}
\end{aligned}
$$

singlet particle-hole pairing:

$$
\begin{aligned}
\left(c_{j_{1} \downarrow j_{2} \uparrow}^{i_{1} \uparrow i_{2} \downarrow}\right)_{p \bar{h} \bar{p} h}= & \sum_{k, l=1}^{s_{p \bar{h}}} \sum_{m, q=1}^{s_{p}} \sum_{n, r=1}^{s_{h}} \lambda_{k l 1}^{p \bar{h} h} \kappa_{m n k}^{p \bar{h}} \kappa_{q r l}^{\bar{p} h} \\
& \times u_{i_{1} m}^{\uparrow} v_{j_{1} n}^{\downarrow} u_{i_{2} q}^{\downarrow} v_{j_{2} r}^{\uparrow},
\end{aligned}
$$

and triplet particle-hole pairing:

$$
\begin{aligned}
\left(c_{j_{1} \downarrow j_{2} \uparrow}^{i_{1} \uparrow i_{2} \downarrow}\right)_{p h \bar{p} \bar{h}}= & \sum_{k, l=1}^{s_{p h}} \sum_{m, q=1}^{s_{p}} \sum_{n, r=1}^{s_{h}} \lambda_{k l 1}^{p h \bar{p} \bar{h}} \kappa_{m n k}^{p h} \kappa_{q r l}^{\bar{p} \bar{h}} \\
& \times u_{i_{1} m}^{\uparrow} v_{j_{2} n}^{\uparrow} u_{i_{2} q}^{\downarrow} v_{j_{1} r}^{\downarrow},
\end{aligned}
$$

where the first $s_{1}$ columns of $u^{\sigma}$ and $v^{\sigma}$ and the first matrix slice of $\kappa^{p \bar{h}}$ and $\kappa^{\bar{p} h}$ are the same as in (41), and the similar decomposition for $c_{j \uparrow}^{i \downarrow}$, where $s_{1}=\min \left(s_{p}, s_{h}\right)$, and we have assumed $s_{\bar{p} h}=s_{p \bar{h}}$ and $s_{\bar{p} \bar{h}}=s_{p h}$. As shown in Appendix $\mathrm{D}$, the decompositions (42), (43) and (44) are related to combinations of SVD's by internal rotations and all become exact when the tensor dimensions are large. They are therefore not orthogonal in general. However, at small tensor dimensions they occupy different regions of Hilbert space since they are based on different composite excitations, and thus each of those decompositions allows a compact representation for its particular type of entanglement, but not for the two other types. Therefore, to obtain a compact representation allowing all those types of entanglement to coexist, we can combine (42), (43) and (44) and express the coefficients as

$$
c_{j_{1} \downarrow j_{2} \uparrow}^{i_{1} \uparrow i_{2} \downarrow}=\left(\begin{array}{c}
c_{j_{1} \downarrow j_{2} \uparrow}^{i_{1} \uparrow i_{2} \downarrow}
\end{array}\right)_{p \bar{p} \bar{h} h}+\left(c_{j_{1} \downarrow j_{2} \uparrow}^{i_{1} \uparrow i_{2} \downarrow}\right)_{p \bar{h} \bar{p} h}+\left(c_{j_{1} \downarrow j_{2} \uparrow}^{i_{1} \uparrow i_{2} \downarrow}\right)_{p h \bar{p} \bar{h}},
$$

where the dimensions of the $u, v$ and $\kappa$ tensors must be large enough to include most of the entanglement in the set of coefficients $\left\{c_{j_{1} \downarrow j_{2} \uparrow}^{i_{1} \uparrow i_{2} \downarrow}\right\}$ while the overlap between the terms must remain small to avoid redundancy. The graphical representation of Eq. (45) is shown in Fig. 1(b).

Now, let us assume $s_{p}=s_{h}=s_{1}$ so that the number of additional parameters in (45), with respect to the singleexcitation representations, depends only on $s_{p \bar{p}}, s_{h \bar{h}}, s_{p \bar{h}}$ and $s_{p h}$. In addition, let us assume that the total number of matrix slices in the $\kappa$ tensors, $S_{\kappa}=s_{p \bar{p}}+s_{h \bar{h}}+2 s_{p \bar{h}}+2 s_{p h}$, is constant, and thus the total number of parameters for the $\kappa$ tensors is constant. Then, the total number of parameters in (45) is determined only by the total numbers of elements in the $\lambda$ matrices, $N_{\lambda}=s_{p \bar{p}} s_{h \bar{h}}+s_{p \bar{h}}^{2}+s_{p h}^{2}$. The 
minimum of $N_{\lambda}$ under the constraint that $S_{\kappa}$ is constant is at $s_{p \bar{p}}=s_{h \bar{h}}=s_{p \bar{h}}=s_{p h}=S_{\kappa} / 6$. Therefore, for $s_{p}=s_{h}=s_{1}$, if the optimal representation depended only on the value of $S_{\kappa}$, the most compact representation would be the one in which all the terms have the same number of parameters in (45). In practice, the optimal values of $s_{p}$ and $s_{h}$ are different and so are the optimal $s_{p \bar{p}}, s_{h \bar{h}}, s_{p \bar{h}}$ and $s_{p h}$. It is clear however that the inclusion of different terms tends to reduce the number of parameters. In addition, including the decompositions associated with the types of correlations actually present in the set of coefficients also minimizes $S_{\kappa}$ because each type is represented in the most compact way possible. Therefore, combining the relevant decompositions in (45) tends to minimize the numbers of parameters in both the $\lambda$ and the $\kappa$ tensors and produces a very compact representation. As will become more clear below, the compactness of the STTN structure also results from the sharing of tensors amongst different decompositions.

For spin-orbital with same spin, the decompositions must respect the Pauli exclusion principle. The decompositions based on the two possible types of pairing are

$$
\begin{aligned}
& \left(c_{j_{1} \downarrow j_{2} \downarrow}^{i_{1} \uparrow i_{2} \uparrow}\right)_{p p \bar{h} \bar{h}}=\sum_{k=1}^{s_{p p}} \sum_{l=1}^{s_{h h}} \sum_{n>m=1}^{s_{p}} \sum_{r>q=1}^{s_{h}} \lambda_{k l 1}^{p p \bar{h} \bar{h}} \kappa_{m n k}^{p p} \kappa_{q r l}^{\bar{h} \bar{h}} \\
& \times\left|\begin{array}{cc}
u_{i_{1} m}^{\uparrow} & u_{i_{1} n}^{\uparrow} \\
u_{i_{2} m}^{\uparrow} & u_{i_{2} n}^{\uparrow}
\end{array}\right|\left|\begin{array}{cc}
v_{j_{1} q}^{\downarrow} & v_{j_{1} r}^{\downarrow} \\
v_{j_{2} q}^{\downarrow} & v_{j_{2} r}^{\downarrow}
\end{array}\right| \\
& \left(c_{j_{1} \downarrow j_{2} \downarrow}^{i_{1} \uparrow i_{2} \uparrow}\right)_{p \bar{h} p \bar{h}}=\sum_{k, l=1}^{s_{p \bar{h}}} \sum_{m, q=1}^{s_{p}} \sum_{n, r=1}^{s_{h}} \lambda_{k l 1}^{p \bar{h} p \bar{h}} \kappa_{m n k}^{p \bar{h}} \kappa_{q r l}^{p \bar{h}} \\
& \times u_{i_{1} m}^{\uparrow} u_{i_{2} q}^{\uparrow}\left|\begin{array}{cc}
v_{j_{1} n}^{\downarrow} & v_{j_{1} r}^{\downarrow} \\
v_{j_{2} n}^{\downarrow} & v_{j_{2} r}^{\downarrow}
\end{array}\right|
\end{aligned}
$$

where $\lambda_{k l}^{p \bar{h} p \bar{h}}$ is symmetric, and

$$
c_{j_{1} \downarrow j_{2} \downarrow}^{i_{1} \uparrow i_{2} \uparrow}=\left(c_{j_{1} \downarrow j_{2} \downarrow}^{i_{1} \uparrow i_{2} \uparrow}\right)_{p p \bar{h} \bar{h}}+\left(c_{j_{1} \downarrow j_{2} \downarrow}^{i_{1} \uparrow i_{2} \uparrow}\right)_{p \bar{h} p \bar{h}} .
$$

In the absence of spin-rotational symmetry, the similar decompositions for $c_{j_{1} \uparrow j_{2} \uparrow}^{i_{1} \downarrow i_{2} \downarrow}$ involve the different tensors $u^{\downarrow}, v^{\uparrow}$, $\kappa^{\bar{p} \bar{p}}, \kappa^{h h}, \lambda^{\bar{p} \bar{p} h h}, \kappa^{\bar{p} h}$ and $\lambda^{\bar{p} h \bar{p} h}$. In (47), the exchange of $i_{1}$ and $i_{2}$ is equivalent to exchanging the columns in the determinant, so that $\left(c_{j_{1} \downarrow j_{2} \downarrow}^{i_{1} \uparrow i_{2} \uparrow}\right)_{p \bar{h} p \bar{h}}$ is also antisymmetric with respect to those indices. Here, because $\kappa^{p \bar{h}}$ in (47) is the same as in (43), if we assumed that only $s_{p p}$ and $s_{h h}$ can be optimized in (48), the presence of the term $\left(c_{j_{1} \downarrow j_{2} \downarrow}^{i_{1} \uparrow i_{2} \uparrow}\right)_{p \bar{h} p \bar{h}}$ would allow $s_{p p}$ and $s_{h h}$ to be as small as possible. Expression (48) is depicted in Fig. 1(c), where the antisymmetrization is indicated by braquets, instead of displaying explicitly the similar decompositions corresponding to all combinations of permutations of identical particle or hole lines.

At two excitations, we can easily include all the types of
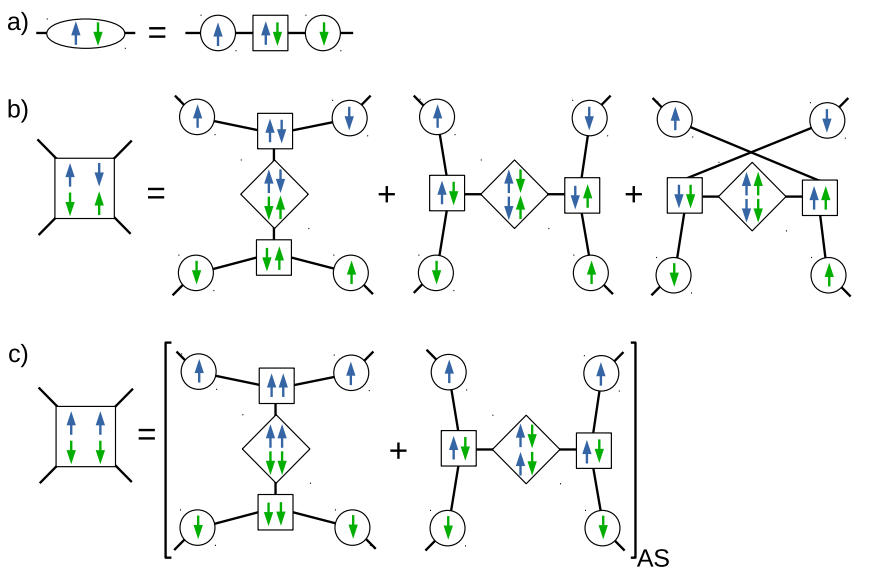

FIG. 1. Graphical representation of the STTN for single- and doubleexcitation coefficients. The blue and green arrows represent particles and holes, respectively, and their spin orientations. Figure (a) is a representation of Eq. (41), (b) corresponds to Eq. 45) and (c), Eq. (48). The circles represent the $u$ and $v$ matrices, the squares with two arrows correspond to the $\kappa$ tensors and the diamonds with four arrows, the $\lambda$ tensors. The brackets with subscript "AS" in (c) indicates that the decompositions are antisymmetrized with respect to exchange of identical particle or hole indices.

entanglement between the different pairs. In addition, all the $\kappa$ tensors connecting the pairs are also involved in the higher order decompositions described below, and are therefore required. Furthermore, if the LRTD are used to represent sets of cluster operator amplitudes, it is particularly important for the double-excitation ones to be well represented since higher order coefficients depend on them. When the number of particle-hole excitations $l$ increases, since the number of possible binary TTN increases exponentially with $l$, only an exponentially small fraction of them can be included. This is not a problem however when assuming a polynomially bounded parametrization, since it means that there necessarily exists subsets with a polynomially bounded number of decompositions that can produce accurate representations. To ensure that the strongest correlations are taken into account while keeping the number of parameters as small as possible, it is however important to include all the types of pairing in which the two quasiparticles in a pair can occupy the same spatial orbital. Those are the triplet particle-hole pairing and singlet particle-particle and hole-hole pairing. This is another reason to use superpositions of TTN instead of a single TTN representation, which is insufficient to satisfy that condition in the present context. In the following, we will keep including decompositions based on all possible types of pairing in the representations, which satisfy that condition, but also accounts for other, strictly non-local (in the space of the chosen orbital basis), correlations as well.

At three excitations, with particle-hole entanglement only 
at the lowest level of the tree, we have

$$
\begin{aligned}
& \left(c_{j_{1} \downarrow j_{2} \downarrow j_{3} \downarrow}^{i_{1} \uparrow i_{2} \uparrow i_{2} \uparrow}\right)_{p \bar{h} p \bar{h} p \bar{h}}=\sum_{m=1}^{s_{p \bar{h} p \bar{h}}} \sum_{l_{1}, l_{2}, l_{3}=1}^{s_{p \bar{h}}} \sum_{k_{1}, k_{3}, k_{5}=1}^{s_{p}} \sum_{k_{2}, k_{4}, k_{6}=1}^{s_{h}} \\
& \sum_{\pi \in S_{3}}\left(\mu_{l_{1} m}^{p \bar{h} p \bar{h} p \bar{h}} \lambda_{l_{2} l_{3} m}^{p \bar{h}}+\mu_{l_{2} m}^{p \bar{h} p \bar{h} p \bar{h}} \lambda_{l_{1} l_{3} m}^{p \bar{h}}+\mu_{l_{3} m}^{p \bar{h} p \bar{h} p \bar{h}} \lambda_{l_{1} l_{2} m}^{p \bar{h} p \bar{h}}\right) \\
& \quad \times \kappa_{k_{1} k_{2} l_{1}}^{p \bar{h}} \kappa_{k_{3} k_{4} l_{2}}^{p \bar{h}} \kappa_{k_{5} k_{6} l_{3}}^{p \bar{h}} \epsilon_{\pi_{1} \pi_{2} \pi_{3}} \\
& \quad \times u_{i_{1} k_{1}}^{\uparrow} u_{i_{2} k_{3}}^{\uparrow} u_{i_{3} k_{5}}^{\uparrow} v_{j_{\pi_{1}} k_{2}}^{\downarrow} v_{j_{\pi_{2}} k_{4}}^{\downarrow} v_{j_{\pi_{3}} k_{6}}^{\downarrow},
\end{aligned}
$$

where $\epsilon_{\pi_{1} \pi_{2} \pi_{3}}$ is the Levi-Civita symbol, $S_{3}$ is the permutation group for the set $\{1,2,3\}$, and the symmetrization of the product $\mu \lambda$ ensures the antisymmetry of the coefficient with respect to exchange of the particle (i) indices. Then, we can also have

$$
\begin{aligned}
& \left(c_{j_{1} \downarrow j_{2} \downarrow j_{3} \downarrow}^{i_{1} \uparrow i_{2} \uparrow i_{3} \uparrow}\right)_{p \bar{h}, p p \bar{h} \bar{h}}=\sum_{m=1}^{s_{p p \bar{h} \bar{h}}} \sum_{l_{1}=1}^{s_{p \bar{h}}} \sum_{l_{2}=1}^{s_{p p}} \sum_{l_{3}=1}^{s_{h h}} \\
& \sum_{k_{1}, k_{3}, k_{4}=1}^{s_{p}} \sum_{k_{2}, k_{5}, k_{6}=1}^{s_{h}} \sum_{\pi, \chi \in S_{3}} \mu_{l_{1} m}^{p \bar{h}, p p \bar{h} \bar{h}} \lambda_{l_{2} l_{3} m}^{p p \bar{h} \bar{h}} \\
& \quad \times \kappa_{k_{1} k_{2} l_{1}}^{p \bar{h}} \kappa_{k_{3} k_{4} l_{2}}^{p p} \kappa_{k_{5} k_{6} l_{3}}^{\bar{h} \bar{h}} \epsilon_{\pi_{1} \pi_{2} \pi_{3}} \epsilon_{\chi_{1} \chi_{2} \chi_{3}} \\
& \quad \times u_{i_{\pi_{1}} k_{1}}^{\uparrow} u_{i_{\pi_{2}} k_{3}}^{\uparrow} u_{i_{\pi_{1}}^{\uparrow} k_{4}}^{v_{j_{\chi_{1}}}^{\downarrow} k_{2}} v_{j_{\chi_{2}} k_{5}}^{\downarrow} v_{j_{\chi_{3}} k_{6}}^{\downarrow} .
\end{aligned}
$$

Here, we could also have entangled first the particle-hole pair with either of the two other pairs. However, to limit the number of terms we choose only one of those three possibilities, namely, the only one that respect the symmetry $(p, \bar{p}) \leftrightarrow(\bar{h}, h)$. We can then use the representation

$$
c_{j_{1} \downarrow j_{2} \downarrow j_{3} \downarrow}^{i_{1} \uparrow i_{2} \uparrow i_{3} \uparrow}=\left(c_{j_{1} \downarrow j_{2} \downarrow j_{3} \downarrow}^{i_{1} \uparrow i_{2} \uparrow i_{3} \uparrow}\right)_{p \bar{h} p \bar{h} p \bar{h}}+\left(c_{j_{1} \downarrow j_{2} \downarrow j_{3} \downarrow}^{i_{1} \uparrow i_{2} \uparrow i_{3} \uparrow}\right)_{p \bar{h}, p p \bar{h} \bar{h}},
$$

which is represented graphically in Fig. 2]a).

In the other representations described below, we will also use only decompositions that respect the $(p, \bar{p}) \leftrightarrow(\bar{h}, h)$ symmetry to simplify the representations. Otherwise, the decompositions that do not respect that symmetry would have to be included in pairs to avoid artificially breaking particle-hole symmetry. On the other hand, including only the decompositions satisfying that symmetry does not constrain the resulting wave function to be particle-hole symmetric, since that would also require that the reference and all the tensors themselves be particle-hole symmetric.

When one spin is different at three excitations, there are four combinations of pairs that respect the $(p, \bar{p}) \leftrightarrow$ $(\bar{h}, h)$ symmetry, namely, two combinations of particle-hole pairs only and two combinations with particle-hole, particleparticle and hole-hole pairs. First there is a combination of singlet particle-hole pairs:

$$
\begin{aligned}
\left(c_{j_{1} \uparrow j_{2} \downarrow j_{3} \downarrow}^{i_{1} \downarrow i_{2} \uparrow i_{3} \uparrow}\right)_{\bar{p} h, p \bar{h} p \bar{h}}= & \sum_{m=1}^{s_{p \bar{h} p \bar{h}}} \sum_{l_{1}, l_{2}, l_{3}=1}^{s_{p \bar{h}}} \sum_{k_{1}, k_{3}, k_{5}=1}^{s_{p}} \sum_{k_{2}, k_{4}, k_{6}=1}^{s_{h}} \\
& \mu_{l_{1} m}^{\bar{p} h, p \bar{h} p \bar{h}} \lambda_{l_{2} l_{3} m}^{p \bar{h} p \bar{h}} \kappa_{k_{1} k_{2} l_{1}}^{\overline{p h}} \kappa_{k_{3} k_{4} l_{2}}^{p \bar{h}} \kappa_{k_{5} k_{6} l_{3}}^{p \bar{h}} \\
& \times u_{i_{1} k_{1}}^{\downarrow} v_{j_{1} k_{2}}^{\uparrow} u_{i_{2} k_{3}}^{\uparrow} u_{i_{3} k_{5}}^{\uparrow}\left|\begin{array}{ccc}
v_{j_{2}}^{\downarrow} k_{4} & v_{j_{2} k_{6}}^{\downarrow} \\
v_{j_{3} k_{4}}^{\downarrow} & v_{j_{3} k_{6}}^{\downarrow}
\end{array}\right|,
\end{aligned}
$$

then a combination of singlet and triplet particle-hole pairs:

$$
\begin{aligned}
& \left(c_{j_{1} \uparrow j_{2} \downarrow j_{3} \downarrow}^{i_{1} \downarrow i_{2} \uparrow i_{3} \uparrow}\right)_{p \bar{h}, \bar{p} \bar{h} p h}=\sum_{m=1}^{s_{\bar{p} \bar{h} p h}} \sum_{l_{1}=1}^{s_{p \bar{h}}} \sum_{l_{2}, l_{3}=1}^{s_{p h}} \sum_{k_{1}, k_{3}, k_{5}=1}^{s_{p}} \sum_{k_{2}, k_{4}, k_{6}=1}^{s_{h}} \\
& \mu_{l_{1} m}^{p \bar{h}, \bar{p} \bar{h} p h} \lambda_{l_{2} l_{3} m}^{\bar{p} \bar{h} p h} \kappa_{k_{1} k_{2} l_{1}}^{p \bar{h}} \kappa_{k_{3} k_{4} l_{2}}^{\bar{p} \bar{h}} \kappa_{k_{5} k_{6} l_{3}}^{p h} \\
& \times u_{i_{1} k_{3}}^{\downarrow} v_{j_{1} k_{6}}^{\uparrow}\left|\begin{array}{cc}
u_{i_{2} k_{5}}^{\uparrow} & u_{i_{2} k_{1}}^{\uparrow} \\
u_{i_{3} k_{5}}^{\uparrow} & u_{i_{3} k_{1}}^{\uparrow}
\end{array}\right|\left|\begin{array}{cc}
v_{j_{2} k_{4}}^{\downarrow} & v_{j_{2} k_{2}}^{\downarrow} \\
v_{j_{3} k_{4}}^{\downarrow} & v_{j_{3} k_{2}}^{\downarrow}
\end{array}\right|
\end{aligned}
$$

a combination with triplet particle-particle and hole-hole pairs:

$$
\begin{aligned}
& \left(c_{j_{1} \uparrow j_{2} \downarrow j_{3} \downarrow}^{i_{1} \downarrow i_{2} \uparrow i_{i} \uparrow}\right)_{\bar{p} h, p p \bar{h} \bar{h}}=\sum_{m=1}^{s_{p p \bar{h} \bar{h}}} \sum_{l_{1}=1}^{s_{p \bar{h}}} \sum_{l_{2}=1}^{s_{p p}} \sum_{l_{3}=1}^{s_{h h}} \sum_{k_{1}, k_{3}, k_{4}=1}^{s_{p}} \\
& \sum_{k_{2}, k_{5}, k_{6}=1}^{s_{h}} \mu_{l_{1} m}^{\bar{p} h, p p \bar{h} \bar{h}} \lambda_{l_{2} l_{3} m}^{p p \bar{h} \bar{h}} \kappa_{k_{1} k_{2} l_{1}}^{\bar{p} h} \kappa_{k_{3} k_{4} l_{2}}^{p p} \kappa_{k_{5} k_{6} l_{3}}^{\bar{h} \bar{h}} \\
& \times u_{i_{1} k_{1}}^{\downarrow} v_{j_{1} k_{2}}^{\uparrow}\left|\begin{array}{cc}
u_{i_{2} k_{3}}^{\uparrow} & u_{i_{2} k_{4}}^{\uparrow} \\
u_{i_{3} k_{3}}^{\uparrow} & u_{i_{3} k_{4}}^{\uparrow}
\end{array}\right|\left|\begin{array}{cc}
v_{j_{2} k_{5}}^{\downarrow} & v_{j_{2} k_{6}}^{\downarrow} \\
v_{j_{3} k_{5}}^{\downarrow} & v_{j_{3} k_{6}}^{\downarrow}
\end{array}\right|
\end{aligned}
$$

and finally, a combination with singlet particle-particle and hole-hole pairs:

$$
\begin{gathered}
\left(c_{j_{1} \uparrow j_{2} \downarrow j_{3} \downarrow}^{i_{1} \downarrow i_{2} \uparrow i_{i} \uparrow}\right)_{p \bar{h}, \bar{p} p h \bar{h}}=\sum_{m=1}^{s_{\bar{p} p h \bar{h}}} \sum_{l_{1}=1}^{s_{p \bar{h}}} \sum_{l_{2}=1}^{s_{p \bar{p}}} \sum_{l_{3}=1}^{s_{h \bar{h}}} \sum_{k_{1}, k_{3}, k_{4}=1}^{s_{p}} \\
\sum_{k_{2}, k_{5}, k_{6}=1}^{s_{h}} \mu_{l_{1} m}^{p \bar{h}, \bar{p} p h \bar{h}} \lambda_{l_{2} l_{3} m}^{\bar{p} p h \bar{h}} \kappa_{k_{1} k_{2} l_{1}}^{p \bar{h}} \kappa_{k_{3} k_{4} l_{2}}^{\bar{p} p} \kappa_{k_{5} k_{6} l_{3}}^{h \bar{h}} \\
\times u_{i_{1} k_{3}}^{\downarrow} v_{j_{1} k_{5}}^{\uparrow}\left|\begin{array}{ll}
u_{i_{2} k_{4}}^{\uparrow} & u_{i_{2} k_{1}}^{\uparrow} \\
u_{i_{3} k_{4}}^{\uparrow} & u_{i_{3} k_{1}}^{\uparrow}
\end{array}\right|\left|\begin{array}{cc}
v_{j_{2} k_{6}}^{\downarrow} & v_{j_{2} k_{2}}^{\downarrow} \\
v_{j_{3} k_{6}}^{\downarrow} & v_{j_{3} k_{2}}^{\downarrow}
\end{array}\right|,
\end{gathered}
$$

where $\kappa_{k_{3} k_{4} l_{2}}^{\bar{p} p}=\kappa_{k_{4} k_{3} l_{2}}^{p \bar{p}}$ and $\kappa_{k_{5} k_{6} l_{3}}^{h \bar{h}}=\kappa_{k_{6} k_{5} l_{3}}^{\bar{h} h}$. We then use

$$
\begin{aligned}
c_{j_{1} \uparrow j_{2} \downarrow j_{3} \downarrow}^{i_{1} \downarrow i_{2} \uparrow i_{3} \uparrow}= & \left(c_{j_{1} \uparrow j_{2} \downarrow j_{3} \downarrow}^{i_{1} \downarrow i_{2} \uparrow i_{3} \uparrow}\right)_{\bar{p} h, p \bar{h} p \bar{h}}+\left(c_{j_{1} \uparrow j_{2} \downarrow j_{3} \downarrow}^{i_{1} \downarrow i_{2} \uparrow i_{3} \uparrow}\right)_{p \bar{p}, \bar{p} \bar{h} p h} \\
& +\left(c_{j_{1} \uparrow j_{2} \downarrow j_{3} \downarrow}^{i_{1} \downarrow i_{2} \uparrow}\right)_{\bar{p} h, p p \bar{h} \bar{h}}+\left(c_{j_{1} \uparrow j_{2} \downarrow j_{3} \downarrow}^{i_{1} \downarrow i_{2} \uparrow}\right)_{p \bar{h}, \bar{p} p h \bar{h}},
\end{aligned}
$$

which is depicted in Fig. 2(b).

By comparing Fig. 2 with Fig. 1, it is clear how the decompositions for the triple-excitation coefficients are con- 

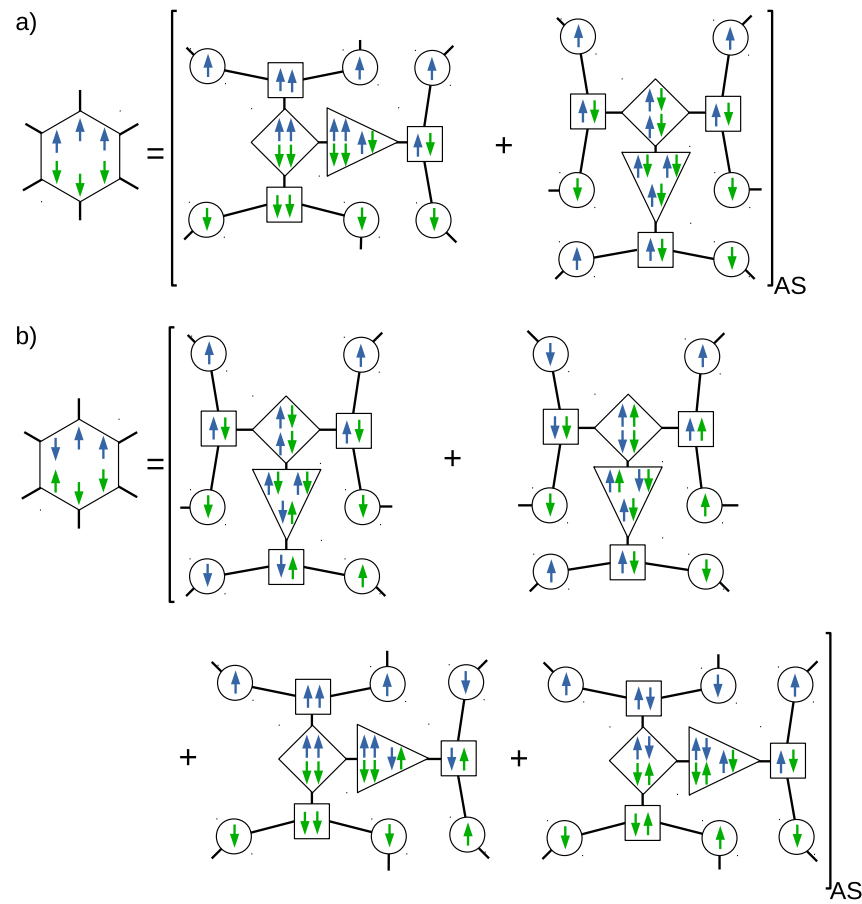

FIG. 2. Graphical representation of the STTN for triple-excitation coefficients. Figure (a) is a representation of Eq. 51 and (b) corresponds to Eq. (56). Here, the triangles represent the $\mu$ matrices. See Fig. 1 for other details on the notation. Note that, to avoid line crossings, the relative position of the circles representing the $u$ and $v$ matrices is different for different decompositions, unlike in Fig. 1 which is of no consequence since the antisymmetrization indicated by the brackets implies a summation with permutation of identical quasiparticle indices.

structed from the decompositions defining single- and doubleexcitation coefficients, using the other matrix slices of the $\lambda$ tensors. Note that other $\lambda$ tensors, not present in the representations above, are also used below in the quadruple-excitation coefficients.

For quadruple-excitation coefficients, we will only provide here the short STTN expressions and the graphical representation for the coefficients. The algebraic expressions are provided in Appendix C] As for triple-excitation coefficients, we will include decompositions with all types of pairings in the STTN representations and use only decompositions respecting the $(p, \bar{p}) \leftrightarrow(\bar{h}, h)$ symmetry.

First when all the spins are equal, we can use

$$
\begin{aligned}
& c_{j_{1} \downarrow j_{2} \downarrow j_{3} \downarrow j_{4} \downarrow}^{i_{1} \uparrow i_{2} \uparrow i_{3} \uparrow i_{4} \uparrow}=\left(c_{j_{1} \downarrow j_{2} \downarrow j_{3} \downarrow j_{4} \downarrow}^{i_{1} \uparrow i_{2} \uparrow i_{3} \uparrow i_{4} \uparrow}\right)_{p \bar{h} p \bar{h} p \bar{h} p \bar{h}}
\end{aligned}
$$

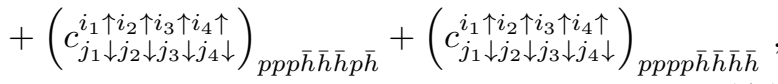
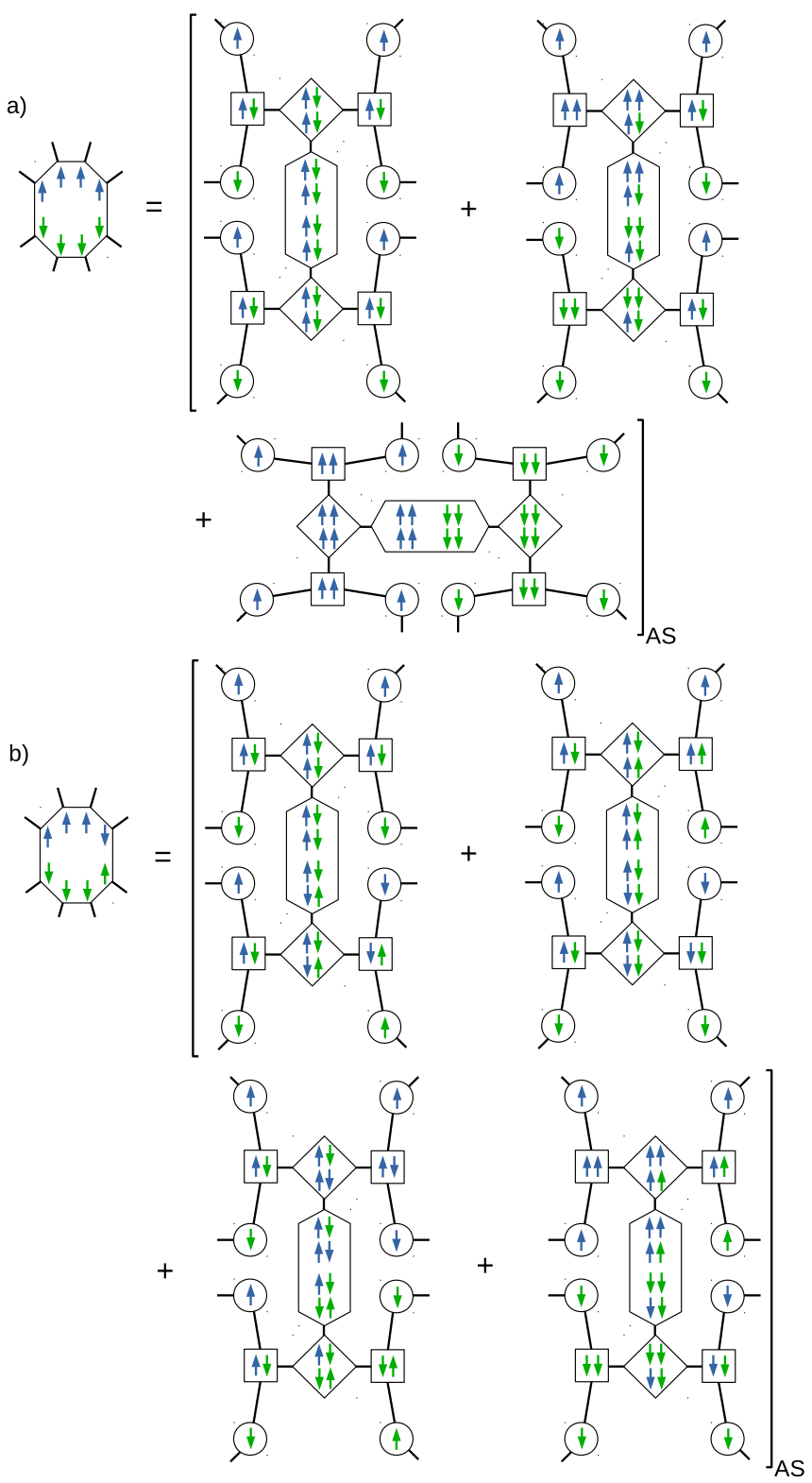

FIG. 3. Graphical representation of the quadruple-excitation coefficients (57) in (a) and 58) in (b). See Fig. 1 for details on the notation.

depicted in Fig. 3 a). Then, when one spin is different, we use

$$
\begin{aligned}
& c_{j_{1} \downarrow j_{2} \downarrow j_{3} \downarrow j_{4} \uparrow}^{i_{1} \uparrow i_{2} \uparrow i_{3} \uparrow i_{4} \downarrow}=\left(c_{j_{1} \downarrow j_{2} \downarrow j_{3} \downarrow j_{4} \uparrow}^{i_{1} \uparrow i_{2} \uparrow i_{3} \uparrow i_{i} \downarrow}\right)_{p \bar{h} p \bar{h} p \bar{h} \bar{p} h} \\
& \quad+\left(c_{j_{1} \downarrow j_{2} \downarrow j_{3} \downarrow j_{4} \uparrow}^{i_{1} \uparrow i_{2} \uparrow i_{3} \uparrow i_{4} \downarrow}\right)_{p \bar{h} p h p \bar{h} \bar{p} \bar{h}}+\left(c_{j_{1} \downarrow j_{2} \downarrow j_{3} \downarrow j_{4} \uparrow}^{i_{1} \uparrow i_{2} \uparrow i_{3} \uparrow i_{4} \downarrow}\right)_{p \bar{h} p \bar{p} p \bar{h} \bar{h} h} \\
& \quad+\left(c_{j_{1} \downarrow j_{2} \downarrow j_{3} \downarrow j_{4} \uparrow}^{i_{1} \uparrow i_{2} \uparrow i_{3} \uparrow i_{4} \downarrow}\right)_{p p p h \bar{p} \bar{h} \bar{h} \bar{h}},
\end{aligned}
$$

shown in Fig. 3 b). Finally, for two up spins and two down 


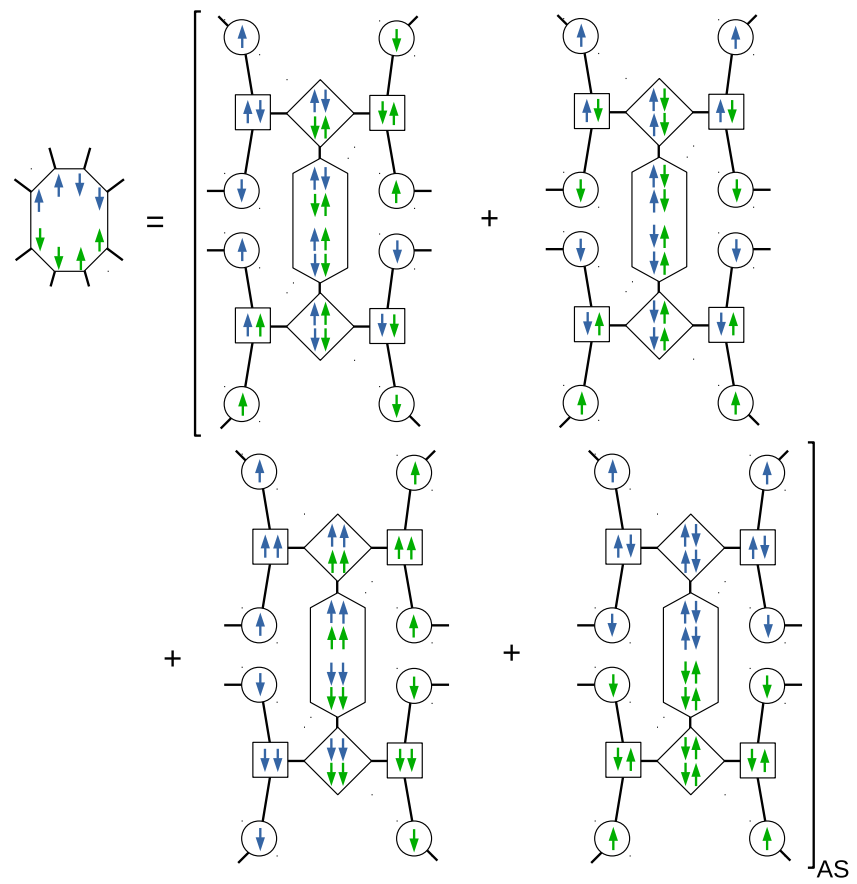

FIG. 4. Graphical representation of 59]. See Fig. 1 for details on the notation.

spins, we can use

$$
\begin{aligned}
& c_{j_{1} \downarrow j_{2} \downarrow j_{3} \uparrow j_{4} \uparrow}^{i_{1} \uparrow i_{2} \uparrow i_{3} \downarrow i_{4} \downarrow}=\left(c_{j_{1} \downarrow j_{2} \downarrow j_{3} \uparrow j_{4} \uparrow}^{i_{1} \uparrow i_{2} \uparrow i_{3} \downarrow i_{4} \downarrow}\right)_{p \bar{p} \bar{h} h p h \bar{p} \bar{h}} \\
& +\left(c_{j_{1} \downarrow j_{2} \downarrow j_{3} \uparrow j_{4} \uparrow}^{i_{1} \uparrow i_{2} \uparrow i_{3} \downarrow i_{4} \downarrow}\right)_{p \bar{h} p \bar{h} \bar{p} h \bar{p} h}+\left(c_{j_{1} \downarrow j_{2} \downarrow j_{3} \uparrow j_{4} \uparrow}^{i_{1} \uparrow i_{2} \uparrow i_{3} \downarrow i_{4} \downarrow}\right)_{p p h h \bar{p} \bar{p} \bar{h} \bar{h}} \\
& \quad+\left(c_{j_{1} \downarrow j_{2} \downarrow j_{3} \uparrow j_{4} \uparrow}^{i_{1} \uparrow i_{2} \uparrow i_{3} \downarrow i_{4} \downarrow}\right)_{p \bar{p} p \bar{p} \bar{h} h \bar{h} h}
\end{aligned}
$$

which is depicted in Fig. 4

Note that, in expressions (57), (58) and (59), depicted in Figs. 3 and 4 , the combinations of particles and holes in the two mains branches of the trees are different between different decompositions. In other words, in the representation of a given set of coefficients, say in (58) depicted in Fig. 3 (b), one cannot obtain a decomposition from another by applying permutations of quasiparticle matrices (circles) within the main branches. One instead has to apply permutations between the two main branches. The purpose of this choice is to reduce the possible overlap between the decompositions.

The above representations of coefficients are provided as examples to illustrate the STTN structure and as suggestions of representation for the sets of coefficients, or $T$-amplitudes, involved in the (generalized) $\mathrm{CC}$ equations. However, terms representing other types of entanglement can easily be added, or some included terms removed. There is in principle an optimal combination of TTN's that minimizes the total number of free parameters required to obtain an accurate representation of all the sets of coefficients. Given that the total number of free parameters is also controlled by the adjustable tensor dimensions, there is plenty of degrees of freedom for opti- mization, and any scaling with the number of particle-hole excitations can be obtained below the scaling of the number of SD's. Since the number of different sets of coefficients grows linearly with the number of excitations, if the number of terms for each set remains constant or grows only slowly, the total number of different decompositions and tensors remain bounded by low order polynomials and are thus computationally tractable, while the total number of free parameters should be reduced if the included terms are relevant.

Since the tensors always have even order, using decompositions based on pairwise entanglement is quite natural. However, from the point of view of collective excitations, this type of decomposition is based only on bosonic ones, while there can also be fermionic collective excitations in the system. For instance, the quasiparticles in a Fermi liquid are in fact collective fermionic excitations and can be approximated as single particle excitations only in an effective low-energy model. Although the representations used can also account for such collective excitations since they become exact at large tensor dimensions, to allow for a more compact representation of this kind of excitation, one could also add decompositions in which the $u$ and $v$ matrices are entangled with pairs already included. Then, for instance, those three-particles groups can be entangled with $u$ or $v$ in the double-excitation case, or together in the triple-excitation case, and so on.

The large number of adjustable tensor dimensions could be seen as a disadvantage of the STTN structure, as they requires additional optimization algorithms. However, it also offers the possibility to explore different combinations of decompositions in the STTN representation of a given set of coefficients and, assuming that the accuracy of the results can be assessed, the types of decomposition yielding the best results and the relative norms of the different terms can provide useful information about the correlations in the system.

Let us end this section by discussing the computational complexity of the proposed CC extensions, as we must also ensure that they are worth the efforts required for their implementation. The complexity in the evaluation of the generalized $\mathrm{CC}$ equations of section $\mathrm{VI}$ depends on the term

$$
\sum_{k l m n} V_{k l m n} c_{m n j_{1} j_{2}}^{k l i_{1} i_{2}}
$$

of equation (40). To simplify the complexity analysis if we use the quadruple-excitation decompositions provided in Appendix C in that term, let us set $s_{p}=s_{h}=s_{1}$, all the third dimensions of the $\kappa$ tensors to $s_{2}$ and all the third dimensions of the $\lambda$ tensors to $s_{4}$. Then, starting with the sum over the indices of $V_{k l m n}$, it requires $O\left(N^{4} s_{1}\right)$ operations, then the sum over the first two indices of the $\kappa$ tensors has $O\left(N^{4} s_{1} s_{2}^{4}\right)$ complexity, the sums over the first two indices of the $\lambda$ tensors have $O\left(N^{4} s_{2}^{4} s_{4}\right)$ or $O\left(N^{4} s_{2}^{2} s_{4}^{2}\right)$ complexity, and finally, the sum over the $\mu$ tensor indices have $O\left(N^{4} s_{4}^{2}\right)$ complexity. The overall complexity is therefore either $O\left(N^{4} s_{1} s_{2}^{4}\right)$, $O\left(N^{4} s_{2}^{4} s_{4}\right)$, or $O\left(N^{4} s_{2}^{2} s_{4}^{2}\right)$, depending on the scaling of $s_{1}$, $s_{2}$ and $s_{4}$ with $N$. The more detailed complexity analysis is provided in Appendix E For the corresponding CC equations with no truncation of $T$ with respect to the number of exci- 
tations, the limiting term has the same form, with the wave function coefficients replaced by the $T$-amplitudes, and thus the complexity is the same. The two proposed approaches are therefore tractable if the tensor dimensions are small and only weakly dependant on $N$, and their scaling can be comparable to CCSD $\left(O\left(N^{6}\right)\right)^{4}$, or better, in a certain range of tensor dimensions.

\section{Discussion}

We have seen that only low-order coefficients are relevant to the ground state energy when there exist a converging low entanglement single-reference expansion of the wave function. A remarkable aspect of that result is that it applies even if the convergence is slow, and thus the irrelevant coefficients are not vanishingly small, but only smaller than the relevant ones. This is unusual since, on one hand, most approximations based on an expansion are valid only when that expansion converges rapidly and, on the other hand, the energy of an eigenstate is usually assumed to depend on all the coefficients larger than some small threshold magnitude. In the present case, those common assumptions do not apply because of both the low entanglement property of the wave function and the locality of $H$ on the number of excitations axis. Computationally, the fact that the number of relevant parameters is much smaller than the total number of wave function parameters is an interesting advantage of using equations of the $\mathrm{CC}$ form, compared to a variational method that requires computing all the parameters. In particular, because only low-order tensors are involved, and the maximum order does not depend on system size, one can also afford tensor product representations which are not based on the specific connections between spinorbitals in the Hamiltonian, which is very convenient for nonlocal Hamiltonians. Consequently, if one suspects that there exist a single-reference expansion of the wave function that converges, even if the convergence is slow, namely at strong coupling, not only the proposed CC extensions are theoretically applicable, but they also possess important advantages compared to other tensor network methods.

If it turns out that the convergence of the CI form of the wave function is fast, then the increasing rate of the number of free parameters $N_{l}^{T D}$ with the number of excitations $l$ is necessarily slow since more components of the wave function can be neglected as $l$ increases. The low entanglement assumption is thus always valid at weak coupling. On the other hand, a slow increasing rate of $N_{l}^{T D}$ is also possible if the convergence is slow. For instance, this is the case of a strongly correlated system where many SD's are nearly degenerate in energy, hence the slow convergence of the CI coefficients with $l$, while the correlations are only local, hence the low entanglement and slow scaling of $N_{l}^{T D}$ with $l$. In fact, when the Hamiltonian is local, the low entanglement assumption is essentially always valid. It has indeed been proven recently that physically realizable ground states of such systems can only occupy an exponentially small volume of Hilbert space ${ }^{43}$, which implies that such states have low entanglement. Another exact result for local Hamiltonians is that cor- relations are short range, and thus entanglement is low, if the ground state is gapped 44 . From first principles, the Hamiltonian is not local because of the long-range Coulomb interaction. However, in metallic systems, screening effects yield effective local Hamiltonians at low energy, i.e., in the active space. Therefore, even though the original Hamiltonian is not local, based on the result of Ref. 43, it remains quite reasonable to use a low-entanglement approximation for the ground state of strongly correlated itinerant systems. However, one difficulty for such systems is to determine this effective lowenergy Hamiltonian, hence the usefulness of approaches that do not depend on the locality of the Hamiltonian. More generally, in large systems, another argument for assuming a lowentanglement ground state is that the energy range spanned by all the eigenstates grows linearly with the size of the system, while the total number of eigenstates grows exponentially. Many eigenstates therefore become nearly degenerate and can be replaced in practice by a single effective average eigenstate. Since averaging reduces correlations, this effective eigenstate has only low-entanglement. In systems in which this near degeneracy is present at the ground state energy, the low entanglement assumption is therefore valid. On the other hand, in systems with a gapped ground state that breaks the symmetry of the Hamiltonian, whether local or not, the correlation length associated with the order parameter is finite, and thus entanglement is finite as well. In summary, many different types of system have a low-entanglement ground state and can be modelled using tensor networks.

The first tensor extension of the $\mathrm{CC}$ method discussed in section IV A TCC, in which the LRTD are used to represents sets of amplitudes in the cluster operator $T$, is necessarily valid in the weak coupling limit since it includes the CC method as a special case. However, because $T$ is not truncated with respect to particle-hole excitation, and there are irreducible low-rank corrections to the reducible parts of the wave function coefficients at all orders, TCC remains theoretically valid as long as those corrections can be well represented using LRTD, as the coupling strength increases. As discussed in section VII not only the local correlations can be accounted for by the STTN representation, but also non-local ones. On the other hand, in the CC method, including its tensor implementations, the wave function coefficients at higher order than the truncation order are always completely expressed as a sum of decoupled terms, a form badly suited for strong correlations, while at the same time the importance of higher order terms increases with the coupling strength. The catastrophic failure of CC at strong coupling ${ }^{6}$ is thus inevitable unless the nature of approximation is modified. The TCC approach is a way to do so using LRTD, without projection or correlation operator. Between the two proposed approaches, TCC is the closest one to the $\mathrm{CC}$ approach, which should make it the easiest to implement.

The second proposed tensor extension of the CC method, TCICC, is in principle valid in any situation where the wave function has a converging low-entanglement CI expansion. That includes both the weak and strong coupling regimes. In practice however, at weak coupling, the parametrization would have to essentially reproduce the standard CC approx- 
imation, requiring a rather complex parametrization from the point of view of wave function coefficients instead of cluster operator amplitudes, and thus TCC is better suited in that regime. On the other hand, as discussed in section IVB at some large coupling strength, TCICC could become more compact than TCC, and thus better suited to even stronger couplings. There are also well-known strongly correlated wave functions that have a tensor network representation of the coefficients and are good variational ground states of strongly correlated systems ${ }^{16,19,23}$, suggesting that the direct parametrization of wave function coefficients in TCICC is well suited to that regime. Finally, although TCICC can also be seen as a low-entanglement version of the CI method, since the equations (36), (38) and (40) involve the CI coefficients, it is in fact very different from CI: First, Hilbert space is not truncated in the number of particle-hole excitations, then, the coefficients are not computed variationally or by matrix diagonalization, but instead the tensors defining the coefficients are obtained by solving non-linear equations, and finally, the result is size-extensive because the energy is linked.

The implementation algorithms and numerical testing of the proposed tensor extensions of the $\mathrm{CC}$ method require much more work and are thus not included here. However, at the end of section VII the obtained scaling of the calculation with system size and tensor dimensions indicate that those $\mathrm{CC}$ extensions are applicable in practice. In addition, their complexity could possibly be further improved using a tensorproduct representation for the two-particle Coulomb integrals as well24-31,45.

\section{Conclusion}

We have identified a class of fermionic wave functions which ground state energy can be computed in polynomial time using generalized $\mathrm{CC}$ equations. It corresponds to the subclass of the wave functions possessing a converging CI expansion that also possess a low-entanglement representation in which the number of free parameters $N_{<k}$ defining the wave function coefficients with $l \leq k$ particle-hole excitations is bounded polynomially in $k$. The $\mathrm{CC}$ approximation is the simplest approximation of the class, for which $N_{\leq k}$ is bounded by a constant. The convergence condition only ensures that the energy obtained from the equations is a good approximation to the true ground state energy. There is therefore no lower bound on the convergence rate, which implies that the class contains wave functions of strongly correlated systems which cannot be treated with the standard $\mathrm{CC}$ approximations. Based on that result, we have proposed extensions of the CC method to treat such systems using two types of polynomially bounded parametrization different from standard CC, and based on low-rank tensor decompositions (LRTD): a straightforward extension in which the LRTD are used to represent sets of cluster operator $(T)$ amplitudes, which involves tensor-adapted standard CC equations, and an extension of CC in which the LRTD are used to represent the CI wave function coefficients directly. For the latter case, we have derived exact generalized CC equations involving the CI coefficients with up to four particle-hole excitations. Finally, although the discussed CC extensions are in principle applicable with any type of polynomially bounded parametrization of the CI coefficients or $T$-amplitudes, to complete the proposals, we have constructed representations of the CI coefficients or cluster operator amplitudes in the form of superpositions of tree tensor networks (STTN), which by design can parametrize all the involved sets of coefficients or $T$-amplitudes in a globally compact way. If the tensor dimensions in the STTN are small and only weakly dependant on system size, the proposed CC extensions are computationally tractable.

\section{Acknowledgements}

Thanks to André-Marie Tremblay for useful discussions, comments on the manuscript, and moral support. Thanks to Thomas Baker for comments on the manuscript.

\section{A. Equal-time correlation functions}

In this appendix, we describe how to compute equal-time correlation functions using the source-field method. The derivation also corresponds to a proof of the HellmannFeynman theorem.

If the equations on the second line of Eqs. (6), are satisfied, we have

$$
\begin{aligned}
\frac{\left\langle\phi\left|e^{T^{\dagger}} \hat{H}^{\phi} e^{T}\right| \phi\right\rangle}{\left\langle\phi\left|e^{T^{\dagger}} e^{T}\right| \phi\right\rangle} & =\frac{\left\langle\phi\left|e^{T^{\dagger}} e^{T} e^{-T} \hat{H}^{\phi} e^{T}\right| \phi\right\rangle}{\left\langle\phi\left|e^{T^{\dagger}} e^{T}\right| \phi\right\rangle} \\
& =\sum_{i} \frac{\left\langle\phi\left|e^{T^{\dagger}} e^{T}\right| \phi_{i}\right\rangle\left\langle\phi_{i}\left|e^{-T} \hat{H}^{\phi} e^{T}\right| \phi\right\rangle}{\left\langle\phi\left|e^{T^{\dagger}} e^{T}\right| \phi\right\rangle} \\
& =\frac{\left\langle\phi\left|e^{T^{\dagger}} e^{T}\right| \phi\right\rangle\left\langle\phi\left|e^{-T} \hat{H}^{\phi} e^{T}\right| \phi\right\rangle}{\left\langle\phi\left|e^{T^{\dagger}} e^{T}\right| \phi\right\rangle} \\
& =\left\langle\phi\left|e^{-T} \hat{H}^{\phi} e^{T}\right| \phi\right\rangle \\
& =\Delta E .
\end{aligned}
$$

Now, if we have a perturbed Hamiltonian

$$
H_{f}=\hat{H}^{\phi}+f \hat{O}
$$

where $\hat{O}$ is any time-independent operator, and

$$
\begin{aligned}
\Delta E_{f} & =\frac{\left\langle\phi\left|e^{T_{f}^{\dagger}} H_{f} e^{T_{f}}\right| \phi\right\rangle}{\left\langle\phi\left|e^{T_{f}^{\dagger}} e^{T_{f}}\right| \phi\right\rangle} \\
& =\frac{\left\langle\psi_{f}\left|H_{f}\right| \psi_{f}\right\rangle}{\left\langle\psi_{f} \mid \psi_{f}\right\rangle} \\
& =\left\langle\phi\left|e^{-T_{f}} H_{f} e^{T_{f}}\right| \phi\right\rangle
\end{aligned}
$$


then,

$$
\begin{aligned}
\frac{\partial \Delta E_{f}}{\partial f} & \frac{\left\langle\psi_{f}\left|H_{f}\right| \psi_{f}\right\rangle}{\left\langle\psi_{f} \mid \psi_{f}\right\rangle^{2}} \frac{\partial\left\langle\psi_{f} \mid \psi_{f}\right\rangle}{\partial f}+\frac{1}{\left\langle\psi_{f} \mid \psi_{f}\right\rangle} \frac{\partial\left\langle\psi_{f}\left|H_{f}\right| \psi_{f}\right\rangle}{\partial f} \\
= & \frac{\Delta E_{f}}{\left\langle\psi_{f} \mid \psi_{f}\right\rangle} \frac{\partial\left\langle\psi_{f} \mid \psi_{f}\right\rangle}{\partial f}+\frac{1}{\left\langle\psi_{f} \mid \psi_{f}\right\rangle}\left(\frac{\partial}{\partial f}\left\langle\psi_{f}\right|\right) H_{f}\left|\psi_{f}\right\rangle \\
& +\frac{1}{\left\langle\psi_{f} \mid \psi_{f}\right\rangle}\left\langle\psi_{f}\left|H_{f} \frac{\partial}{\partial f}\right| \psi_{f}\right\rangle+\frac{1}{\left\langle\psi_{f} \mid \psi_{f}\right\rangle}\left\langle\psi_{f}\left|\left(\frac{\partial H_{f}}{\partial f}\right)\right| \psi_{f}\right\rangle \\
= & \frac{\Delta E_{f}}{\left\langle\psi_{f} \mid \psi_{f}\right\rangle} \frac{\partial\left\langle\psi_{f} \mid \psi_{f}\right\rangle}{\partial f}+\frac{\Delta E_{f}}{\left\langle\psi_{f} \mid \psi_{f}\right\rangle} \frac{\partial\left\langle\psi_{f} \mid \psi_{f}\right\rangle}{\partial f} \\
& +\frac{1}{\left\langle\psi_{f} \mid \psi_{f}\right\rangle}\left\langle\psi_{f}\left|\left(\frac{\partial H_{f}}{\partial f}\right)\right| \psi_{f}\right\rangle \\
= & \frac{2 \Delta E_{f}}{\left\langle\psi_{f} \mid \psi_{f}\right\rangle} \frac{\partial \psi_{f}\left|\psi_{f}\right\rangle}{\partial f}+\frac{1}{\left\langle\psi_{f} \mid \psi_{f}\right\rangle}\left\langle\psi_{f}\left|\left(\frac{\partial H_{f}}{\partial f}\right)\right| \psi_{f}\right\rangle .
\end{aligned}
$$

If $f$ is small enough, according to perturbation theory,

$$
\left|\psi_{f}\right\rangle \approx|\psi\rangle+f\left|\mu_{1}\right\rangle
$$

where $\left|\mu_{1}\right\rangle$ is orthogonal to $|\psi\rangle$, and

$$
\left\langle\psi_{f} \mid \psi_{f}\right\rangle=\langle\psi \mid \psi\rangle+f^{2}\left\langle\mu_{1} \mid \mu_{1}\right\rangle
$$

Thus,

$$
\begin{gathered}
\frac{\partial\left\langle\psi_{f} \mid \psi_{f}\right\rangle}{\partial f}=2 f\left\langle\mu_{1} \mid \mu_{1}\right\rangle, \\
\frac{\partial \Delta E_{f}}{\partial f}=f \frac{4 \Delta E_{f}}{\left\langle\psi_{f} \mid \psi_{f}\right\rangle}\left\langle\mu_{1} \mid \mu_{1}\right\rangle+\frac{1}{\left\langle\psi_{f} \mid \psi_{f}\right\rangle}\left\langle\psi_{f}\left|\left(\frac{\partial H_{f}}{\partial f}\right)\right| \psi_{f}\right\rangle, \\
\lim _{f \rightarrow 0} \frac{\partial \Delta E_{f}}{\partial f}=\lim _{f \rightarrow 0} \frac{1}{\left\langle\psi_{f} \mid \psi_{f}\right\rangle}\left\langle\psi_{f}\left|\left(\frac{\partial H_{f}}{\partial f}\right)\right| \psi_{f}\right\rangle
\end{gathered}
$$

and finally,

$$
\frac{\langle\psi|\hat{O}| \psi\rangle}{\langle\psi \mid \psi\rangle}=\lim _{f \rightarrow 0} \frac{\partial}{\partial f}\left\langle\phi\left|e^{-T_{f}} H_{f} e^{T_{f}}\right| \phi\right\rangle .
$$

We can therefore compute the expectation value of any timeindependent operator $\hat{O}$ by computing $\Delta E_{f}$ for $f=0$ and for $f$ small and obtain

$$
\frac{\langle\psi|\hat{O}| \psi\rangle}{\langle\psi \mid \psi\rangle} \approx \frac{\Delta E_{f}-\Delta E_{0}}{f} .
$$

In particular, if

$$
\begin{aligned}
\hat{O} & =a_{i}^{\dagger} a_{j} \\
& =\left(p_{i}^{\dagger}+h_{i}\right)\left(p_{j}+h_{j}^{\dagger}\right) \\
& =p_{i}^{\dagger} p_{j}+p_{i}^{\dagger} h_{j}^{\dagger}+h_{i} p_{j}+h_{i} h_{j}^{\dagger} .
\end{aligned}
$$

we can obtain the one particle density-matrix elements

$$
\frac{\left\langle\psi\left|a_{i}^{\dagger} a_{j}\right| \psi\right\rangle}{\langle\psi \mid \psi\rangle} .
$$

By diagonalizing the one-particle density matrix, we obtain the natural spin-orbitals basis, which is the basis that yields the fastest convergence of a configuration interaction series. Although each density matrix elements requires a different calculation, and the number of non-zero elements $2 L^{2}$ can be large, each calculation should converge rapidly if the parameters for the unperturbed Hamiltonian are used as the initial ones in each calculation since the perturbation is very small.

\section{B. Derivation of the generalized $\mathrm{CC}$ equations for the $\mathrm{CI}$ coefficients}

Here we provide the derivation of the equations (11) for $T$ given by Eq. (9), with projection of $e^{-T} H^{\phi} e^{T}|\phi\rangle$ on $\langle\phi|$ and $\left\langle\phi_{j}^{i}\right.$, using the excited particle and hole operators, Eqs. (23), and the representation (33) of the Hamiltonian. The derivation of the equations with projection on $\left\langle\phi_{j_{1} j_{2}}^{i_{1} i_{2}}\right|$ are provided as supplemental material

Taking into account the fact that $T$ is an excitation operator, and thus $\langle\phi| e^{-T}=\langle\phi|$, that $H$ can destroy at most two particle-hole pairs, and that $\left\langle\phi\left|\hat{H}^{\phi}\right| \phi\right\rangle=0$, the equation for the energy is

$$
\begin{aligned}
\Delta E & =\left\langle\phi\left|e^{-T} \hat{H}^{\phi} e^{T}\right| \phi\right\rangle \\
& =\left\langle\phi\left|\hat{H}^{\phi}\left(1+T+\frac{1}{2} T^{2}\right)\right| \phi\right\rangle \\
\Delta E & =\left\langle\phi\left|\hat{H}^{\phi} T\right| \phi\right\rangle+\frac{1}{2}\left\langle\phi\left|\hat{H}^{\phi} T^{2}\right| \phi\right\rangle .
\end{aligned}
$$

For $\left\langle\phi\left|\hat{H}^{\phi} T\right| \phi\right\rangle$, the only term in $H^{\phi}$, Eq. (33), that contributes is the term annihilating a single particle-hole pair:

$$
\begin{aligned}
\left\langle\phi\left|\hat{H}^{\phi} T\right| \phi\right\rangle & =\left\langle\phi\left|\left(\sum_{i j} t_{i j}^{\phi} h_{j} p_{i}\right)\left(\sum_{k l} c_{l}^{k} p_{k}^{\dagger} h_{l}^{\dagger}\right)\right| \phi\right\rangle \\
& =\sum_{i j k l} t_{i j}^{\phi} c_{l}^{k} \delta_{i k} \delta_{j l} \\
\left\langle\phi\left|\hat{H}^{\phi} T\right| \phi\right\rangle & =\sum_{i j} t_{i j}^{\phi} c_{j}^{i} .
\end{aligned}
$$

For $\left\langle\phi\left|\hat{H}^{\phi} T^{2}\right| \phi\right\rangle$, only the part of $H^{\phi}$ that annihilates two 
pairs contributes:

$$
\begin{aligned}
& \left\langle\phi\left|\hat{H}^{\phi} T^{2}\right| \phi\right\rangle \\
& =\frac{1}{4}\left\langle\phi\left|\left(\sum_{i j k l} V_{i j k l} h_{l} h_{k} p_{j} p_{i}\right) \frac{1}{2} \sum_{m n q s} c_{n s}^{m q} p_{m}^{\dagger} h_{n}^{\dagger} p_{q}^{\dagger} h_{s}^{\dagger}\right| \phi\right\rangle \\
& =-\frac{1}{8} \sum_{i j k l m n q s} V_{i j k l} c_{n s}^{m q}\left\langle\phi\left|p_{j} p_{i} p_{m}^{\dagger} p_{q}^{\dagger} h_{l} h_{k} h_{n}^{\dagger} h_{s}^{\dagger}\right| \phi\right\rangle \\
& =-\frac{1}{8} \sum_{i j k l m n q s} V_{i j k l} c_{n s}^{m q}\left(\delta_{i m} \delta_{j q}-\delta_{i q} \delta_{j m}\right)\left(\delta_{k n} \delta_{l s}-\delta_{k s} \delta_{l n}\right) \\
& =-\frac{1}{2} \sum_{i j k l} V_{i j k l} c_{k l}^{i j}
\end{aligned}
$$

where we have used the result (10) for $T^{2}|\phi\rangle$ and where the antisymmetry of $c_{k l}^{i j}$ or $V_{i j k l}$ can be used to obtain the last line. We thus obtain

$$
\Delta E=\sum_{i j} t_{i j}^{\phi} c_{j}^{i}-\frac{1}{4} \sum_{i j k l} c_{k l}^{i j} V_{i j k l}
$$

Note that, when reordering the products of operators to obtain a group of particle operators times a group of hole operators, the resulting sign can be obtained quickly by counting the number of particle operators on the right-hand side of each group consisting of an odd number of hole operators, and add those numbers. The sign is then negative if that number is odd.

The equations with projection on $\left\langle\phi_{j}^{i}\right|$ are

$$
\begin{aligned}
0 & =\left\langle\phi_{j}^{i}\left|e^{-T} \hat{H}^{\phi} e^{T}\right| \phi\right\rangle \\
& =\left\langle\phi_{j}^{i}\left|(1-T) \hat{H}^{\phi}\left(1+T+\frac{1}{2} T^{2}+\frac{1}{3 !} T^{3}\right)\right| \phi\right\rangle \\
0 & =\left\langle\phi_{j}^{i}\left|\hat{H}^{\phi}\right| \phi\right\rangle+\left\langle\phi_{j}^{i}\left|\hat{H}^{\phi} T\right| \phi\right\rangle-\left\langle\phi_{j}^{i}\left|T \hat{H}^{\phi} T\right| \phi\right\rangle \\
& +\frac{1}{2}\left\langle\phi_{j}^{i}\left|\hat{H}^{\phi} T^{2}\right| \phi\right\rangle-\frac{1}{2}\left\langle\phi_{j}^{i}\left|T \hat{H}^{\phi} T^{2}\right| \phi\right\rangle+\frac{1}{3 !}\left\langle\phi_{j}^{i}\left|\hat{H}^{\phi} T^{3}\right| \phi\right\rangle,
\end{aligned}
$$

For the term $\left\langle\phi_{j}^{i}\left|\hat{H}^{\phi}\right| \phi\right\rangle$, only the part of $\hat{H}^{\phi}$ that create a single particle-hole pair contributes:

$$
\begin{aligned}
& \left\langle\phi_{j}^{i}\left|\hat{H}^{\phi}\right| \phi\right\rangle=\left\langle\phi\left|h_{j} p_{i} \sum_{k l} t_{k l}^{\phi} p_{k}^{\dagger} h_{l}^{\dagger}\right| \phi\right\rangle \\
& \left\langle\phi_{j}^{i}\left|\hat{H}^{\phi}\right| \phi\right\rangle=t_{i j}^{\phi} .
\end{aligned}
$$

The term $\left\langle\phi_{j}^{i}\left|\hat{H}^{\phi} T\right| \phi\right\rangle$ includes contribution from all the terms of $\hat{H}^{\phi}$ that act on a single particle-hole pair, without creating or destroying any pair:

$$
\begin{aligned}
& \left\langle\phi_{j}^{i}\left|\hat{H}^{\phi} T\right| \phi\right\rangle \\
& =\langle\phi| h_{j} p_{i}\left(\sum_{k l} t_{k l}^{\phi} p_{k}^{\dagger} p_{l}-\sum_{k l} t_{k l}^{\phi} h_{k}^{\dagger} h_{l}\right. \\
& \left.-\sum_{k l m n} V_{k m l n} p_{k}^{\dagger} h_{l}^{\dagger} h_{m} p_{n}\right) \sum_{q r} c_{r}^{q} p_{q}^{\dagger} h_{r}^{\dagger}|\phi\rangle \\
& =\sum_{k l q r} t_{k l}^{\phi} c_{r}^{q}\left\langle\phi\left|h_{j} p_{i} p_{k}^{\dagger} p_{l} p_{q}^{\dagger} h_{r}^{\dagger}\right| \phi\right\rangle \\
& -\sum_{k l q r} t_{k l}^{\phi} c_{r}^{q}\left\langle\phi\left|h_{j} p_{i} h_{k}^{\dagger} h_{l} p_{q}^{\dagger} h_{r}^{\dagger}\right| \phi\right\rangle \\
& -\sum_{k l m n q r} V_{k m l n} c_{r}^{q}\left\langle\phi\left|h_{j} p_{i} p_{k}^{\dagger} h_{l}^{\dagger} h_{m} p_{n} p_{q}^{\dagger} h_{r}^{\dagger}\right| \phi\right\rangle \\
& =\sum_{k l q r} t_{k l}^{\phi} c_{r}^{q}\left\langle\phi\left|p_{i} p_{k}^{\dagger} p_{l} p_{q}^{\dagger} h_{j} h_{r}^{\dagger}\right| \phi\right\rangle \\
& -\sum_{k l q r} t_{k l}^{\phi} c_{r}^{q}\left\langle\phi\left|p_{i} p_{q}^{\dagger} h_{j} h_{k}^{\dagger} h_{l} h_{r}^{\dagger}\right| \phi\right\rangle \\
& -\sum_{k l m n q r} V_{k m l n} c_{r}^{q}\left\langle\phi\left|p_{i} p_{k}^{\dagger} p_{n} p_{q}^{\dagger} h_{j} h_{l}^{\dagger} h_{m} h_{r}^{\dagger}\right| \phi\right\rangle \\
& =\sum_{k l q r} t_{k l}^{\phi} c_{r}^{q} \delta_{i k} \delta_{l q} \delta_{j r}-\sum_{k l q r} t_{k l}^{\phi} c_{r}^{q} \delta_{i q} \delta_{j k} \delta_{l r} \\
& -\sum_{k l m n q r} V_{k m l n} c_{r}^{q} \delta_{i k} \delta_{n q} \delta_{j l} \delta_{m r}
\end{aligned}
$$

and thus

$$
\left\langle\phi_{j}^{i}\left|\hat{H}^{\phi} T\right| \phi\right\rangle=\sum_{l} t_{i l}^{\phi} c_{j}^{l}-\sum_{l} t_{j l}^{\phi} c_{l}^{i}-\sum_{m n} V_{i m j n} c_{m}^{n} .
$$

Now, $\left\langle\phi_{j}^{i}\left|T \hat{H}^{\phi} T\right| \phi\right\rangle$ factorizes as $\left\langle\phi_{j}^{i}|T| \phi\right\rangle\left\langle\phi\left|\hat{H}^{\phi} T\right| \phi\right\rangle$. Thus, using the result (B2),

$$
-\left\langle\phi_{j}^{i}\left|T \hat{H}^{\phi} T\right| \phi\right\rangle=-c_{j}^{i} \sum_{k l} t_{k l}^{\phi} c_{l}^{k}
$$

In $\left\langle\phi_{j}^{i}\left|\hat{H}^{\phi} T^{2}\right| \phi\right\rangle$, all the parts of $\hat{H}^{\phi}$ destroying a single pair contribute: 


$$
\begin{aligned}
\left\langle\phi_{j}^{i}\left|\hat{H}^{\phi} T^{2}\right| \phi\right\rangle & \\
= & \left\langle\phi\left|h_{j} p_{i}\left(\sum_{k l} t_{k l}^{\phi} h_{l} p_{k}+\frac{1}{4} \sum_{k l m n} V_{k l m n} p_{n}^{\dagger} h_{m} p_{l} p_{k}+\frac{1}{4} \sum_{k l m n} V_{k l m n} h_{n}^{\dagger} p_{m} h_{l} h_{k}\right) \frac{1}{2} \sum_{q r s t} c_{r t}^{q s} p_{q}^{\dagger} h_{r}^{\dagger} p_{s}^{\dagger} h_{t}^{\dagger}\right| \phi\right\rangle \\
= & \frac{1}{2} \sum_{k l q r s t} t_{k l}^{\phi} c_{r t}^{q s}\left\langle\phi\left|h_{j} p_{i} h_{l} p_{k} p_{q}^{\dagger} h_{r}^{\dagger} p_{s}^{\dagger} h_{t}^{\dagger}\right| \phi\right\rangle+\frac{1}{8} \sum_{k l m n q r s t} V_{k l m n} c_{r t}^{q s}\left\langle\phi\left|h_{j} p_{i} p_{n}^{\dagger} h_{m} p_{l} p_{k} p_{q}^{\dagger} h_{r}^{\dagger} p_{s}^{\dagger} h_{t}^{\dagger}\right| \phi\right\rangle \\
& +\frac{1}{8} \sum_{k l m n q r s t} V_{k l m n} c_{r t}^{q s}\left\langle\phi\left|h_{j} p_{i} h_{n}^{\dagger} p_{m} h_{l} h_{k} p_{q}^{\dagger} h_{r}^{\dagger} p_{s}^{\dagger} h_{t}^{\dagger}\right| \phi\right\rangle \\
= & \frac{1}{2} \sum_{k l q r s t} t_{k l}^{\phi} c_{r t}^{q s}\left\langle\phi\left|h_{j} h_{l} h_{r}^{\dagger} h_{t}^{\dagger} p_{i} p_{k} p_{q}^{\dagger} p_{s}^{\dagger}\right| \phi\right\rangle-\frac{1}{8} \sum_{k l m n q r s t} V_{k l m n} c_{r t}^{q s}\left\langle\phi\left|h_{j} h_{m} h_{r}^{\dagger} h_{t}^{\dagger} p_{i} p_{n}^{\dagger} p_{l} p_{k} p_{q}^{\dagger} p_{s}^{\dagger}\right| \phi\right\rangle \\
& +\frac{1}{8} \sum_{k l m n q r s t} V_{k l m n} c_{r t}^{q s}\left\langle\phi\left|h_{j} h_{n}^{\dagger} h_{l} h_{k} h_{r}^{\dagger} h_{t}^{\dagger} p_{i} p_{m} p_{q}^{\dagger} p_{s}^{\dagger}\right| \phi\right\rangle \\
= & \frac{1}{2} \sum_{k l q r s t} t_{k l}^{\phi} c_{r t}^{q s}\left(\delta_{l r} \delta_{j t}-\delta_{l t} \delta_{j r}\right)\left(\delta_{k q} \delta_{i s}-\delta_{k s} \delta_{i q}\right)-\frac{1}{8} \sum_{k l m n q r s t} V_{k l m n} c_{r t}^{q s}\left(\delta_{m r} \delta_{j t}-\delta_{m t} \delta_{j r}\right) \delta_{i n}\left(\delta_{k q} \delta_{l s}-\delta_{k s} \delta_{l q}\right) \\
& +\frac{1}{8} \sum_{k l m n q r s t} V_{k l m n} c_{r t}^{q s} \delta_{j n}\left(\delta_{k r} \delta_{l t}-\delta_{k t} \delta_{l r}\right)\left(\delta_{m q} \delta_{i s}-\delta_{m s} \delta_{i q}\right)
\end{aligned}
$$

which yields, using the antisymmetry of $c_{r t}^{q s}$,

$$
\frac{1}{2}\left\langle\phi_{j}^{i}\left|\hat{H}^{\phi} T^{2}\right| \phi\right\rangle=\sum_{k l} t_{k l}^{\phi} c_{j l}^{i k}+\frac{1}{4} \sum_{k l m} V_{k l m i} c_{j m}^{k l}-\frac{1}{4} \sum_{k l m} V_{k l m j} c_{k l}^{i m}
$$

Then, $\left\langle\phi_{j}^{i}\left|T \hat{H}^{\phi} T^{2}\right| \phi\right\rangle=\left\langle\phi_{j}^{i}|T| \phi\right\rangle\left\langle\phi\left|\hat{H}^{\phi} T^{2}\right| \phi\right\rangle$. Therefore, using the result of (ㅈ3),

$$
-\frac{1}{2}\left\langle\phi_{j}^{i}\left|T \hat{H}^{\phi} T^{2}\right| \phi\right\rangle=\frac{1}{4} c_{j}^{i} \sum_{k l m n} V_{k l m n} c_{m n}^{k l} .
$$

Then, $\left\langle\phi_{j}^{i}\left|\hat{H}^{\phi} T^{3}\right| \phi\right\rangle$ involves only the part of $\hat{H}^{\phi}$ destroying two pairs:

$$
\begin{aligned}
\left\langle\phi_{j}^{i}\left|\hat{H}^{\phi} T^{3}\right| \phi\right\rangle & =\frac{1}{4(3 !)} \sum_{k l m n q r q_{1} r_{1} q_{2} r_{2}} V_{k l m n} c_{r r_{1} r_{2}}^{q q_{1} q_{2}}\left\langle\phi\left|h_{j} p_{i} h_{n} h_{m} p_{l} p_{k} p_{q}^{\dagger} h_{r}^{\dagger} p_{q_{1}}^{\dagger} h_{r_{1}}^{\dagger} p_{q_{2}}^{\dagger} h_{r_{2}}^{\dagger}\right| \phi\right\rangle \\
& =-\frac{1}{4(3 !)} \sum_{k l m n q r q_{1} r_{1} q_{2} r_{2}} V_{k l m n} c_{r r_{1} r_{2}}^{q q_{1} q_{2}}\left\langle\phi\left|h_{j} h_{n} h_{m} h_{r}^{\dagger} h_{r_{1}}^{\dagger} h_{r_{2}}^{\dagger} p_{i} p_{l} p_{k} p_{q}^{\dagger} p_{q_{1}}^{\dagger} p_{q_{2}}^{\dagger}\right| \phi\right\rangle .
\end{aligned}
$$

Here, because of the antisymmetry of $c_{s_{1} s_{2}}^{q q_{1} q_{2}}$, all $(3 !)^{2}$ terms obtained by normal-ordering the operators are identical, thus

$$
\frac{1}{3 !}\left\langle\phi_{j}^{i}\left|\hat{H}^{\phi} T^{3}\right| \phi\right\rangle=-\frac{1}{4} \sum_{k l m n} V_{k l m n} c_{m n j}^{k l i} .
$$

Therefore, Eq. (B5) becomes

$$
\begin{aligned}
0=t_{i j}^{\phi} & +\sum_{k} t_{i k}^{\phi} c_{j}^{k}-\sum_{k} t_{j k}^{\phi} c_{k}^{i}-\sum_{k l} V_{i k j l} c_{k}^{l}-c_{j}^{i} \sum_{k l} t_{k l}^{\phi} c_{l}^{k} \\
& +\sum_{k l} t_{k l}^{\phi} c_{j l}^{i k}+\frac{1}{4} \sum_{k l m} V_{k l m i} c_{j m}^{k l}-\frac{1}{4} \sum_{k l m} V_{k l m j} c_{k l}^{i m} \\
& +\frac{1}{4} c_{j}^{i} \sum_{k l m n} V_{k l m n} c_{m n}^{k l}-\frac{1}{4} \sum_{k l m n} V_{k l m n} c_{m n j}^{k l i},
\end{aligned}
$$


or, if we group disconnect terms with similar connected ones,

$$
\begin{aligned}
0=t_{i j}^{\phi} & +\sum_{k} t_{i k}^{\phi} c_{j}^{k}-\sum_{k} t_{j k}^{\phi} c_{k}^{i}-\sum_{k l} V_{i k j l} c_{k}^{l} \\
& +\sum_{k l} t_{k l}^{\phi}\left(c_{j l}^{i k}-c_{j}^{i} c_{l}^{k}\right)+\frac{1}{4} \sum_{k l m} V_{k l m i} c_{j m}^{k l} \\
& -\frac{1}{4} \sum_{k l m} V_{k l m j} c_{k l}^{i m}-\frac{1}{4} \sum_{k l m n} V_{k l m n}\left(c_{m n j}^{k l i}-c_{j}^{i} c_{m n}^{k l}\right) .
\end{aligned}
$$

\section{Tensor representation for quadruple-excitation coefficients}

See the supplemental material for the derivation of the equations with projection on $\left\langle\phi_{j_{1} j_{2}}^{\imath_{1} \imath_{2}}\right|$.

Provided here are the decompositions used in the STTN representation of the quadruple-excitation CI coefficients or cluster operator amplitudes discussed in section VII.

When all spins are equal, the decomposition for the particle-hole pairing only is

$$
\begin{aligned}
& \left(c_{j_{1} \downarrow j_{2} \downarrow j_{3} \downarrow j_{4} \downarrow}^{i_{1} \uparrow i_{2} \uparrow i_{3} \uparrow i_{4} \uparrow}\right)_{p \bar{h} p \bar{h} p \bar{h} p \bar{h}}=\sum_{m_{1}, m_{2}=1}^{s_{p \bar{h} p \bar{h}}} \sum_{l_{1}, l_{2}, l_{3}, l_{4}=1}^{s_{p \bar{h}}} \\
& \sum_{k_{1}, k_{3}, k_{5}, k_{7}=1}^{s_{p}} \sum_{k_{2}, k_{4}, k_{6}, k_{8}=1}^{s_{h}} \sum_{\pi \in S_{4}} \mu_{m_{1} m_{2}}^{p \bar{h} p \bar{h}, p \bar{h} p \bar{h}} \\
& \times\left(\lambda_{l_{1} l_{2} m_{1}}^{p \bar{h} p \bar{h}} \lambda_{l_{3} l_{4} m_{2}}^{p \bar{h} p \bar{h}}+\lambda_{l_{1} l_{3} m_{1}}^{p \bar{h}} \lambda_{l_{2} l_{4} m_{2}}^{p \bar{h} p \bar{h}}+\lambda_{l_{1} l_{4} m_{1}}^{p \bar{h} p \bar{h}} \lambda_{l_{2} l_{3} m_{2}}^{p \bar{h} p \bar{h}}\right) \\
& \times \kappa_{k_{1} k_{2} l_{1}}^{p \bar{h}} \kappa_{k_{3} k_{4} l_{2}}^{p \bar{h}} \kappa_{k_{5} k_{6} l_{3}}^{p \bar{h}} \kappa_{k_{7} k_{8} l_{4}}^{p \bar{h}} \epsilon_{\pi_{1} \pi_{2} \pi_{3} \pi_{4}} \\
& \times u_{i_{1} k_{1}}^{\uparrow} u_{i_{2} k_{3}}^{\uparrow} u_{i_{3} k_{5}}^{\uparrow} u_{i_{4} k_{7}}^{\uparrow} v_{j_{\pi_{1}} k_{2}}^{\downarrow} v_{j_{\pi_{2}} k_{4}}^{\downarrow} v_{j_{\pi_{3}} k_{6}}^{\downarrow} v_{j_{\pi_{4}} k_{8}}^{\downarrow},
\end{aligned}
$$

Then, if we combine particle-hole, particle-particle and holehole pairing, we can have

$$
\begin{aligned}
& \left(c_{j_{1} \downarrow j_{2} \downarrow j_{3} \downarrow j_{4} \downarrow}^{i_{1} \uparrow i_{2} \uparrow i_{3} \uparrow i_{4} \uparrow}\right)_{p p p \bar{h} \bar{h} \bar{h} p \bar{h}}=\sum_{m_{1}=1}^{s_{p p p \bar{h}}} \sum_{m_{2}=1}^{s_{\bar{h} \bar{h} p \bar{h}}} \sum_{l_{1}=1}^{s_{p p}} \sum_{l_{2}, l_{4}=1}^{s_{p \bar{h}}} \sum_{l_{3}=1}^{s_{h h}} \\
& \sum_{k_{1}, k_{2}, k_{3}, k_{7}=1}^{s_{p}} \sum_{k_{4}, k_{5}, k_{6}, k_{8}=1}^{s_{h}} \sum_{\pi, \chi \in S_{4}} \mu_{m_{1} m_{2}}^{p p p \bar{h}, \bar{h} \bar{h} p \bar{h}} \lambda_{l_{1} l_{2} m_{1}}^{p p p \bar{h}} \lambda_{l_{3} l_{4} m_{2}}^{\bar{h} \bar{h} p \bar{h}} \\
& \times \kappa_{k_{1} k_{2} l_{1}}^{p p} \kappa_{k_{3} k_{4} l_{2}}^{p \bar{h}} \kappa_{k_{5} k_{6} l_{3}}^{\bar{h} \bar{h}} \kappa_{k_{7} k_{8} l_{4}}^{p \bar{h}} \epsilon_{\pi_{1} \pi_{2} \pi_{3} \pi_{4}} \epsilon_{\chi_{1} \chi_{2} \chi_{3} \chi_{4}} \\
& \times u_{i_{\pi_{1}} k_{1}}^{\uparrow} u_{i_{\pi_{2}} k_{2}}^{\uparrow} u_{i_{\pi_{3}} k_{3}}^{\uparrow} u_{i_{\pi_{4}} k_{7}}^{\uparrow} v_{j_{\chi_{1}} k_{4}}^{\downarrow} v_{j_{\chi_{2}} k_{5}}^{\downarrow} v_{j_{\chi_{3}} k_{6}}^{\downarrow} v_{j_{\chi_{4}} k_{8}}^{\downarrow} \text {. }
\end{aligned}
$$

Then, for particle-particle and hole-hole pairing only, the ex- pression is

$$
\begin{aligned}
& \left(\begin{array}{c}
c_{i_{1} \uparrow i_{2} \uparrow i_{3} \uparrow i_{4} \uparrow}^{j_{1} \downarrow j_{2} \downarrow j_{3} \downarrow j_{4} \downarrow}
\end{array}\right)_{p p p p \bar{h} \bar{h} \bar{h} \bar{h}}=\sum_{m_{1}=1}^{s_{p p p p}} \sum_{m_{2}=1}^{s_{\bar{h} \bar{h} \bar{h} \bar{h}}} \sum_{l_{1}, l_{2}=1}^{s_{p p}} \sum_{l_{3}, l_{4}=1}^{s_{\bar{h} \bar{h}}} \\
& \sum_{k_{1}, k_{2}, k_{3}, k_{4}=1}^{s_{p}} \sum_{k_{5}, k_{6}, k_{7}, k_{8}=1}^{s_{h}} \sum_{\pi, \chi \in S_{4}} \mu_{m_{1} m_{2}, p p p p \bar{h} \bar{h} \bar{h}}^{p} \lambda_{l_{1} l_{2} m_{1}}^{p p p p} \lambda_{l_{3} l_{4} m_{2}}^{\bar{h} \bar{h} \bar{h} \bar{h}} \\
& \times \kappa_{k_{1} k_{2} l_{1}}^{p p} \kappa_{k_{3} k_{4} l_{2}}^{p p} \kappa_{k_{5} k_{6} l_{3}}^{\bar{h} \bar{h}} \kappa_{k_{7} k_{8} l_{4}}^{\bar{h} \bar{h}} \epsilon_{\pi_{1} \pi_{2} \pi_{3} \pi_{4}} \epsilon_{\chi_{1} \chi_{2} \chi_{3} \chi_{4}} \\
& \times u_{i_{\pi_{1}} k_{1}}^{\uparrow} u_{i_{\pi_{2}} k_{2}}^{\uparrow} u_{i_{\pi_{3}} k_{3}}^{\uparrow} u_{i_{\pi_{4}} k_{4}}^{\uparrow} v_{j_{\chi_{1}} k_{5}}^{\downarrow} v_{j_{\chi_{2}} k_{6}}^{\downarrow} v_{j_{\chi_{3}} k_{7}}^{\downarrow} v_{j_{\chi_{4}} k_{8}}^{\downarrow},
\end{aligned}
$$

and finally,

$$
\begin{aligned}
& c_{j_{1} \downarrow j_{2} \downarrow j_{3} \downarrow j_{4} \downarrow}^{i_{1} \uparrow i_{2} \uparrow i_{3} \uparrow i_{4} \uparrow}=\left(c_{j_{1} \downarrow j_{2} \downarrow j_{3} \downarrow j_{4} \downarrow}^{i_{1} \uparrow i_{2} \uparrow i_{3} \uparrow i_{4} \uparrow}\right)_{p \bar{h} p \bar{h} p \bar{h} p \bar{h}} \\
& +\left(c_{j_{1} \downarrow j_{2} \downarrow j_{3} \downarrow j_{4} \downarrow}^{i_{1} \uparrow i_{2} \uparrow i_{3} \uparrow i_{4} \uparrow}\right)_{p p p \bar{h} \bar{h} \bar{h} p \bar{h}}+\left(c_{j_{1} \downarrow j_{2} \downarrow j_{3} \downarrow j_{4} \downarrow}^{i_{1} \uparrow i_{2} \uparrow i_{3} \uparrow i_{4} \uparrow}\right)_{p p p p \bar{h} \bar{h} \bar{h} \bar{h}} .
\end{aligned}
$$

Then, when one spin is different, the decomposition based on singlet particle-hole pairing only is

$$
\begin{aligned}
& \left(c_{j_{1} \downarrow j_{2} \downarrow j_{3} \downarrow j_{4} \uparrow}^{i_{1} \uparrow i_{1} \uparrow i_{3} \uparrow i_{4} \downarrow}\right)_{p \bar{h} p \bar{h} p \bar{h} \bar{p} h}=\sum_{m_{1}=1}^{s_{p \bar{h} p \bar{h}}} \sum_{m_{2}=1}^{s_{p \bar{h} \bar{p} h}} \sum_{l_{1}, l_{2}, l_{3}, l_{4}=1}^{s_{p \bar{h}}} \\
& \sum_{k_{1}, k_{3}, k_{5}, k_{7}=1}^{s_{p}} \sum_{k_{2}, k_{4}, k_{6}, k_{8}=1}^{s_{h}} \sum_{\pi \in S_{3}} \mu_{m_{1} m_{2}}^{p \bar{h} p \bar{h}, p \bar{h} \bar{p} h} \\
& \times\left(\lambda_{l_{1} l_{2} m_{1}}^{p \bar{h} p \bar{h}} \lambda_{l_{3} l_{4} m_{2}}^{p \bar{h} \bar{p} h}+\lambda_{l_{1} l_{3} m_{1}}^{p \bar{h} p \bar{h}} \lambda_{l_{2} l_{4} m_{2}}^{p \bar{h} \bar{h}}+\lambda_{l_{2} l_{3} m_{1}}^{p \bar{h} p \bar{h}} \lambda_{l_{1} l_{4} m_{2}}^{p \bar{h} \bar{p} h}\right) \\
& \times \kappa_{k_{1} k_{2} l_{1}}^{p \bar{h}} \kappa_{k_{3} k_{4} l_{2}}^{p \bar{h}} \kappa_{k_{5} k_{6} l_{3}}^{p \bar{h}} \kappa_{k_{7} k_{8} l_{4}}^{\bar{p} h} \epsilon_{\pi_{1} \pi_{2} \pi_{3}} \\
& \times u_{i_{1} k_{1}}^{\uparrow} u_{i_{2} k_{3}}^{\uparrow} u_{i_{3} k_{5}}^{\uparrow} u_{i_{4} k_{7}}^{\downarrow} v_{j_{\pi_{1}} k_{2}}^{\downarrow} v_{j_{\pi_{2}} k_{4}}^{\downarrow} v_{j_{\pi_{3}} k_{6}}^{\downarrow} v_{j_{4} k_{8}}^{\uparrow},
\end{aligned}
$$

then, we can have both singlet and triplet particle-hole pairing:

$$
\begin{aligned}
& \left(c_{j_{1} \downarrow j_{2} \downarrow j_{3} \downarrow j_{4} \uparrow}^{i_{1} \uparrow i_{2} \uparrow i_{3} \uparrow i_{4} \downarrow}\right)_{p \bar{h} p h p \bar{h} \bar{p} \bar{h}}=\sum_{m_{1}=1}^{s_{p \bar{h} p h}} \sum_{m_{2}=1}^{s_{p \bar{h}} \bar{p} \bar{h}} \sum_{l_{1}, l_{3}=1}^{s_{p \bar{h}}} \sum_{l_{2}, l_{4}=1}^{s_{p h}} \\
& \sum_{k_{1}, k_{3}, k_{5}, k_{7}=1}^{s_{p}} \sum_{k_{2}, k_{4}, k_{6}, k_{8}=1}^{s_{h}} \sum_{\pi, \chi \in S_{3}} \mu_{m_{1} m_{2}}^{p \bar{h} p h, \bar{h} \bar{p} \bar{h}} \lambda_{l_{1} l_{2} m_{1}}^{p \bar{h} p h} \\
& \times \lambda_{l_{3} l_{4} m_{2}}^{p \bar{h} \bar{h}} \kappa_{k_{1} k_{2} l_{1}}^{p \bar{h}} \kappa_{k_{3} k_{4} l_{2}}^{p h} \kappa_{k_{5} k_{6} l_{3}}^{p \bar{h}} \kappa_{k_{7} k_{8} l_{4}}^{\bar{p} \bar{h}} \epsilon_{\pi_{1} \pi_{2} \pi_{3}} \epsilon_{\chi_{1} \chi_{2} \chi_{3}} \\
& \times u_{i_{\pi_{1}} k_{1}}^{\uparrow} u_{i_{\pi_{2}} k_{3}}^{\uparrow} u_{i_{\pi_{3}} k_{5}}^{\uparrow} u_{i_{4} k_{7}}^{\downarrow} v_{j_{\chi_{1}} k_{2}}^{\downarrow} v_{j_{\chi_{2}} k_{6}}^{\downarrow} v_{j_{\chi_{3}} k_{8}}^{\downarrow} v_{j_{4} k_{4}}^{\uparrow} \text {, }
\end{aligned}
$$


singlet particle-hole, particle-particle and hole-hole pairing:

$$
\begin{aligned}
& \left(c_{j_{1} \downarrow j_{2} \downarrow j_{3} \downarrow j_{4} \uparrow}^{i_{1} \uparrow i_{2} \uparrow i_{3} \uparrow i_{4} \downarrow}\right)_{p \bar{h} p \bar{p} p \bar{h} \bar{h} h}=\sum_{m_{1}=1}^{s_{p \bar{h} p \bar{p}}} \sum_{m_{2}=1}^{s_{p \bar{h} \bar{h} h}} \sum_{l_{1}, l_{3}=1}^{s_{p \bar{h}}} \sum_{l_{2}=1}^{s_{p \bar{p}}} \sum_{l_{4}=1}^{s_{h \bar{h}}} \\
& \sum_{k_{1}, k_{3}, k_{4}, k_{5}=1}^{s_{p}} \sum_{k_{2}, k_{6}, k_{7}, k_{8}=1}^{s_{h}} \sum_{\pi, \chi \in S_{3}} \mu_{m_{1} m_{2}}^{p \bar{h} p \bar{p}, p \bar{h} \bar{h} h} \lambda_{l_{1} l_{2} m_{1}}^{p \bar{h} p \bar{p}} \\
& \times \lambda_{l_{3} l_{4} m_{2}}^{p \bar{h} \bar{h}} \kappa_{k_{1} k_{2} l_{1}}^{p \bar{h}} \kappa_{k_{3} k_{4} l_{2}}^{p \bar{p}} \kappa_{k_{5} k_{6} l_{3}}^{p \bar{h}} \kappa_{k_{7} k_{8} l_{4}}^{\bar{h} h} \epsilon_{\pi_{1} \pi_{2} \pi_{3}} \epsilon_{\chi_{1} \chi_{2} \chi_{3}} \\
& \times u_{i_{\pi_{1}} k_{1}}^{\uparrow} u_{i_{\pi_{2}} k_{3}}^{\uparrow} u_{i_{\pi_{3}} k_{5}}^{\uparrow} u_{i_{4} k_{4}}^{\downarrow} v_{j_{\chi_{1}} k_{2}}^{\downarrow} v_{j_{\chi_{2}} k_{6}}^{\downarrow} v_{j_{\chi_{3}} k_{7}}^{\downarrow} v_{j_{4} k_{8}}^{\uparrow} \text {, }
\end{aligned}
$$

or triplet particle-hole, particle-particle and hole-hole pairing:

$$
\begin{aligned}
& \left(c_{j_{1} \downarrow j_{2} \downarrow j_{3} \downarrow j_{4} \uparrow}^{i_{1} \uparrow i_{2} \uparrow i_{3} \uparrow i_{4} \downarrow}\right)_{p p p h \bar{p} \bar{h} \bar{h} \bar{h}}=\sum_{m_{1}=1}^{s_{p p p h}} \sum_{m_{2}=1}^{s_{\bar{p} \bar{h} \bar{h} \bar{h}}} \sum_{l_{1}=1}^{s_{p p}} \sum_{l_{2}, l_{3}=1}^{s_{p h}} \sum_{l_{4}=1}^{s_{h h}} \\
& \sum_{k_{1}, k_{2}, k_{3}, k_{5}=1}^{s_{p}} \sum_{k_{4}, k_{6}, k_{7}, k_{8}=1}^{s_{h}} \sum_{\pi, \chi \in S_{3}} \mu_{m_{1} m_{2}}^{p p p h \bar{p} \bar{h} \bar{h} \bar{h}} \lambda_{l_{1} l_{2} m_{1}}^{p p p h} \\
& \times \lambda_{l_{3} l_{4} m_{2}}^{\bar{p} \bar{h} \bar{h}} \kappa_{k_{1} k_{2} l_{1}}^{p p} \kappa_{k_{3} k_{4} l_{2}}^{p h} \kappa_{k_{5} k_{6} l_{3}}^{\bar{p} \bar{h}} \kappa_{k_{7} k_{8} l_{4}}^{\bar{h} \bar{h}} \epsilon_{\pi_{1} \pi_{2} \pi_{3}} \epsilon_{\chi_{1} \chi_{2} \chi_{3}} \\
& \times u_{i_{\pi_{1}} k_{1}}^{\uparrow} u_{i_{\pi_{2}} k_{2}}^{\uparrow} u_{i_{\pi_{3}} k_{3}}^{\uparrow} u_{i_{4} k_{5}}^{\downarrow} v_{j_{\chi_{1}} k_{6}}^{\downarrow} v_{j_{\chi_{2}} k_{7}}^{\downarrow} v_{j_{\chi_{3}} k_{8}}^{\downarrow} v_{j_{4} k_{4}}^{\uparrow} \text {, }
\end{aligned}
$$

and we can use

$$
\begin{aligned}
& c_{j_{1} \downarrow j_{2} \downarrow j_{3} \downarrow j_{4} \uparrow}^{i_{1} \uparrow i_{2} \uparrow i_{3} \uparrow i_{4} \downarrow}=\left(c_{j_{1} \downarrow j_{2} \downarrow j_{3} \downarrow j_{4} \uparrow}^{i_{1} \uparrow i_{2} \uparrow i_{3} \uparrow i_{4} \downarrow}\right)_{p \bar{h} p \bar{h} p \bar{h} \bar{p} h} \\
& \quad+\left(c_{j_{1} \downarrow j_{2} \downarrow j_{3} \downarrow j_{4} \uparrow}^{i_{1} \uparrow i_{3} \uparrow i_{3} \uparrow i_{4} \downarrow}\right)_{p \bar{h} p h p \bar{h} \bar{p} \bar{h}}+\left(c_{j_{1} \downarrow j_{2} \downarrow j_{3} \downarrow j_{4} \uparrow}^{i_{1} \uparrow i_{2} \uparrow i_{3} \uparrow i_{4} \downarrow}\right)_{p \bar{h} p \bar{p} p \bar{h} \bar{h} h} \\
& \quad+\left(c_{j_{1} \downarrow j_{2} \downarrow j_{3} \downarrow j_{4} \uparrow}^{i_{1} \uparrow i_{2} \uparrow i_{3} \uparrow i_{4} \downarrow}\right)_{p p p h \bar{p} \bar{h} \bar{h} \bar{h}} .
\end{aligned}
$$

Finally, for two up spins and two down spins, we can use

$$
\begin{aligned}
& \left(c_{j_{1} \downarrow j_{2} \downarrow j_{3} \uparrow j_{4} \uparrow}^{i_{1} \uparrow i_{2} \uparrow i_{3} \downarrow i_{4} \downarrow}\right)_{p \bar{p} \bar{h} h p h \bar{p} \bar{h}}=\sum_{m_{1}=1}^{s_{p \bar{p} \bar{h} h}} \sum_{m_{2}=1}^{s_{p h \bar{p} \bar{h}}} \sum_{l_{1}=1}^{s_{p \bar{p}}} \sum_{l_{2}=1}^{s_{h \bar{h}}} \sum_{l_{3}, l_{4}=1}^{s_{p h}} \\
& \sum_{k_{1}, k_{2}, k_{5}, k_{7}=1}^{s_{p}} \sum_{k_{3}, k_{4}, k_{6}, k_{8}=1}^{s_{h}} \sum_{\pi, \rho, \tau, \chi \in S_{2}} \mu_{m_{1} m_{2}}^{p \bar{p} \bar{h} h, p h \bar{p} \bar{h}} \lambda_{l_{1} l_{2} m_{1}}^{p \bar{p} \bar{h} h} \\
& \times \lambda_{l_{3} l_{4} m_{2}}^{p h \bar{h} \bar{h}} \kappa_{k_{1} k_{2} l_{1}}^{p \bar{p}} \kappa_{k_{3} k_{4} l_{2}}^{\bar{h} h} \kappa_{k_{5} k_{6} l_{3}}^{p h} \kappa_{k_{7} k_{8} l_{4}}^{\bar{p} \bar{h}} \epsilon_{\pi_{1} \pi_{2}} \epsilon_{\rho_{1} \rho_{2}} \\
& \times \epsilon_{\tau_{1} \tau_{2}} \epsilon_{\chi_{1} \chi_{2}} u_{i_{\pi_{1}} k_{1}}^{\uparrow} u_{i_{\pi_{2}} k_{5}}^{\uparrow} u_{i_{\rho_{1}+2} k_{2}}^{\downarrow} u_{i_{\rho_{2}+2} k_{7}}^{\downarrow} \\
& \times v_{j_{\tau_{1}} k_{3}}^{\downarrow} v_{j_{\tau_{2}} k_{8}}^{\downarrow} v_{j_{\chi_{1}+2} k_{4}}^{\uparrow} v_{j_{\chi_{2}+2} k_{6}}^{\uparrow} \text {, }
\end{aligned}
$$

where $\lambda^{p \bar{p} \bar{h} h}=\lambda^{\bar{p} p h \bar{h}}$ and $\lambda_{l_{3} l_{4} m_{2}}^{p h \bar{h}}=\lambda_{l_{4} l_{3} m_{2}}^{\bar{p} \bar{h} p h}$, and then,

$$
\begin{aligned}
& \left(c_{j_{1} \downarrow j_{2} \downarrow j_{3} \uparrow j_{4} \uparrow}^{i_{1} \uparrow i_{2} \uparrow i_{3} \downarrow i_{4} \downarrow}\right)_{p \bar{h} p \bar{h} \bar{p} h \bar{p} h}=\sum_{m_{1}, m_{2}=1}^{s_{p \bar{h} p \bar{h}}} \sum_{l_{1}, l_{2}, l_{3}, l_{4}=1}^{s_{p \bar{h}}} \sum_{k_{1}, k_{3}, k_{5}, k_{7}=1}^{s_{p}} \\
& \sum_{k_{2}, k_{4}, k_{6}, k_{8}=1}^{s_{h}} \sum_{\pi, \rho, \tau, \chi \in S_{2}} \mu_{m_{1} m_{2}}^{p \bar{h} p \bar{h}, \bar{p} h \bar{p} h} \lambda_{l_{1} l_{2} m_{1}}^{p \bar{h} p \bar{h}} \lambda_{l_{3} l_{4} m_{2}}^{\bar{p} h \bar{p} h} \\
& \times \kappa_{k_{1} k_{2} l_{1}}^{p \bar{h}} \kappa_{k_{3} k_{4} l_{2}}^{p \bar{h}} \kappa_{k_{5} k_{6} l_{3}}^{\bar{p} h} \kappa_{k_{7} k_{8} l_{4}}^{\bar{p} h} \epsilon_{\pi_{1} \pi_{2}} \epsilon_{\rho_{1} \rho_{2}} \epsilon_{\tau_{1} \tau_{2}} \epsilon_{\chi_{1} \chi_{2}} \\
& \times u_{i_{\pi_{1}} k_{1}}^{\uparrow} u_{i_{\pi_{2}} k_{3}}^{\uparrow} u_{i_{\rho_{1}+2} k_{5}}^{\downarrow} u_{i_{\rho_{2}+2} k_{7}}^{\downarrow} \\
& \times v_{j_{\tau_{1}} k_{2}}^{\downarrow} v_{j_{\tau_{2}} k_{4}}^{\downarrow} v_{j_{\chi_{1}+2} k_{6}}^{\uparrow} v_{j_{\chi_{2}+2} k_{8}}^{\uparrow},
\end{aligned}
$$

$$
\begin{aligned}
& \left(c_{j_{1} \downarrow j_{2} \downarrow j_{3} \uparrow j_{4} \uparrow}^{i_{1} \uparrow i_{2} \uparrow i_{3} \downarrow i_{4} \downarrow}\right)_{p p h h \bar{p} \bar{p} \bar{h} \bar{h}}=\sum_{m_{1}, m_{2}=1}^{s_{p p h h}} \sum_{l_{1}, l_{3}=1}^{s_{p p}} \sum_{l_{3}, l_{4}=1}^{s_{h h}} \\
& \sum_{k_{1}, k_{2}, k_{5}, k_{7}=1}^{s_{p}} \sum_{k_{3}, k_{4}, k_{6}, k_{8}=1}^{s_{h}} \sum_{\pi, \rho, \tau, \chi \in S_{2}} \mu_{m_{1} m_{2}}^{p p h h, \bar{p} \bar{h} \bar{h}} \lambda_{l_{1} l_{2} m_{1}}^{p p h h} \\
& \times \lambda_{l_{3} l_{4} m_{2}}^{\bar{p} \bar{h} \bar{h}} \kappa_{k_{1} k_{2} l_{1}}^{p p} \kappa_{k_{3} k_{4} l_{2}}^{h h} \kappa_{k_{5} k_{6} l_{3}}^{\bar{p} \bar{p}} \kappa_{k_{7} k_{8} l_{4}}^{\bar{h} \bar{h}} \epsilon_{\pi_{1} \pi_{2}} \epsilon_{\rho_{1} \rho_{2}} \\
& \times \epsilon_{\tau_{1} \tau_{2}} \epsilon_{\chi_{1} \chi_{2}} u_{i_{\pi_{1}} k_{1}}^{\uparrow} u_{i_{\pi_{2}} k_{2}}^{\uparrow} u_{i_{\rho_{1}+2} k_{5}}^{\downarrow} u_{i_{\rho_{2}+2} k_{6}}^{\downarrow} \\
& \times v_{j_{\tau_{1}} k_{7}}^{\downarrow} v_{j_{\tau_{2}} k_{8}}^{\downarrow} v_{j_{\chi_{1}+2} k_{3}}^{\uparrow} v_{j_{\chi_{2}+2} k_{4}}^{\uparrow},
\end{aligned}
$$

$$
\begin{aligned}
& \left(\begin{array}{c}
c_{i_{1} \uparrow i_{2} \uparrow i_{3} \downarrow i_{4} \downarrow}^{j_{1} \downarrow j_{2} \downarrow j_{3} \uparrow j_{4} \uparrow}
\end{array}\right)_{p \bar{p} p \bar{p} \bar{h} h \bar{h} h}=\sum_{m_{1}=1}^{s_{p \bar{p} p \bar{p}}} \sum_{m_{2}=1}^{s_{\bar{h} h \bar{h} h}} \sum_{l_{1}, l_{2}=1}^{s_{p \bar{p}}} \sum_{l_{3}, l_{4}=1}^{s_{\bar{h} h}} \\
& \sum_{k_{1}, k_{2}, k_{3}, k_{4}=1}^{s_{p}} \sum_{k_{5}, k_{6}, k_{7}, k_{8}=1}^{s_{h}} \sum_{\pi, \rho, \tau, \chi \in S_{2}} \mu_{m_{1} m_{2}}^{p \bar{p} p \bar{h} h \bar{h} h} \lambda_{l_{1} l_{2} m_{1}}^{p \bar{p} p \bar{p}} \\
& \times \lambda_{l_{3} l_{4} m_{2}}^{\bar{h} h \bar{h} h} \kappa_{k_{1} k_{2} l_{1}}^{p \bar{p}} \kappa_{k_{3} k_{4} l_{2}}^{p \bar{p}} \kappa_{k_{5} k_{6} l_{3}}^{\bar{h} h} \kappa_{k_{7} k_{8} l_{4}}^{\bar{h} h} \epsilon_{\pi_{1} \pi_{2}} \epsilon_{\rho_{1} \rho_{2}} \\
& \times \epsilon_{\tau_{1} \tau_{2}} \epsilon_{\chi_{1} \chi_{2}} u_{i_{\pi_{1}} k_{1}}^{\uparrow} u_{i_{\pi_{2}} k_{3}}^{\uparrow} u_{i_{\rho_{1}+2} k_{2}}^{\downarrow} u_{i_{\rho_{2}+2} k_{4}}^{\downarrow} \\
& \times v_{j_{\tau_{1}} k_{5}}^{\downarrow} v_{j_{\tau_{2}} k_{7}}^{\downarrow} v_{j_{\chi_{1}+2} k_{6}}^{\uparrow} v_{j_{\chi_{2}+2} k_{8}}^{\uparrow} \text {, }
\end{aligned}
$$

and we use

$$
\begin{aligned}
& c_{j_{1} \downarrow j_{2} \downarrow j_{3} \uparrow j_{4} \uparrow}^{i_{1} \uparrow i_{2} \uparrow i_{3} \downarrow i_{4} \downarrow}=\left(\begin{array}{c}
c_{j_{1} \uparrow i_{2} \uparrow i_{3} \downarrow i_{2} \downarrow}^{i_{1} \downarrow j_{2} \downarrow} j_{3} \uparrow j_{4} \uparrow
\end{array}\right)_{p \bar{p} \bar{h} h p h \bar{p} \bar{h}} \\
& +\left(\begin{array}{c}
c_{i_{1} \uparrow i_{2} \uparrow i_{3} \downarrow i_{4} \downarrow}^{j_{1} \downarrow j_{2} \downarrow j_{3} \uparrow j_{4} \uparrow}
\end{array}\right)_{p \bar{h} p \bar{h} \bar{p} h \bar{p} h}+\left(\begin{array}{c}
c_{1} \uparrow i_{2} \uparrow i_{3} \downarrow i_{4} \downarrow \\
c_{1} \downarrow j_{2} \downarrow j_{3} \uparrow j_{4} \uparrow
\end{array}\right)_{p p h h \bar{p} \bar{p} \bar{h} \bar{h}} \\
& +\left(c_{j_{1} \downarrow j_{2} \downarrow j_{3} \uparrow j_{4} \uparrow}^{i_{1} \uparrow i_{2} \uparrow i_{3} \downarrow i_{4} \downarrow}\right)_{p \bar{p} p \bar{p} \bar{h} h \bar{h} h} .
\end{aligned}
$$




\section{SVD form of the tree tensor networks}

Let us see how the decomposition (42),

$$
\begin{aligned}
\left(c_{j_{1} \downarrow j_{2} \uparrow}^{i_{1} \uparrow i_{2} \downarrow}\right)_{p \bar{p} \bar{h} h}= & \sum_{k=1}^{s_{p \bar{p}}} \sum_{l=1}^{s_{h \bar{h}}} \sum_{m, n=1}^{s_{p}} \sum_{q, r=1}^{s_{h}} \lambda_{k l 1}^{p \bar{h} h} \kappa_{m n k}^{p \bar{p}} \kappa_{q r l}^{\bar{h} h} \\
& \times u_{i_{1} m}^{\uparrow} u_{i_{2} n}^{\downarrow} v_{j_{1} q}^{\downarrow} v_{j_{2} r}^{\uparrow},
\end{aligned}
$$

can be written as a combination of SVD's if the matrix slices of the $\kappa$ tensors, labeled by their third index, are orthogonal. First, we define

$$
w_{\left(i_{1} i_{2}\right), k}^{p \bar{p}}=\sum_{m, n=1}^{s_{p}} \kappa_{m n k}^{p \bar{p}} u_{i_{1} m}^{\uparrow} u_{i_{2} n}^{\downarrow}
$$

and

$$
w_{\left(j_{1}, j_{2}\right), l}^{\bar{h} h}=\sum_{q, r=1}^{s_{h}} \kappa_{q r l}^{\bar{h} h} v_{j_{1} q}^{\downarrow} v_{j_{2} r}^{\uparrow} .
$$

Then (D1) becomes

$$
\left(c_{j_{1} \downarrow j_{2} \uparrow}^{i_{1} \uparrow i_{2} \downarrow}\right)_{p \bar{p} \bar{h} h}=\sum_{k=1}^{s_{p \bar{p}}} \sum_{l=1}^{s_{h \bar{h}}} \lambda_{k l 1}^{p \bar{p} \bar{h} h} w_{\left(i_{1} i_{2}\right), k} w_{\left(j_{1}, j_{2}\right), l}
$$

which can be written in matrix form as

$$
\begin{aligned}
\left(C_{\downarrow \uparrow}^{\uparrow \downarrow}\right)_{p \bar{p} \bar{h} h} & =W_{p \bar{p}} \Lambda^{p \bar{p} \bar{h} h} W_{\bar{h} h}^{T} \\
& =W_{p \bar{p}} X \bar{\Lambda} \bar{\Lambda}^{p \bar{p} \bar{h} h} Y^{T} W_{\bar{h} h}^{T} \\
& =\bar{W}_{p \bar{p}} \bar{\Lambda}^{p \bar{p} \bar{h} h} \bar{W}_{\bar{h} h}^{T}
\end{aligned}
$$

where $\bar{\Lambda}^{p \bar{p} \bar{h} h}$ is diagonal, $X$ and $Y$ are unitary, $\bar{W}_{p \bar{p}}=W_{p \bar{p}} X$, $\bar{W}_{\bar{h} h}=W_{\bar{h} h} Y$ and the row indices of $W_{p \bar{p}}$ and $W_{\bar{h} h}$ are $\left(i_{1} i_{2}\right)$ and $\left(j_{1} j_{2}\right)$ pairs, respectively. Now, expression (D5) has SVD form, but it is an actual SVD only if the columns of $\bar{W}_{p \bar{p}}$ and $\bar{W}_{\bar{h} h}$ are orthogonal. Since $X$ and $Y$ are unitary, this is the case if the columns of $W_{p \bar{p}}$ and $W_{\bar{h} h}$, corresponding to matrix slices of tensors $w^{p \bar{p}}$ and $w^{\bar{h} h}$, are orthogonal. Now, using the fact the columns of matrices $u^{\sigma}$ are orthogonal, the matrix slices of $w^{p \bar{p}}$ are orthogonal if the matrix slices of $\kappa^{p \bar{p}}$ are, and similarly for $v^{\sigma}, w^{\bar{h} h}$ and $\kappa_{q r l}^{\bar{h} h}$.

Furthermore, the decompositions (D2) and (D3) are also closely related to SVD's. For instance, for a fixed $k$, (D2) has the matrix form

$$
\begin{aligned}
\tilde{W}_{k}^{p \bar{p}} & =U^{\uparrow} K_{k}\left(U^{\downarrow}\right)^{T} \\
& =U^{\uparrow} R_{k} \bar{K}_{k} S_{k}^{T}\left(U^{\downarrow}\right)^{T} \\
& =U_{k}^{\uparrow} \bar{K}_{k}\left(U_{k}^{\downarrow}\right)^{T}
\end{aligned}
$$

where $\bar{K}_{k}$ is diagonal, $U_{k}^{\uparrow}=U^{\uparrow} R_{k}, U_{k}^{\downarrow}=U^{\downarrow} S_{k}$ and $R_{k}$ and $S_{k}$ are unitary.

Therefore, if the matrix slices of the $\kappa$ tensors are orthogonal, (D1) is related to a combination of SVD's only by a few rotations and thus, for that partition of the indices, that combination of SVD is the optimal decomposition. However, since $s_{p \bar{p}}$ and $s_{h \bar{h}}$ are not equal in general, $\lambda$ is not diagonal, and the orthogonality condition for the $\kappa$ tensors is not necessarily satisfied, the decomposition (D1) is less compact than its corresponding SVD form. The other decompositions of sections VII all have the same structure and can be written similarly as combinations of SVD's using internal rotations. Although, because of those rotations, they are not individually as compact as possible, their more general form allows tensors to be shared between decompositions, and therefore the STTN structure to be globally compact.

\section{E. Computational complexity}

The computational complexity in evaluating the generalized $\mathrm{CC}$ equations of section $\mathrm{VI}$ depends on the term

$$
\sum_{k l m n} V_{k l m n} c_{m n j_{1} j_{2}}^{k l i_{1} i_{2}}
$$

in Eq. (40). More specifically, assuming the decompositions provided in Appendix $\mathrm{C}$ are used, the complexity depends on the contributions in which the summed indices have the largest entanglement with the external ones, namely, when each index in $\{k, l, m, n\}$ is connected by a $\kappa$ tensor to an index in $\left\{i_{1}, i_{2}, j_{1}, j_{2}\right\}$. Those contributions have the form

$$
\begin{aligned}
R_{j_{1} j_{2}}^{i_{1} i_{2}}= & V_{n_{1} n_{2} n_{3} n_{4}} \mu_{m_{1} m_{2}} \lambda_{l_{1} l_{2} m_{1}} \lambda_{l_{3} l_{4} m_{2}} \kappa_{k_{1} k_{2} l_{1}} \kappa_{k_{3} k_{4} l_{2}} \\
& \times \kappa_{k_{5} k_{6} l_{3}} \kappa_{k_{7} k_{8} l_{4}} u_{n_{1} k_{1}} u_{n_{2} k_{3}} u_{i_{1} k_{5}} u_{i_{2} k_{7}} \\
& \times v_{n_{3} k_{6}} v_{n_{4} k_{8}} v_{j_{1} k_{2}} v_{j_{2} k_{4}},
\end{aligned}
$$

where we have used Einstein's notation for the sums. To simplify the scaling analysis, we will assume that each $n_{i}$ index takes $N$ values, the $k_{i}$ indices take $s_{1}$ values, the $l_{i}$ indices, $s_{2}$ values and the $m_{i}$ indices, $s_{4}$ values. We also assume no memory limitation. Now, after summing over $n_{1}$, we obtain

$$
\begin{aligned}
R_{j_{1} j_{2}}^{i_{1} i_{2}}= & (V u)_{n_{2} n_{3} n_{4}}^{k_{1}} \mu_{m_{1} m_{2}} \lambda_{l_{1} l_{2} m_{1}} \lambda_{l_{3} l_{4} m_{2}} \kappa_{k_{1} k_{2} l_{1}} \kappa_{k_{3} k_{4} l_{2}} \\
& \times \kappa_{k_{5} k_{6} l_{3}} \kappa_{k_{7} k_{8} l_{4}} u_{n_{2} k_{3}} u_{i_{1} k_{5}} u_{i_{2} k_{7}} \\
& \times v_{n_{3} k_{6}} v_{n_{4} k_{8}} v_{j_{1} k_{2}} v_{j_{2} k_{4}}
\end{aligned}
$$

where each of the $N^{3} s_{1}$ elements of $(V u)$ takes $O(N)$ operations to compute, and thus $(V u)$ takes $O\left(N^{4} s_{1}\right)$ operations to compute. Then, after summing over $n_{2}$, we obtain

$$
\begin{aligned}
R_{j_{1} j_{2}}^{i_{1} i_{2}}= & \left(V u^{2}\right)_{n_{3} n_{4}}^{k_{1} k_{3}} \mu_{m_{1} m_{2}} \lambda_{l_{1} l_{2} m_{1}} \lambda_{l_{3} l_{4} m_{2}} \kappa_{k_{1} k_{2} l_{1}} \kappa_{k_{3} k_{4} l_{2}} \\
& \times \kappa_{k_{5} k_{6} l_{3}} \kappa_{k_{7} k_{8} l_{4}} u_{i_{1} k_{5}} u_{i_{2} k_{7}} \\
& \times v_{n_{3} k_{6}} v_{n_{4} k_{8}} v_{j_{1} k_{2}} v_{j_{2} k_{4}}
\end{aligned}
$$

where $\left(V u^{2}\right)$ takes $O\left(N^{3} s_{1}^{2}\right)$ additional operations to compute. Similarly, the summation over $n_{3}$ and $n_{4}$ take respec- 
tively $O\left(N^{2} s_{1}^{3}\right)$ and $O\left(N s_{1}^{4}\right)$ additional operations. We then obtain

$$
\begin{aligned}
R_{j_{1} j_{2}}^{i_{1} i_{2}}= & F_{k_{6} k_{8}}^{k_{1} k_{3}} \mu_{m_{1} m_{2}} \lambda_{l_{1} l_{2} m_{1}} \lambda_{l_{3} l_{4} m_{2}} \kappa_{k_{1} k_{2} l_{1}} \kappa_{k_{3} k_{4} l_{2}} \\
& \times \kappa_{k_{5} k_{6} l_{3}} \kappa_{k_{7} k_{8} l_{4}} u_{i_{1} k_{5}} u_{i_{2} k_{7}} v_{j_{1} k_{2}} v_{j_{2} k_{4}}
\end{aligned}
$$

where $F$ thus takes $O\left(N^{4} s_{1}\right)$ operations to compute, since $N \geq s_{1}$. Then, summing over $k_{1}$, we obtain

$$
\begin{aligned}
R_{j_{1} j_{2}}^{i_{1} i_{2}}= & (F \kappa)_{k_{6} k_{8}}^{k_{2} l_{1}, k_{3}} \mu_{m_{1} m_{2}} \lambda_{l_{1} l_{2} m_{1}} \lambda_{l_{3} l_{4} m_{2}} \kappa_{k_{3} k_{4} l_{2}} \\
& \times \kappa_{k_{5} k_{6} l_{3}} \kappa_{k_{7} k_{8} l_{4}} u_{i_{1} k_{5}} u_{i_{2} k_{7}} v_{j_{1} k_{2}} v_{j_{2} k_{4}}
\end{aligned}
$$

where $(F \kappa)$ takes $O\left(s_{1}^{5} s_{2}\right)$ additional operations to compute. After the summation over $k_{3}$, we obtain

$$
\begin{aligned}
R_{j_{1} j_{2}}^{i_{1} i_{2}}= & \left(F \kappa^{2}\right)_{k_{6} k_{8}}^{k_{2} l_{1}, k_{4} l_{2}} \mu_{m_{1} m_{2}} \lambda_{l_{1} l_{2} m_{1}} \lambda_{l_{3} l_{4} m_{2}} \\
& \times \kappa_{k_{5} k_{6} l_{3}} \kappa_{k_{7} k_{8} l_{4}} u_{i_{1} k_{5}} u_{i_{2} k_{7}} v_{j_{1} k_{2}} v_{j_{2} k_{4}},
\end{aligned}
$$

where $\left(F \kappa^{2}\right)$ takes $O\left(s_{1}^{5} s_{2}^{2}\right)$ additional operations to compute. Similarly, the sums over $k_{6}$ and $k_{8}$ take respectively $O\left(s_{1}^{5} s_{2}^{3}\right)$ and $O\left(s_{1}^{5} s_{2}^{4}\right)$ additional operations to compute. We then obtain

$$
\begin{aligned}
R_{j_{1} j_{2}}^{i_{1} i_{2}}= & G_{k_{5} l_{3}, k_{7} l_{4}}^{k_{2} l_{1}, k_{4} l_{2}} \mu_{m_{1} m_{2}} \lambda_{l_{1} l_{2} m_{1}} \lambda_{l_{3} l_{4} m_{2}} u_{i_{1} k_{5}} u_{i_{2} k_{7}} \\
& \times v_{j_{1} k_{2}} v_{j_{2} k_{4}}
\end{aligned}
$$

* dominic.bergeron@usherbrooke.ca

1 M. Troyer and U.-J. Wiese, Phys. Rev. Lett. 94, 170201 (2005)

${ }^{2}$ F. Coester and H. Kümmel, Nuclear Physics 17, 477 (1960)

3 J. Č́ízek, The Journal of Chemical Physics 45, 4256 (1966) https://doi.org/10.1063/1.1727484

4 R. J. Bartlett and M. Musiał, Rev. Mod. Phys. 79, 291 (2007)

5 R. J. Bartlett, Annual Review of Physical Chemistry 32, 359 (1981) https://doi.org/10.1146/annurev.pc.32.100181.002043

6 I. W. Bulik, T. M. Henderson, and G. E. Scuseria, Journal of Chemical Theory and Computation 11, 3171 (2015). pMID: 26575754, https://doi.org/10.1021/acs.jctc.5b00422

7 D. I. Lyakh, M. Musiał, V. F. Lotrich, and R. J. Bartlett, Chemical Reviews 112, 182 (2012), pMID: 22220988, https://doi.org/10.1021/cr2001417

8 E. Krotscheck, H. Kümmel, and J. G. Zabolitzky, Phys. Rev. A 22, 1243 (1980)

9 Y. Xian, Phys. Rev. A 77, 042103 (2008)

${ }^{10}$ E. Neuscamman, The Journal of Chemical Physics 139, 181101 (2013) https://doi.org/10.1063/1.4829536 where $G$ takes $O\left(s_{1}^{5} s_{2}^{4}\right)$ additional operations to compute. Now, if we the sum over $k_{2}$, we obtain

$$
R_{j_{1} j_{2}}^{i_{1} i_{2}}=(G v)_{k_{5} l_{3}, k_{7} l_{4}}^{j_{1} l_{1}, k_{4} l_{2}} \mu_{m_{1} m_{2}} \lambda_{l_{1} l_{2} m_{1}} \lambda_{l_{3} l_{4} m_{2}} u_{i_{1} k_{5}} u_{i_{2} k_{7}} v_{j_{2} k_{4}},
$$

where $(G v)$ takes $O\left(N s_{1}^{4} s_{2}^{4}\right)$ additional operations to compute. Then, after summing over $k_{4}$, we obtain

$$
R_{j_{1} j_{2}}^{i_{1} i_{2}}=\left(G v^{2}\right)_{k_{5} l_{3}, k_{7} l_{4}}^{j_{1} l_{1}, j_{2} l_{2}} \mu_{m_{1} m_{2}} \lambda_{l_{1} l_{2} m_{1}} \lambda_{l_{3} l_{4} m_{2}} u_{i_{1} k_{5}} u_{i_{2} k_{7}},
$$

where $\left(G v^{2}\right)$ takes $O\left(N^{2} s_{1}^{3} s_{2}^{4}\right)$ additional operations to compute. Similarly, the sums over $k_{5}$ and $k_{7}$ take respectively $O\left(N^{3} s_{1}^{2} s_{2}^{4}\right)$ and $O\left(N^{4} s_{1} s_{2}^{4}\right)$ additional operations. We thus obtain

$$
R_{j_{1} j_{2}}^{i_{1} i_{2}}=J_{i_{1} l_{3}, i_{2} l_{4}}^{j_{1} l_{1}, j_{2} l_{2}} \mu_{m_{1} m_{2}} \lambda_{l_{1} l_{2} m_{1}} \lambda_{l_{3} l_{4} m_{2}}
$$

where $J$ takes $O\left(N^{4} s_{1} s_{2}^{4}\right)$ additional operations to compute. Now, after summing over $l_{1}$ and $l_{2}$, we obtain

$$
R_{j_{1} j_{2}}^{i_{1} i_{2}}=(J \lambda)_{i_{1} l_{3}, i_{2} l_{4}}^{j_{1} j_{2} m_{1}} \mu_{m_{1} m_{2}} \lambda_{l_{3} l_{4} m_{2}},
$$

where $(J \lambda)$ takes $O\left(N^{4} s_{2}^{4} s_{4}\right)$ additional operations to compute, and after summing over $l_{3}$ and $l_{4}$, we obtain

$$
R_{j_{1} j_{2}}^{i_{1} i_{2}}=\left(J \lambda^{2}\right)_{i_{1} i_{2} m_{2}}^{j_{1} j_{2} m_{1}} \mu_{m_{1} m_{2}}
$$

where $\left(J \lambda^{2}\right)$ takes $O\left(N^{4} s_{2}^{2} s_{4}^{2}\right)$ additional operations to compute. Finally, the sums over $m_{1}$ and $m_{2}$ to obtain $R$ take $O\left(N^{4} s_{4}^{2}\right)$ additional operations.

We therefore obtain $O\left(s_{1}^{5} s_{2}^{4}\right)+O\left(N^{4} s_{1} s_{2}^{4}\right)+O\left(N^{4} s_{2}^{4} s_{4}\right)+$ $O\left(N^{4} s_{2}^{2} s_{4}^{2}\right)=O\left(N^{4} s_{1} s_{2}^{4}\right)+O\left(N^{4} s_{2}^{4} s_{4}\right)+O\left(N^{4} s_{2}^{2} s_{4}^{2}\right)$ operations in total, where the simplification comes from using $N \geq s_{1}$, and we cannot simplify further without knowing the complexity of $s_{1}, s_{2}$ and $s_{4}$.
11 Y. Qiu, T. M. Henderson, J. Zhao, and G. E. Scuseria, The Journal of Chemical Physics 147, 064111 (2017) https://doi.org/10.1063/1.4991020

12 J. A. Gomez, T. M. Henderson, and G. E. Scuseria, The Journal of Chemical Physics 150, 144108 (2019) https://doi.org/10.1063/1.5085314

13 R. Orùs, Annals of Physics 349, 117 (2014)

14 S.-J. Ran, E. Tirrito, C. Peng, X. Chen, L. Tagliacozzo, G. Su, and M. Lewenstein, "Lecture Notes of Tensor Network Contractions," (2019), arXiv:1708.09213.

15 P. Corboz and G. Vidal, Phys. Rev. B 80, 165129 (2009)

${ }^{16}$ H. J. Changlani, J. M. Kinder, C. J. Umrigar, and G. K.-L. Chan, Phys. Rev. B 80, 245116 (2009)

17 K. H. Marti, B. Bauer, M. Reiher, M. Troyer, and F. Verstraete, New Journal of Physics 12, 103008 (2010)

18 G. K.-L. Chan and S. Sharma, Annual Review of Physical Chemistry 62, 465 (2011),

https://doi.org/10.1146/annurev-physchem-032210-103338

19 D. Poilblanc, P. Corboz, N. Schuch, and J. I. Cirac, 
Phys. Rev. B 89, 241106 (2014).

20 P. Czarnik and J. Dziarmaga, Phys. Rev. B 90, 035144 (2014)

${ }^{21}$ E. Zohar, M. Burrello, T. B. Wahl, and J. I. Cirac, Annals of Physics 363, 385 (2015)

22 S. Szalay, M. Pfeffer, V. Murg, G. Barcza, F. Verstraete, R. Schneider, and O. Legeza, International Journal of Quantum Chemistry 115, 1342 (2015) https://onlinelibrary.wiley.com/doi/pdf/10.1002/qua.24898

23 H.-H. Zhao, K. Ido, S. Morita, and M. Imada, Phys. Rev. B 96, 085103 (2017).

24 U. Benedikt, K.-H. Böhm, and A. A. Auer, The Journal of Chemical Physics 139, 224101 (2013) https://doi.org/10.1063/1.4833565

25 E. G. Hohenstein, R. M. Parrish, C. D. Sherrill, and T. J. Martínez, The Journal of Chemical Physics 137, 221101 (2012). https://doi.org/10.1063/1.4768241

26 E. G. Hohenstein, S. I. L. Kokkila, R. M. Parrish, and T. J. Martínez, The Journal of Physical Chemistry B 117, 12972 (2013) pMID: 23964979, https://doi.org/10.1021/jp4021905

${ }^{27}$ E. G. Hohenstein, S. I. L. Kokkila, R. M. Parrish, and T. J. Martínez, The Journal of Chemical Physics 138, 124111 (2013). https://doi.org/10.1063/1.4795514

28 R. M. Parrish, C. D. Sherrill, E. G. Hohenstein, S. I. L. Kokkila, and T. J. Martínez, The Journal of Chemical Physics 140, 181102 (2014) https://doi.org/10.1063/1.4876016

29 R. Schutski, J. Zhao, T. M. Henderson, and G. E. Scuseria, The Journal of Chemical Physics 147, 184113 (2017), https://doi.org/10.1063/1.4996988

30 A. Tichai, R. Schutski, G. E. Scuseria, and T. Duguet, Phys. Rev. C 99, 034320 (2019).

31 R. M. Parrish, Y. Zhao, E. G. Hohenstein, and T. J. Martínez, The Journal of Chemical Physics 150, 164118 (2019). https://doi.org/10.1063/1.5092505

32 M. Lesiuk, Journal of Computational Chemistry 40, 1319 (2019). https://onlinelibrary.wiley.com/doi/pdf/10.1002/jcc.25788

33 P.-O. Löwdin, Phys. Rev. 97, 1474 (1955)

34 A. Szabo and N. S. Ostlund, Modern Quantum Chemistry: Introduction to Advanced Electronic Structure Theory (Dover Publications, Mineola, New York, 1996).

35 K. A. Brueckner, Phys. Rev. 97, 1353 (1955)

36 J. Goldstone, Proceedings of the Royal Society of London. Series A 239, 267 (1957) https://royalsocietypublishing.org/doi/pdf/10.1098/rspa.1957.0037

37 D. Zgid, E. Gull, and G. K.-L. Chan, Phys. Rev. B 86, 165128 (2012).

38 A. Go and A. J. Millis, Phys. Rev. B 96, 085139 (2017)

$39 \mathrm{G}$ Bergqvist and E. G. Larsson, IEEE Signal Processing Magazine 27, 151 (2010)

40 M. C. Gutzwiller, Phys. Rev. Lett. 10, 159 (1963)

${ }^{41}$ S. A. Kucharski and R. J. Bartlett, Advances in Quantum Chemistry 18, 281 (1986)

42 See supplemental material for the derivation of the generalized CC equations with projection on double-excitation SD's.

43 D. Poulin, A. Qarry, R. Somma, and F. Verstraete, Phys. Rev. Lett. 106, 170501 (2011)

44 M. B. Hastings, Phys. Rev. B 69, 104431 (2004).

45 U. Benedikt, A. A. Auer, M. Espig, and W. Hackbusch, The Journal of Chemical Physics 134, 054118 (2011), https://doi.org/10.1063/1.3514201 


\section{Supplemental material}

\section{Derivation of the generalized CC equations for the double-excitation projection SD}

Here we derive the generalized CC equations for the projection SD's $\left\langle\phi_{j_{1} j_{2}}^{i_{1} i_{2}}\right|$ using the operator

$$
T=\sum_{i, j} a_{i}^{\dagger} a_{j} \hat{D}_{j}^{i}
$$

where $\hat{D}_{j}^{i}$ is defined as

$$
\hat{D}_{j_{k}}^{i_{k}}\left|\phi_{j_{1} j_{2} \ldots j_{k-1}}^{i_{1} i_{2} \ldots i_{k-1}}\right\rangle=\frac{1}{k} \frac{c_{j_{1} j_{2} \ldots j_{k}}^{i_{1} i_{2} \ldots i_{k}}}{c_{j_{1} j_{2} \ldots j_{k-1}}^{i_{1} \ldots i_{k-1}}}\left|\phi_{j_{1} j_{2} \ldots j_{k-1}}^{i_{1} i_{2} \ldots i_{k-1}}\right\rangle,
$$

the excited particle and hole operators

$$
\begin{gathered}
p_{i}^{\dagger}=\left(1-n_{i}^{\phi}\right) a_{i}^{\dagger}, \\
h_{i}^{\dagger}=n_{i}^{\phi} a_{i},
\end{gathered}
$$

respectively, and the hamiltonian

$$
\begin{aligned}
\hat{H}^{\phi} & =\hat{H}-\langle\phi|\hat{H}| \phi\rangle \\
& =\sum_{i j} t_{i j}^{\phi} p_{i}^{\dagger} p_{j}-\sum_{i j} t_{i j}^{\phi} h_{i}^{\dagger} h_{j}+\sum_{i j} t_{i j}^{\phi}\left(p_{i}^{\dagger} h_{j}^{\dagger}+h . c .\right) \\
& +\frac{1}{4} \sum_{i j k l} V_{i j k l} p_{i}^{\dagger} p_{j}^{\dagger} p_{k} p_{l}+\frac{1}{4} \sum_{i j k l} V_{i j k l} h_{i}^{\dagger} h_{j}^{\dagger} h_{k} h_{l}+\frac{1}{4} \sum_{i j k l} V_{i j k l}\left(p_{i}^{\dagger} p_{j}^{\dagger} h_{k}^{\dagger} h_{l}^{\dagger}+h . c .\right) \\
& +\frac{1}{2} \sum_{i j k l} V_{i j k l}\left(p_{i}^{\dagger} p_{j}^{\dagger} h_{k}^{\dagger} p_{l}+h . c .\right)+\frac{1}{2} \sum_{i j k l} V_{i j k l}\left(h_{i}^{\dagger} h_{j}^{\dagger} p_{k}^{\dagger} h_{l}+\text { h.c. }\right)-\sum_{i j k l} V_{i k j l} p_{i}^{\dagger} h_{j}^{\dagger} h_{k} p_{l},
\end{aligned}
$$

where $V_{i j k l}$ is the antisymmetrized Coulomb interaction $V_{i j k l}=V_{i j k l}^{c}-V_{i j l k}^{c}$, where $V_{i j k l}^{c}$ are Coulomb integrals.

Taking into account the facts that $T$ creates a single particle-hole pair, $H$ can create or destroy at most two pairs, and $\left\langle\phi\left|\hat{H}^{\phi}\right| \phi\right\rangle=0$, the equations written in terms of $H$ and $T$ are

$$
\begin{aligned}
0 & =\left\langle\phi_{j_{1} j_{2}}^{i_{1} i_{2}}\left|e^{-T} \hat{H}^{\phi} e^{T}\right| \phi\right\rangle \\
& =\left\langle\phi_{j_{1} j_{2}}^{i_{1} i_{2}}\left|\left(1-T+\frac{1}{2} T^{2}\right) \hat{H}^{\phi}\left(1+T+\frac{1}{2} T^{2}+\frac{1}{3 !} T^{3}+\frac{1}{4 !} T^{4}\right)\right| \phi\right\rangle \\
0 & =\left\langle\phi_{j_{1} j_{2}}^{i_{1} i_{2}}\left|\hat{H}^{\phi}\right| \phi\right\rangle+\left\langle\phi_{j_{1} j_{2}}^{i_{1} i_{2}}\left|\hat{H}^{\phi} T\right| \phi\right\rangle-\left\langle\phi_{j_{1} j_{2}}^{i_{1} i_{2}}\left|T \hat{H}^{\phi}\right| \phi\right\rangle \\
& +\frac{1}{2}\left\langle\phi_{j_{1} j_{2}}^{i_{1} i_{2}}\left|\hat{H}^{\phi} T^{2}\right| \phi\right\rangle-\left\langle\phi_{j_{1} j_{2}}^{i_{1} i_{2}}\left|T \hat{H}^{\phi} T\right| \phi\right\rangle+\frac{1}{3 !}\left\langle\phi_{j_{1} j_{2}}^{i_{1} i_{2}}\left|\hat{H}^{\phi} T^{3}\right| \phi\right\rangle \\
& -\frac{1}{2}\left\langle\phi_{j_{1} j_{2}}^{i_{1} i_{2}}\left|T \hat{H}^{\phi} T^{2}\right| \phi\right\rangle+\frac{1}{2}\left\langle\phi_{j_{1} j_{2}}^{i_{1} i_{2}}\left|T^{2} \hat{H}^{\phi} T\right| \phi\right\rangle+\frac{1}{4 !}\left\langle\phi_{j_{1} j_{2}}^{i_{1} i_{2}}\left|\hat{H}^{\phi} T^{4}\right| \phi\right\rangle \\
& -\frac{1}{3 !}\left\langle\phi_{j_{1} j_{2}}^{i_{1} i_{2}}\left|T \hat{H}^{\phi} T^{3}\right| \phi\right\rangle+\frac{1}{4}\left\langle\phi_{j_{1} j_{2}}^{i_{1} i_{2}}\left|T^{2} \hat{H}^{\phi} T^{2}\right| \phi\right\rangle .
\end{aligned}
$$


The term $\left\langle\phi_{j_{1} j_{2}}^{i_{1} i_{2}}\left|\hat{H}^{\phi}\right| \phi\right\rangle$ involves the part of $H$ that creates two pairs:

$$
\begin{aligned}
\left\langle\phi_{j_{1} j_{2}}^{i_{1} i_{2}}\left|\hat{H}^{\phi}\right| \phi\right\rangle & =\frac{1}{4} \sum_{k l m n} V_{k l m n}\left\langle\phi\left|h_{j_{2}} p_{i_{2}} h_{j_{1}} p_{i_{1}} p_{k}^{\dagger} p_{l}^{\dagger} h_{m}^{\dagger} h_{n}^{\dagger}\right| \phi\right\rangle \\
& =-\frac{1}{4} \sum_{k l m n} V_{k l m n}\left\langle\phi\left|h_{j_{2}} h_{j_{1}} h_{m}^{\dagger} h_{n}^{\dagger} p_{i_{2}} p_{i_{1}} p_{k}^{\dagger} p_{l}^{\dagger}\right| \phi\right\rangle \\
& =-\frac{1}{4} \sum_{k l m n} V_{k l m n}\left(\delta_{j_{1} m} \delta_{j_{2} n}-\delta_{j_{1} n} \delta_{j_{2} m}\right)\left(\delta_{i_{1} k} \delta_{i_{2} l}-\delta_{i_{1} l} \delta_{i_{2} k}\right)
\end{aligned}
$$

which yields, using the antisymmetry of $V_{k l m n}$,

$$
\left\langle\phi_{j_{1} j_{2}}^{i_{1} i_{2}}\left|\hat{H}^{\phi}\right| \phi\right\rangle=-V_{i_{1} i_{2} j_{1} j_{2}} \cdot
$$

The term $\left\langle\phi_{j_{1} j_{2}}^{i_{1} i_{2}}\left|\hat{H}^{\phi} T\right| \phi\right\rangle$ involves all the terms of $H$ creating one pair:

$$
\begin{aligned}
& \left\langle\phi_{j_{1} j_{2}}^{i_{1} i_{2}}\left|\hat{H}^{\phi} T\right| \phi\right\rangle \\
& =\left\langle\phi\left|h_{j_{2}} p_{i_{2}} h_{j_{1}} p_{i_{1}}\left(\sum_{k l} t_{k l}^{\phi} p_{k}^{\dagger} h_{l}^{\dagger}+\frac{1}{2} \sum_{k l m n} V_{k l m n} p_{k}^{\dagger} p_{l}^{\dagger} h_{m}^{\dagger} p_{n}+\frac{1}{2} \sum_{k l m n} V_{k l m n} h_{k}^{\dagger} h_{l}^{\dagger} p_{m}^{\dagger} h_{n}\right) \sum_{q r} c_{r}^{q} p_{q}^{\dagger} h_{r}^{\dagger}\right| \phi\right\rangle \\
& =\sum_{k l q r} t_{k l}^{\phi} c_{r}^{q}\left\langle\phi\left|h_{j_{2}} p_{i_{2}} h_{j_{1}} p_{i_{1}} p_{k}^{\dagger} h_{l}^{\dagger} p_{q}^{\dagger} h_{r}^{\dagger}\right| \phi\right\rangle+\frac{1}{2} \sum_{k l m n q r} V_{k l m n} c_{r}^{q}\left\langle\phi\left|h_{j_{2}} p_{i_{2}} h_{j_{1}} p_{i_{1}} p_{k}^{\dagger} p_{l}^{\dagger} h_{m}^{\dagger} p_{n} p_{q}^{\dagger} h_{r}^{\dagger}\right| \phi\right\rangle \\
& +\frac{1}{2} \sum_{k l m n q r} V_{k l m n} c_{r}^{q}\left\langle\phi\left|h_{j_{2}} p_{i_{2}} h_{j_{1}} p_{i_{1}} h_{k}^{\dagger} h_{l}^{\dagger} p_{m}^{\dagger} h_{n} p_{q}^{\dagger} h_{r}^{\dagger}\right| \phi\right\rangle \\
& =\sum_{k l q r} t_{k l}^{\phi} c_{r}^{q}\left\langle\phi\left|h_{j_{2}} h_{j_{1}} h_{l}^{\dagger} h_{r}^{\dagger} p_{i_{2}} p_{i_{1}} p_{k}^{\dagger} p_{q}^{\dagger}\right| \phi\right\rangle-\frac{1}{2} \sum_{k l m n q r} V_{k l m n} c_{r}^{q}\left\langle\phi\left|h_{j_{2}} h_{j_{1}} h_{m}^{\dagger} h_{r}^{\dagger} p_{i_{2}} p_{i_{1}} p_{k}^{\dagger} p_{l}^{\dagger} p_{n} p_{q}^{\dagger}\right| \phi\right\rangle \\
& +\frac{1}{2} \sum_{k l m n q r} V_{k l m n} c_{r}^{q}\left\langle\phi\left|h_{j_{2}} h_{j_{1}} h_{k}^{\dagger} h_{l}^{\dagger} h_{n} h_{r}^{\dagger} p_{i_{2}} p_{i_{1}} p_{m}^{\dagger} p_{q}^{\dagger}\right| \phi\right\rangle \\
& =\sum_{k l q r} t_{k l}^{\phi} c_{r}^{q}\left(\delta_{j_{1} l} \delta_{j_{2} r}-\delta_{j_{1} r} \delta_{j_{2} l}\right)\left(\delta_{i_{1} k} \delta_{i_{2} q}-\delta_{i_{1} q} \delta_{i_{2} k}\right)-\frac{1}{2} \sum_{k l m q r} V_{k l m q} c_{r}^{q}\left\langle\phi\left|h_{j_{2}} h_{j_{1}} h_{m}^{\dagger} h_{r}^{\dagger} p_{i_{2}} p_{i_{1}} p_{k}^{\dagger} p_{l}^{\dagger}\right| \phi\right\rangle \\
& +\frac{1}{2} \sum_{k l m q r} V_{k l m r} c_{r}^{q}\left\langle\phi\left|h_{j_{2}} h_{j_{1}} h_{k}^{\dagger} h_{l}^{\dagger} p_{i_{2}} p_{i_{1}} p_{m}^{\dagger} p_{q}^{\dagger}\right| \phi\right\rangle \\
& =\left(t_{i_{1} j_{1}}^{\phi} c_{j_{2}}^{i_{2}}-t_{i_{1} j_{2}}^{\phi} c_{j_{1}}^{i_{2}}-t_{i_{2} j_{1}}^{\phi} c_{j_{2}}^{i_{1}}+t_{i_{2} j_{2}}^{\phi} c_{j_{1}}^{i_{1}}\right) \\
& -\frac{1}{2} \sum_{k l m q r} V_{k l m q} c_{r}^{q}\left(\delta_{j_{1} m} \delta_{j_{2} r}-\delta_{j_{1} r} \delta_{j_{2} m}\right)\left(\delta_{i_{1} k} \delta_{i_{2} l}-\delta_{i_{1} l} \delta_{i_{2} k}\right) \\
& +\frac{1}{2} \sum_{k l m q r} V_{k l m r} c_{r}^{q}\left(\delta_{j_{1} k} \delta_{j_{2} l}-\delta_{j_{1} l} \delta_{j_{2} k}\right)\left(\delta_{i_{1} m} \delta_{i_{2} q}-\delta_{i_{1} q} \delta_{i_{2} m}\right) \\
& =\left(t_{i_{1} j_{1}}^{\phi} c_{j_{2}}^{i_{2}}-t_{i_{1} j_{2}}^{\phi} c_{j_{1}}^{i_{2}}-t_{i_{2} j_{1}}^{\phi} c_{j_{2}}^{i_{1}}+t_{i_{2} j_{2}}^{\phi} c_{j_{1}}^{i_{1}}\right)-\sum_{m q r} V_{i_{1} i_{2} m q} c_{r}^{q}\left(\delta_{j_{1} m} \delta_{j_{2} r}-\delta_{j_{1} r} \delta_{j_{2} m}\right) \\
& +\sum_{m q r} V_{j_{1} j_{2} m r} c_{r}^{q}\left(\delta_{i_{1} m} \delta_{i_{2} q}-\delta_{i_{1} q} \delta_{i_{2} m}\right)
\end{aligned}
$$

$$
\begin{aligned}
\left\langle\phi_{j_{1} j_{2}}^{i_{1} i_{2}}\left|\hat{H}^{\phi} T\right| \phi\right\rangle= & t_{i_{1} j_{1}}^{\phi} c_{j_{2}}^{i_{2}}-t_{i_{1} j_{2}}^{\phi} c_{j_{1}}^{i_{2}}-t_{i_{2} j_{1}}^{\phi} c_{j_{2}}^{i_{1}}+t_{i_{2} j_{2}}^{\phi} c_{j_{1}}^{i_{1}}-\sum_{q}\left(V_{i_{1} i_{2} j_{1} q} c_{j_{2}}^{q}-V_{i_{1} i_{2} j_{2} q} c_{j_{1}}^{q}\right) \\
& +\sum_{r}\left(V_{j_{1} j_{2} i_{1} r} c_{r}^{i_{2}}-V_{j_{1} j_{2} i_{2} r} c_{r}^{i_{1}}\right)
\end{aligned}
$$


The term $\left\langle\phi_{j_{1} j_{2}}^{i_{1} i_{2}}\left|T \hat{H}^{\phi}\right| \phi\right\rangle$ involves only the one-body part of $H$ creating one pair:

$$
\begin{aligned}
\left\langle\phi_{j_{1} j_{2}}^{i_{1} i_{2}}\left|T \hat{H}^{\phi}\right| \phi\right\rangle & =\left\langle\phi\left|h_{j_{2}} p_{i_{2}} h_{j_{1}} p_{i_{1}} \sum_{k l} p_{k}^{\dagger} h_{l}^{\dagger} \hat{D}_{l}^{k} \sum_{q r} t_{q r}^{\phi} p_{q}^{\dagger} h_{r}^{\dagger}\right| \phi\right\rangle \\
& =\frac{1}{2} \sum_{k l q r} \frac{c_{l r}^{k q}}{c_{r}^{q}} t_{q r}^{\phi}\left\langle\phi\left|h_{j_{2}} p_{i_{2}} h_{j_{1}} p_{i_{1}} p_{k}^{\dagger} h_{l}^{\dagger} p_{q}^{\dagger} h_{r}^{\dagger}\right| \phi\right\rangle \\
& =\frac{1}{2} \sum_{k l q r} \frac{c_{l r}^{k q}}{c_{r}^{q}} t_{q r}^{\phi}\left\langle\phi\left|h_{j_{2}} h_{j_{1}} h_{l}^{\dagger} h_{r}^{\dagger} p_{i_{2}} p_{i_{1}} p_{k}^{\dagger} p_{q}^{\dagger}\right| \phi\right\rangle \\
& =\frac{1}{2} \sum_{k l q r} \frac{c_{l r}^{k q}}{c_{r}^{q}} t_{q r}^{\phi}\left(\delta_{j_{1} l} \delta_{j_{2} r}-\delta_{j_{1} r} \delta_{j_{2} l}\right)\left(\delta_{i_{1} k} \delta_{i_{2} q}-\delta_{i_{1} q} \delta_{i_{2} k}\right) \\
& =\frac{1}{2} \sum_{k l q r} \frac{c_{l r}^{k q}}{c_{r}^{q}} t_{q r}^{\phi}\left(\delta_{j_{1} l} \delta_{j_{2} r} \delta_{i_{1} k} \delta_{i_{2} q}-\delta_{j_{1} l} \delta_{j_{2} r} \delta_{i_{1} q} \delta_{i_{2} k}-\delta_{j_{1} r} \delta_{j_{2} l} \delta_{i_{1} k} \delta_{i_{2} q}+\delta_{j_{1} r} \delta_{j_{2} l} \delta_{i_{1} q} \delta_{i_{2} k}\right) \\
& =\frac{1}{2}\left(\frac{c_{j_{1} j_{2}}^{i_{1} i_{2}}}{c_{j_{2}}^{i_{2}}} t_{i_{2} j_{2}}^{\phi}-\frac{c_{j_{1} j_{2}}^{i_{2} i_{1}}}{c_{j_{2}}^{i_{1}}} t_{i_{1} j_{2}}^{\phi}-\frac{c_{j_{j_{2}} i_{1}}^{i_{1} i_{2}}}{c_{j_{1}}^{i_{2}}} t_{i_{2} j_{1}}^{\phi}+\frac{c_{j_{2} j_{1}}^{i_{2} i_{1}}}{c_{j_{1}}^{i_{1}}} t_{i_{1} j_{1}}^{\phi}\right)
\end{aligned}
$$

and thus, using the antisymmetry of $c_{j_{1} j_{2}}^{i_{1} i_{2}}$,

$$
-\left\langle\phi_{j_{1} j_{2}}^{i_{1} i_{2}}\left|T \hat{H}^{\phi}\right| \phi\right\rangle=-\frac{1}{2} c_{j_{1} j_{2}}^{i_{1} i_{2}}\left(\frac{t_{i_{2} j_{2}}^{\phi}}{c_{j_{2}}^{i_{2}}}+\frac{t_{i_{1} j_{2}}^{\phi}}{c_{j_{2}}^{i_{1}}}+\frac{t_{i_{2} j_{1}}^{\phi}}{c_{j_{1}}^{i_{2}}}+\frac{t_{i_{1} j_{1}}^{\phi}}{c_{j_{1}}^{i_{1}}}\right) .
$$

In $\left\langle\phi_{j_{1} j_{2}}^{i_{1} i_{2}}\left|\hat{H}^{\phi} T^{2}\right| \phi\right\rangle$, all the terms that do not create or destroy any pair contribute:

$$
\begin{aligned}
\left\langle\phi_{j_{1} j_{2}}^{i_{1} i_{2}}\right| & H^{\phi} T^{2}|\phi\rangle \\
& =\langle\phi| h_{j_{2}} p_{i_{2}} h_{j_{1}} p_{i_{1}}\left(\sum_{k l} t_{k l}^{\phi} p_{k}^{\dagger} p_{l}-\sum_{k l} t_{k l}^{\phi} h_{k}^{\dagger} h_{l}+\frac{1}{4} \sum_{k l m n} V_{k l m n} p_{k}^{\dagger} p_{l}^{\dagger} p_{m} p_{n}\right. \\
& \left.+\frac{1}{4} \sum_{k l m n} V_{k l m n} h_{k}^{\dagger} h_{l}^{\dagger} h_{m} h_{n}-\sum_{k l m n} V_{k m l n} p_{k}^{\dagger} h_{l}^{\dagger} h_{m} p_{n}\right) \frac{1}{2} \sum_{q r s t} c_{r t}^{q s} p_{q}^{\dagger} h_{r}^{\dagger} p_{s}^{\dagger} h_{t}^{\dagger}|\phi\rangle \\
& =\frac{1}{2} \sum_{k l q r s t} t_{k l}^{\phi} c_{r t}^{q s}\left\langle\phi\left|h_{j_{2}} p_{i_{2}} h_{j_{1}} p_{i_{1}} p_{k}^{\dagger} p_{l} p_{q}^{\dagger} h_{r}^{\dagger} p_{s}^{\dagger} h_{t}^{\dagger}\right| \phi\right\rangle-\frac{1}{2} \sum_{k l q r s t} t_{k l}^{\phi} c_{r t}^{q s}\left\langle\phi\left|h_{j_{2}} p_{i_{2}} h_{j_{1}} p_{i_{1}} h_{k}^{\dagger} h_{l} p_{q}^{\dagger} h_{r}^{\dagger} p_{s}^{\dagger} h_{t}^{\dagger}\right| \phi\right\rangle \\
& +\frac{1}{8} \sum_{k l m n q r s t} V_{k l m n} c_{r t}^{q s}\left\langle\phi\left|h_{j_{2}} p_{i_{2}} h_{j_{1}} p_{i_{1}} p_{k}^{\dagger} p_{l}^{\dagger} p_{m} p_{n} p_{q}^{\dagger} h_{r}^{\dagger} p_{s}^{\dagger} h_{t}^{\dagger}\right| \phi\right\rangle \\
& +\frac{1}{8} \sum_{k l m n q r s t} V_{k l m n} c_{r t}^{q s}\left\langle\phi\left|h_{j_{2}} p_{i_{2}} h_{j_{1}} p_{i_{1}} h_{k}^{\dagger} h_{l}^{\dagger} h_{m} h_{n} p_{q}^{\dagger} h_{r}^{\dagger} p_{s}^{\dagger} h_{t}^{\dagger}\right| \phi\right\rangle \\
& -\frac{1}{2} \sum_{k l m n q r s t} V_{k m l n} c_{r t}^{q s}\left\langle\phi\left|h_{j_{2}} p_{i_{2}} h_{j_{1}} p_{i_{1}} p_{k}^{\dagger} h_{l}^{\dagger} h_{m} p_{n} p_{q}^{\dagger} h_{r}^{\dagger} p_{s}^{\dagger} h_{t}^{\dagger}\right| \phi\right\rangle
\end{aligned}
$$




$$
\begin{aligned}
& \left\langle\phi_{j_{1} j_{2}}^{i_{1} i_{2}}\left|\hat{H}^{\phi} T^{2}\right| \phi\right\rangle \\
& =\frac{1}{2} \sum_{k l q r s t} t_{k l}^{\phi} c_{r t}^{q s}\left\langle\phi\left|h_{j_{2}} h_{j_{1}} h_{r}^{\dagger} h_{t}^{\dagger} p_{i_{2}} p_{i_{1}} p_{k}^{\dagger} p_{l} p_{q}^{\dagger} p_{s}^{\dagger}\right| \phi\right\rangle-\frac{1}{2} \sum_{k l q r s t} t_{k l}^{\phi} c_{r t}^{q s}\left\langle\phi\left|h_{j_{2}} h_{j_{1}} h_{k}^{\dagger} h_{l} h_{r}^{\dagger} h_{t}^{\dagger} p_{i_{2}} p_{i_{1}} p_{q}^{\dagger} p_{s}^{\dagger}\right| \phi\right\rangle \\
& +\frac{1}{8} \sum_{k l m n q r s t} V_{k l m n} c_{r t}^{q s}\left\langle\phi\left|h_{j_{2}} h_{j_{1}} h_{r}^{\dagger} h_{t}^{\dagger} p_{i_{2}} p_{i_{1}} p_{k}^{\dagger} p_{l}^{\dagger} p_{m} p_{n} p_{q}^{\dagger} p_{s}^{\dagger}\right| \phi\right\rangle \\
& +\frac{1}{8} \sum_{k l m n q r s t} V_{k l m n} c_{r t}^{q s}\left\langle\phi\left|h_{j_{2}} h_{j_{1}} h_{k}^{\dagger} h_{l}^{\dagger} h_{m} h_{n} h_{r}^{\dagger} h_{t}^{\dagger} p_{i_{2}} p_{i_{1}} p_{q}^{\dagger} p_{s}^{\dagger}\right| \phi\right\rangle \\
& -\frac{1}{2} \sum_{k l m n q r s t} V_{k m l n} c_{r t}^{q s}\left\langle\phi\left|h_{j_{2}} h_{j_{1}} h_{l}^{\dagger} h_{m} h_{r}^{\dagger} h_{t}^{\dagger} p_{i_{2}} p_{i_{1}} p_{k}^{\dagger} p_{n} p_{q}^{\dagger} p_{s}^{\dagger}\right| \phi\right\rangle \\
& \left\langle\phi_{j_{1} j_{2}}^{i_{1} i_{2}}\left|\hat{H}^{\phi} T^{2}\right| \phi\right\rangle \\
& =\frac{1}{2} \sum_{k l q r s t} t_{k l}^{\phi} c_{r t}^{q s}\left(\delta_{j_{1} r} \delta_{j_{2} t}-\delta_{j_{1} t} \delta_{j_{2} r}\right)\left[\delta_{i_{1} k}\left(\delta_{l q} \delta_{i_{2} s}-\delta_{l s} \delta_{i_{2} q}\right)-\delta_{i_{2} k}\left(\delta_{l q} \delta_{i_{1} s}-\delta_{l s} \delta_{i_{1} q}\right)\right] \\
& -\frac{1}{2} \sum_{k l q r s t} t_{k l}^{\phi} c_{r t}^{q s}\left[\delta_{j_{1} k}\left(\delta_{l r} \delta_{j_{2} t}-\delta_{l t} \delta_{j_{2} r}\right)-\delta_{j_{2} k}\left(\delta_{l r} \delta_{j_{1} t}-\delta_{l t} \delta_{j_{1} r}\right)\right]\left(\delta_{i_{1} q} \delta_{i_{2} s}-\delta_{i_{1} s} \delta_{i_{2} q}\right) \\
& +\frac{1}{8} \sum_{k l m n q r s t} V_{k l m n} c_{r t}^{q s}\left(\delta_{j_{1} r} \delta_{j_{2} t}-\delta_{j_{1} t} \delta_{j_{2} r}\right)\left(\delta_{i_{1} k} \delta_{i_{2} l}-\delta_{i_{1} l} \delta_{i_{2} k}\right)\left(\delta_{n q} \delta_{m s}-\delta_{n s} \delta_{m q}\right) \\
& +\frac{1}{8} \sum_{k l m n q r s t} V_{k l m n} c_{r t}^{q s}\left(\delta_{j_{1} k} \delta_{j_{2} l}-\delta_{j_{1} l} \delta_{j_{2} k}\right)\left(\delta_{n r} \delta_{m t}-\delta_{n t} \delta_{m r}\right)\left(\delta_{i_{1} q} \delta_{i_{2} s}-\delta_{i_{1} s} \delta_{i_{2} q}\right) \\
& -\frac{1}{2} \sum_{k l m n q r s t} V_{k m l n} c_{r t}^{q s}\left[\delta_{j_{1} l}\left(\delta_{m r} \delta_{j_{2} t}-\delta_{m t} \delta_{j_{2} r}\right)-\delta_{j_{2} l}\left(\delta_{m r} \delta_{j_{1} t}-\delta_{m t} \delta_{j_{1} r}\right)\right] \\
& \times\left[\delta_{i_{1} k}\left(\delta_{n q} \delta_{i_{2} s}-\delta_{n s} \delta_{i_{2} q}\right)-\delta_{i_{2} k}\left(\delta_{n q} \delta_{i_{1} s}-\delta_{n s} \delta_{i_{1} q}\right)\right]
\end{aligned}
$$

which becomes, using the antisymmetry of $c_{r t}^{q s}$,

$$
\begin{aligned}
\left\langle\phi_{j_{1} j_{2}}^{i_{1} i_{2}}\left|\hat{H}^{\phi} T^{2}\right| \phi\right\rangle & =2 \sum_{k l q r s t} t_{k l}^{\phi} c_{r t}^{q s} \delta_{j_{1} r} \delta_{j_{2} t}\left(\delta_{i_{1} k} \delta_{l q} \delta_{i_{2} s}+\delta_{i_{2} k} \delta_{l s} \delta_{i_{1} q}\right) \\
& -2 \sum_{k l q r s t} t_{k l}^{\phi} c_{r t}^{q s} \delta_{i_{1} q} \delta_{i_{2} s}\left(\delta_{j_{1} k} \delta_{l r} \delta_{j_{2} t}+\delta_{j_{2} k} \delta_{l t} \delta_{j_{1} r}\right) \\
& -\sum_{k l m n q r s t} V_{k l m n} c_{r t}^{q s} \delta_{j_{1} r} \delta_{j_{2} t} \delta_{i_{1} k} \delta_{i_{2} l} \delta_{n s} \delta_{m q} \\
& -\sum_{k l m n q r s t} V_{k l m n} c_{r t}^{q s} \delta_{j_{1} k} \delta_{j_{2} l} \delta_{n t} \delta_{m r} \delta_{i_{1} q} \delta_{i_{2} s} \\
& -2 \sum_{k l m n q r s t} V_{k m l n} c_{r t}^{q s}\left(\delta_{j_{1} l} \delta_{m r} \delta_{j_{2} t}+\delta_{j_{2} l} \delta_{m t} \delta_{j_{1} r}\right)\left(\delta_{i_{1} k} \delta_{n q} \delta_{i_{2} s}+\delta_{i_{2} k} \delta_{n s} \delta_{i_{1} q}\right)
\end{aligned}
$$

and thus,

$$
\begin{aligned}
\frac{1}{2}\left\langle\phi_{j_{1} j_{2}}^{i_{1} i_{2}}\left|\hat{H}^{\phi} T^{2}\right| \phi\right\rangle & =\sum_{l}\left(t_{i_{1} l}^{\phi} c_{j_{1} j_{2}}^{l i_{2}}+t_{i_{2} l}^{\phi} c_{j_{1} j_{2}}^{i_{1} l}\right)-\sum_{l}\left(t_{j_{1} l}^{\phi} c_{l j_{2}}^{i_{1} i_{2}}+t_{j_{2}}^{\phi} c_{j_{1} l}^{i_{1} i_{2}}\right) \\
& -\frac{1}{2} \sum_{m n} V_{i_{1} i_{2} m n} c_{j_{1} j_{2}}^{m n}-\frac{1}{2} \sum_{m n} V_{j_{1} j_{2} m n} c_{m n}^{i_{1} i_{2}} \\
& -\sum_{m n}\left(V_{i_{1} m j_{1} n} c_{m j_{2}}^{n i_{2}}+V_{i_{2} m j_{1} n} c_{m j_{2}}^{i_{1} n}+V_{i_{1} m j_{2} n} c_{j_{1} m}^{n i_{2}}+V_{i_{2} m j_{2} n} c_{j_{1} m}^{i_{1} n}\right) .
\end{aligned}
$$


Now, in $\left\langle\phi_{j_{1} j_{2}}^{i_{1} i_{2}}\left|T \hat{H}^{\phi} T\right| \phi\right\rangle$, only the terms acting on a single pair and that do not create or destroy any pair contribute:

$$
\begin{aligned}
& \left\langle\phi_{j_{1} j_{2}}^{i_{1} i_{2}}\left|T \hat{H}^{\phi} T\right| \phi\right\rangle \\
& =\langle\phi| h_{j_{2}} p_{i_{2}} h_{j_{1}} p_{i_{1}} \sum_{k l} p_{k}^{\dagger} h_{l}^{\dagger} \hat{D}_{l}^{k} \\
& \times\left(\sum_{q r} t_{q r}^{\phi} p_{q}^{\dagger} p_{r}-\sum_{q r} t_{q r}^{\phi} h_{q}^{\dagger} h_{r}-\sum_{q q_{1} r r_{1}} V_{q r_{1} r q_{1}} p_{q}^{\dagger} h_{r}^{\dagger} h_{r_{1}} p_{q_{1}}\right) \sum_{s t} c_{t}^{s} p_{s}^{\dagger} h_{t}^{\dagger}|\phi\rangle \\
& =\left\langle\phi\left|h_{j_{2}} p_{i_{2}} h_{j_{1}} p_{i_{1}} \sum_{k l} p_{k}^{\dagger} h_{l}^{\dagger} \hat{D}_{l}^{k} \sum_{q r} t_{q r}^{\phi} p_{q}^{\dagger} p_{r} \sum_{s t} c_{t}^{s} p_{s}^{\dagger} h_{t}^{\dagger}\right| \phi\right\rangle \\
& -\left\langle\phi\left|h_{j_{2}} p_{i_{2}} h_{j_{1}} p_{i_{1}} \sum_{k l} p_{k}^{\dagger} h_{l}^{\dagger} \hat{D}_{l}^{k} \sum_{q r} t_{q r}^{\phi} h_{q}^{\dagger} h_{r} \sum_{s t} c_{t}^{s} p_{s}^{\dagger} h_{t}^{\dagger}\right| \phi\right\rangle \\
& -\left\langle\phi\left|h_{j_{2}} p_{i_{2}} h_{j_{1}} p_{i_{1}} \sum_{k l} p_{k}^{\dagger} h_{l}^{\dagger} \hat{D}_{l}^{k} \sum_{q q_{1} r r_{1}} V_{q r_{1} r q_{1}} p_{q}^{\dagger} h_{r}^{\dagger} h_{r_{1}} p_{q_{1}} \sum_{s t} c_{t}^{s} p_{s}^{\dagger} h_{t}^{\dagger}\right| \phi\right\rangle \\
& =\sum_{k l q r s t} t_{q r}^{\phi} c_{t}^{s}\left\langle\phi\left|h_{j_{2}} p_{i_{2}} h_{j_{1}} p_{i_{1}} p_{k}^{\dagger} h_{l}^{\dagger} \hat{D}_{l}^{k} p_{q}^{\dagger} p_{r} p_{s}^{\dagger} h_{t}^{\dagger}\right| \phi\right\rangle \\
& -\sum_{k l q r s t} t_{q r}^{\phi} c_{t}^{s}\left\langle\phi\left|h_{j_{2}} p_{i_{2}} h_{j_{1}} p_{i_{1}} p_{k}^{\dagger} h_{l}^{\dagger} \hat{D}_{l}^{k} h_{q}^{\dagger} h_{r} p_{s}^{\dagger} h_{t}^{\dagger}\right| \phi\right\rangle \\
& -\sum_{k l q q_{1} r r_{1} s t} V_{q r_{1} r q_{1}} c_{t}^{s}\left\langle\phi\left|h_{j_{2}} p_{i_{2}} h_{j_{1}} p_{i_{1}} p_{k}^{\dagger} h_{l}^{\dagger} \hat{D}_{l}^{k} p_{q}^{\dagger} h_{r}^{\dagger} h_{r_{1}} p_{q_{1}} p_{s}^{\dagger} h_{t}^{\dagger}\right| \phi\right\rangle \\
& =\sum_{k l q r s t} t_{q r}^{\phi} c_{t}^{s} \delta_{r s}\left\langle\phi\left|h_{j_{2}} p_{i_{2}} h_{j_{1}} p_{i_{1}} p_{k}^{\dagger} h_{l}^{\dagger} \hat{D}_{l}^{k} p_{q}^{\dagger} h_{t}^{\dagger}\right| \phi\right\rangle \\
& -\sum_{k l q r s t} t_{q r}^{\phi} c_{t}^{s} \delta_{r t}\left\langle\phi\left|h_{j_{2}} p_{i_{2}} h_{j_{1}} p_{i_{1}} p_{k}^{\dagger} h_{l}^{\dagger} \hat{D}_{l}^{k} p_{s}^{\dagger} h_{q}^{\dagger}\right| \phi\right\rangle \\
& -\sum_{k l q q_{1} r r_{1} s t} V_{q r_{1} r q_{1}} c_{t}^{s} \delta_{q_{1} s} \delta_{r_{1} t}\left\langle\phi\left|h_{j_{2}} p_{i_{2}} h_{j_{1}} p_{i_{1}} p_{k}^{\dagger} h_{l}^{\dagger} \hat{D}_{l}^{k} p_{q}^{\dagger} h_{r}^{\dagger}\right| \phi\right\rangle \\
& \left\langle\phi_{j_{1} j_{2}}^{i_{1} i_{2}}\left|T \hat{H}^{\phi} T\right| \phi\right\rangle=\frac{1}{2} \sum_{k l q r t} \frac{c_{l t}^{k q}}{c_{t}^{q}} t_{q r}^{\phi} c_{t}^{r}\left\langle\phi\left|h_{j_{2}} p_{i_{2}} h_{j_{1}} p_{i_{1}} p_{k}^{\dagger} h_{l}^{\dagger} p_{q}^{\dagger} h_{t}^{\dagger}\right| \phi\right\rangle \\
& -\frac{1}{2} \sum_{k l q r s} \frac{c_{l q}^{k s}}{c_{q}^{s}} t_{q r}^{\phi} c_{r}^{s}\left\langle\phi\left|h_{j_{2}} p_{i_{2}} h_{j_{1}} p_{i_{1}} p_{k}^{\dagger} h_{l}^{\dagger} p_{s}^{\dagger} h_{q}^{\dagger}\right| \phi\right\rangle \\
& -\frac{1}{2} \sum_{k l q q_{1} r r_{1}} \frac{c_{l r}^{k q}}{c_{r}^{q}} V_{q r_{1} r q_{1}} c_{r_{1}}^{q_{1}}\left\langle\phi\left|h_{j_{2}} p_{i_{2}} h_{j_{1}} p_{i_{1}} p_{k}^{\dagger} h_{l}^{\dagger} p_{q}^{\dagger} h_{r}^{\dagger}\right| \phi\right\rangle \\
& =\frac{1}{2} \sum_{k l q r t} \frac{c_{l t}^{k q}}{c_{t}^{q}} t_{q r}^{\phi} c_{t}^{r}\left\langle\phi\left|h_{j_{2}} h_{j_{1}} h_{l}^{\dagger} h_{t}^{\dagger} p_{i_{2}} p_{i_{1}} p_{k}^{\dagger} p_{q}^{\dagger}\right| \phi\right\rangle \\
& -\frac{1}{2} \sum_{k l q r s} \frac{c_{l q}^{k s}}{c_{q}^{s}} t_{q r}^{\phi} c_{r}^{s}\left\langle\phi\left|h_{j_{2}} h_{j_{1}} h_{l}^{\dagger} h_{q}^{\dagger} p_{i_{2}} p_{i_{1}} p_{k}^{\dagger} p_{s}^{\dagger}\right| \phi\right\rangle \\
& -\frac{1}{2} \sum_{k l q q_{1} r r_{1}} \frac{c_{l r}^{k q}}{c_{r}^{q}} V_{q r_{1} r q_{1}} c_{r_{1}}^{q_{1}}\left\langle\phi\left|h_{j_{2}} h_{j_{1}} h_{l}^{\dagger} h_{r}^{\dagger} p_{i_{2}} p_{i_{1}} p_{k}^{\dagger} p_{q}^{\dagger}\right| \phi\right\rangle
\end{aligned}
$$




$$
\begin{aligned}
\left\langle\phi_{j_{1} j_{2}}^{i_{1} i_{2}}\left|T \hat{H}^{\phi} T\right| \phi\right\rangle & =\frac{1}{2} \sum_{k l q r t} \frac{c_{l t}^{k q}}{c_{t}^{q}} t_{q r}^{\phi} c_{t}^{r}\left(\delta_{l j_{1}} \delta_{t j_{2}}-\delta_{l j_{2}} \delta_{t j_{1}}\right)\left(\delta_{k i_{1}} \delta_{q i_{2}}-\delta_{k i_{2}} \delta_{q i_{1}}\right) \\
& -\frac{1}{2} \sum_{k l q r s} \frac{c_{l q}^{k s}}{c_{q}^{s}} t_{q r}^{\phi} c_{r}^{s}\left(\delta_{l j_{1}} \delta_{q j_{2}}-\delta_{l j_{2}} \delta_{q j_{1}}\right)\left(\delta_{k i_{1}} \delta_{s i_{2}}-\delta_{k i_{2}} \delta_{s i_{1}}\right) \\
& -\frac{1}{2} \sum_{k l q q_{1} r r_{1}} \frac{c_{l r}^{k q}}{c_{r}^{q}} V_{q r_{1} r q_{1}} c_{r_{1}}^{q_{1}}\left(\delta_{l j_{1}} \delta_{r j_{2}}-\delta_{l j_{2}} \delta_{r j_{1}}\right)\left(\delta_{k i_{1}} \delta_{q i_{2}}-\delta_{k i_{2}} \delta_{q i_{1}}\right) \\
& =\frac{1}{2} \sum_{r}\left(\frac{c_{j_{1} j_{2}}^{i_{1} i_{2}}}{c_{j_{2}}^{i_{2}}} t_{i_{2} r}^{\phi} c_{j_{2}}^{r}-\frac{c_{j_{1} j_{2}}^{i_{2} i_{1}}}{c_{j_{2}}^{i_{1}}} t_{i_{1} r}^{\phi} c_{j_{2}}^{r}-\frac{c_{j_{2} j_{1}}^{i_{1} i_{2}}}{c_{j_{1}}^{i_{2}}} t_{i_{2} r}^{\phi} c_{j_{1}}^{r}+\frac{c_{j_{2} j_{1}}^{i_{2} i_{1}}}{c_{j_{1}}^{i_{1}}} t_{i_{1} r}^{\phi} c_{j_{1}}^{r}\right) \\
& -\frac{1}{2} \sum_{r}\left(\frac{c_{j_{1} j_{2}}^{i_{1} i_{2}}}{c_{j_{2}}^{i_{2}}} t_{j_{2} r}^{\phi} c_{r}^{i_{2}}-\frac{c_{j_{1} j_{2}}^{i_{2} i_{1}}}{c_{j_{2}}^{i_{1}}} t_{j_{2} r}^{\phi} c_{r}^{i_{1}}-\frac{c_{j_{2} j_{1}}^{i_{1} i_{2}}}{c_{j_{2}}^{i_{2}}} t_{j_{1} r}^{\phi} c_{r}^{i_{2}}+\frac{c_{j_{2} j_{1}}^{i_{2} i_{1}}}{c_{j_{1}}^{i_{1}}} t_{j_{1} r}^{\phi} c_{r}^{i_{1}}\right) \\
& -\frac{1}{2} \sum_{q_{1} r_{1}}\left(\frac{c_{j_{1} j_{2}}^{i_{1} i_{2}}}{c_{j_{2}}^{i_{2}}} V_{i_{2} r_{1} j_{2} q_{1}} c_{r_{1}}^{q_{1}}-\frac{c_{j_{1} j_{2}}^{i_{2} i_{1}}}{c_{j_{2}}^{i_{1}}} V_{i_{1} r_{1} j_{2} q_{1}} c_{r_{1}}^{q_{1}}-\frac{c_{j_{2} j_{1}}^{i_{1} i_{2}}}{c_{j_{1}}^{i_{2}}} V_{i_{2} r_{1} j_{1} q_{1}} c_{r_{1}}^{q_{1}}+\frac{c_{j_{2} j_{1}}^{i_{2} i_{1}}}{c_{j_{1}}^{i_{1}}} V_{i_{1} r_{1} j_{1} q_{1}} c_{r_{1}}^{q_{1}}\right)
\end{aligned}
$$

which becomes, using the antisymmetry of $c_{j_{1} j_{2}}^{i_{1} i_{2}}$,

$$
\begin{aligned}
-\left\langle\phi_{j_{1} j_{2}}^{i_{1} i_{2}}\left|T \hat{H}^{\phi} T\right| \phi\right\rangle & =c_{j_{1} j_{2}}^{i_{1} i_{2}}\left[-\frac{1}{2} \sum_{r}\left(\frac{t_{i_{2} r}^{\phi} c_{j_{2}}^{r}}{c_{j_{2}}^{i_{2}}}+\frac{t_{i_{1} r}^{\phi} c_{j_{2}}^{r}}{c_{j_{2}}^{i_{1}}}+\frac{t_{i_{2} r}^{\phi} c_{j_{1}}^{r}}{c_{j_{1}}^{i_{2}}}+\frac{t_{i_{1} r}^{\phi} c_{j_{1}}^{r}}{c_{j_{1}}^{i_{1}}}\right)\right. \\
& +\frac{1}{2} \sum_{r}\left(\frac{t_{j_{2} r}^{\phi} c_{r}^{i_{2}}}{c_{j_{2}}^{i_{2}}}+\frac{t_{j_{2} r}^{\phi} c_{r}^{i_{1}}}{c_{j_{2}}^{i_{1}}}+\frac{t_{j_{1} r}^{\phi} c_{r}^{i_{2}}}{c_{j_{1}}^{i_{2}}}+\frac{t_{j_{1} r}^{\phi} c_{r}^{i_{1}}}{c_{j_{1}}^{i_{1}}}\right) \\
& \left.+\frac{1}{2} \sum_{q r}\left(\frac{V_{i_{2} r j_{2} q} c_{r}^{q}}{c_{j_{2}}^{i_{2}}}+\frac{V_{i_{1} r j_{2} q} c_{r}^{q}}{c_{j_{2}}^{i_{1}}}+\frac{V_{i_{2} r j_{1} q} c_{r}^{q}}{c_{j_{1}}^{i_{2}}}+\frac{V_{i_{1} r j_{1} q} c_{r}^{q}}{c_{j_{1}}^{i_{1}}}\right)\right]
\end{aligned}
$$

For $\left\langle\phi_{j_{1} j_{2}}^{i_{1} i_{2}}\left|\hat{H}^{\phi} T^{3}\right| \phi\right\rangle$, all the terms destroying a pair contribute:

$$
\begin{aligned}
& \left\langle\phi_{j_{1} j_{2}}^{i_{1} i_{2}}\left|\hat{H}^{\phi} T^{3}\right| \phi\right\rangle \\
& =\langle\phi| h_{j_{2}} p_{i_{2}} h_{j_{1}} p_{i_{1}}\left(\sum_{m n} t_{m n}^{\phi} h_{n} p_{m}+\frac{1}{2} \sum_{m n q r} V_{m n q r} p_{r}^{\dagger} h_{q} p_{n} p_{m}\right. \\
& \left.+\frac{1}{2} \sum_{m n q r} V_{m n q r} h_{r}^{\dagger} p_{q} h_{n} h_{m}\right) \frac{1}{6} \sum_{k_{1} l_{1} k_{2} l_{2} k_{3} l_{3}} c_{l_{1} l_{2} l_{3}}^{k_{1} k_{2} k_{3}} p_{k_{1}}^{\dagger} h_{l_{1}}^{\dagger} p_{k_{2}}^{\dagger} h_{l_{2}}^{\dagger} p_{k_{3}}^{\dagger} h_{l_{3}}^{\dagger}|\phi\rangle \\
& =\frac{1}{6} \sum_{m n k_{1} l_{1} k_{2} l_{2} k_{3} l_{3}} t_{m n}^{\phi} c_{l_{1} l_{2} l_{3}}^{k_{1} k_{2} k_{3}}\left\langle\phi\left|h_{j_{2}} p_{i_{2}} h_{j_{1}} p_{i_{1}} h_{n} p_{m} p_{k_{1}}^{\dagger} h_{l_{1}}^{\dagger} p_{k_{2}}^{\dagger} h_{l_{2}}^{\dagger} p_{k_{3}}^{\dagger} h_{l_{3}}^{\dagger}\right| \phi\right\rangle \\
& +\frac{1}{12} \sum_{m n q r k_{1} l_{1} k_{2} l_{2} k_{3} l_{3}} V_{m n q r} c_{l_{1} l_{2} l_{3}}^{k_{1} k_{2} k_{3}}\left\langle\phi\left|h_{j_{2}} p_{i_{2}} h_{j_{1}} p_{i_{1}} p_{r}^{\dagger} h_{q} p_{n} p_{m} p_{k_{1}}^{\dagger} h_{l_{1}}^{\dagger} p_{k_{2}}^{\dagger} h_{l_{2}}^{\dagger} p_{k_{3}}^{\dagger} h_{l_{3}}^{\dagger}\right| \phi\right\rangle \\
& +\frac{1}{12} \sum_{m n q r k_{1} l_{1} k_{2} l_{2} k_{3} l_{3}} V_{m n q r} c_{l_{1} l_{2} l_{3}}^{k_{1} k_{2} k_{3}}\left\langle\phi\left|h_{j_{2}} p_{i_{2}} h_{j_{1}} p_{i_{1}} h_{r}^{\dagger} p_{q} h_{n} h_{m} p_{k_{1}}^{\dagger} h_{l_{1}}^{\dagger} p_{k_{2}}^{\dagger} h_{l_{2}}^{\dagger} p_{k_{3}}^{\dagger} h_{l_{3}}^{\dagger}\right| \phi\right\rangle \\
& =\frac{1}{6} \sum_{m n k_{1} l_{1} k_{2} l_{2} k_{3} l_{3}} t_{m n}^{\phi} c_{l_{1} l_{2} l_{3}}^{k_{1} k_{2} k_{3}}\left\langle\phi\left|h_{j_{2}} h_{j_{1}} h_{n} h_{l_{1}}^{\dagger} h_{l_{2}}^{\dagger} h_{l_{3}}^{\dagger} p_{i_{2}} p_{i_{1}} p_{m} p_{k_{1}}^{\dagger} p_{k_{2}}^{\dagger} p_{k_{3}}^{\dagger}\right| \phi\right\rangle \\
& -\frac{1}{12} \sum_{m n q r k_{1} l_{1} k_{2} l_{2} k_{3} l_{3}} V_{m n q r} c_{l_{1} l_{2} l_{3}}^{k_{1} k_{2} k_{3}}\left\langle\phi\left|h_{j_{2}} h_{j_{1}} h_{q} h_{l_{1}}^{\dagger} h_{l_{2}}^{\dagger} h_{l_{3}}^{\dagger} p_{i_{2}} p_{i_{1}} p_{r}^{\dagger} p_{n} p_{m} p_{k_{1}}^{\dagger} p_{k_{2}}^{\dagger} p_{k_{3}}^{\dagger}\right| \phi\right\rangle \\
& +\frac{1}{12} \sum_{m n q r k_{1} l_{1} k_{2} l_{2} k_{3} l_{3}} V_{m n q r} c_{l_{1} l_{2} l_{3}}^{k_{1} k_{2} k_{3}}\left\langle\phi\left|h_{j_{2}} h_{j_{1}} h_{r}^{\dagger} h_{n} h_{m} h_{l_{1}}^{\dagger} h_{l_{2}}^{\dagger} h_{l_{3}}^{\dagger} p_{i_{2}} p_{i_{1}} p_{q} p_{k_{1}}^{\dagger} p_{k_{2}}^{\dagger} p_{k_{3}}^{\dagger}\right| \phi\right\rangle
\end{aligned}
$$


Now, because of the antisymmetry of $c_{l_{1} l_{2} l_{3}}^{k_{1} k_{2} k_{3}}$, we obtain factors of 3 ! for each sum over the indices $\left(k_{1}, k_{2}, k_{3}\right)$ or $\left(l_{1}, l_{2}, l_{3}\right)$ :

$$
\begin{aligned}
\left\langle\phi_{j_{1} j_{2}}^{i_{1} i_{2}}\right| & \hat{H}^{\phi} T^{3}|\phi\rangle \\
& =6 \sum_{m n} t_{m n}^{\phi} c_{n j_{1} j_{2}}^{m i_{1} i_{2}}-\frac{1}{2} \sum_{m n q r k_{1} k_{2} k_{3}} V_{m n q r} c_{q j_{1} j_{2}}^{k_{1} k_{2} k_{3}}\left\langle\phi\left|p_{i_{2}} p_{i_{1}} p_{r}^{\dagger} p_{n} p_{m} p_{k_{1}}^{\dagger} p_{k_{2}}^{\dagger} p_{k_{3}}^{\dagger}\right| \phi\right\rangle \\
& +\frac{1}{2} \sum_{m n q r l_{1} l_{2} l_{3}} V_{m n q r} c_{l_{1} l_{2} l_{3}}^{q i_{1} i_{2}}\left\langle\phi\left|h_{j_{2}} h_{j_{1}} h_{r}^{\dagger} h_{n} h_{m} h_{l_{1}}^{\dagger} h_{l_{2}}^{\dagger} h_{l_{3}}^{\dagger}\right| \phi\right\rangle \\
& =6 \sum_{m n} t_{m n}^{\phi} c_{n j_{1} j_{2}}^{m i_{1} i_{2}}-\frac{1}{2} \sum_{m n q r k_{1} k_{2} k_{3}} V_{m n q r} c_{q j_{1} j_{2}}^{k_{1} k_{2} k_{3}}\left(\delta_{r i_{1}}\left\langle\phi\left|p_{i_{2}} p_{n} p_{m} p_{k_{1}}^{\dagger} p_{k_{2}}^{\dagger} p_{k_{3}}^{\dagger}\right| \phi\right\rangle-\delta_{r i_{2}}\left\langle\phi\left|p_{i_{1}} p_{n} p_{m} p_{k_{1}}^{\dagger} p_{k_{2}}^{\dagger} p_{k_{3}}^{\dagger}\right| \phi\right\rangle\right) \\
& +\frac{1}{2} \sum_{m n q r l_{1} l_{2} l_{3}} V_{m n q r} c_{l_{1} l_{2} l_{3}}^{q i_{1} i_{2}}\left(\delta_{r j_{1}}\left\langle\phi\left|h_{j_{2}} h_{n} h_{m} h_{l_{1}}^{\dagger} h_{l_{2}}^{\dagger} h_{l_{3}}^{\dagger}\right| \phi\right\rangle-\delta_{r j_{2}}\left\langle\phi\left|h_{j_{1}} h_{n} h_{m} h_{l_{1}}^{\dagger} h_{l_{2}}^{\dagger} h_{l_{3}}^{\dagger}\right| \phi\right\rangle\right) \\
& =6 \sum_{m n} t_{m n}^{\phi} c_{n j_{1} j_{2}}^{m i_{1} i_{2}}-3 \sum_{m n q r} V_{m n q r}\left(\delta_{r i_{1}} c_{q j_{1} j_{2}}^{m n i_{2}}-\delta_{r i_{2}} c_{q j_{1} j_{2}}^{m n i_{1}}\right)+3 \sum_{m n q r} V_{m n q r}\left(\delta_{r j_{1}} c_{m n j_{2}}^{q i_{1} i_{2}}-\delta_{r j_{2}} c_{m n j_{1}}^{q i_{1} i_{2}}\right) \\
& =6 \sum_{m n} t_{m n}^{\phi} c_{n j_{1} j_{2}}^{m i_{1} i_{2}}-3 \sum_{m n q}\left(V_{m n q i_{1}} c_{q j_{1} j_{2}}^{m n i_{2}}-V_{m n q i_{2}} c_{q j_{1} j_{2}}^{m n i_{1}}\right)+3 \sum_{m n q}\left(V_{m n q j_{1}} c_{m n j_{2}}^{q i_{1} i_{2}}-V_{m n q j_{2}} c_{m n j_{1}}^{q i_{1} i_{2}}\right)
\end{aligned}
$$

and thus,

$$
\frac{1}{3 !}\left\langle\phi_{j_{1} j_{2}}^{i_{1} i_{2}}\left|\hat{H}^{\phi} T^{3}\right| \phi\right\rangle=\sum_{m n} t_{m n}^{\phi} c_{n j_{1} j_{2}}^{m i_{1} i_{2}}-\frac{1}{2} \sum_{m n q}\left(V_{m n q i_{1}} c_{q j_{1} j_{2}}^{m n i_{2}}-V_{m n q i_{2}} c_{q j_{1} j_{2}}^{m n i_{1}}\right)+\frac{1}{2} \sum_{m n q}\left(V_{m n q j_{1}} c_{m n j_{2}}^{q i_{1} i_{2}}-V_{m n q j_{2}} c_{m n j_{1}}^{q i_{1} i_{2}}\right)
$$

For $\left\langle\phi_{j_{1} j_{2}}^{i_{1} i_{2}}\left|T \hat{H}^{\phi} T^{2}\right| \phi\right\rangle$, all the terms of $H$ destroying on pair contribute:

$$
\begin{aligned}
& \left\langle\phi_{j_{1} j_{2}}^{i_{1} i_{2}}\left|T \hat{H}^{\phi} T^{2}\right| \phi\right\rangle \\
& =\langle\phi| h_{j_{2}} p_{i_{2}} h_{j_{1}} p_{i_{1}} \sum_{k_{1} l_{1}} p_{k_{1}}^{\dagger} h_{l_{1}}^{\dagger} \hat{D}_{l_{1}}^{k_{1}}\left(\sum_{m n} t_{m n}^{\phi} h_{n} p_{m}+\frac{1}{2} \sum_{m n q r} V_{m n q r} p_{r}^{\dagger} h_{q} p_{n} p_{m}\right. \\
& \left.+\frac{1}{2} \sum_{m n q r} V_{m n q r} h_{r}^{\dagger} p_{q} h_{n} h_{m}\right) \frac{1}{2} \sum_{k_{2} l_{2} k_{3} l_{3}} c_{l_{2} l_{3}}^{k_{2} k_{3}} p_{k_{2}}^{\dagger} h_{l_{2}}^{\dagger} p_{k_{3}}^{\dagger} h_{l_{3}}^{\dagger}|\phi\rangle \\
& =\frac{1}{2} \sum_{k_{1} l_{1} k_{2} l_{2} k_{3} l_{3} m n} t_{m n}^{\phi} c_{l_{2} l_{3}}^{k_{2} k_{3}}\left\langle\phi\left|h_{j_{2}} p_{i_{2}} h_{j_{1}} p_{i_{1}} p_{k_{1}}^{\dagger} h_{l_{1}}^{\dagger} \hat{D}_{l_{1}}^{k_{1}} h_{n} p_{m} p_{k_{2}}^{\dagger} h_{l_{2}}^{\dagger} p_{k_{3}}^{\dagger} h_{l_{3}}^{\dagger}\right| \phi\right\rangle \\
& +\frac{1}{4} \sum_{k_{1} l_{1} k_{2} l_{2} k_{3} l_{3} m n q r} V_{m n q r} c_{l_{2} l_{3}}^{k_{2} k_{3}}\left\langle\phi\left|h_{j_{2}} p_{i_{2}} h_{j_{1}} p_{i_{1}} p_{k_{1}}^{\dagger} h_{l_{1}}^{\dagger} \hat{D}_{l_{1}}^{k_{1}} p_{r}^{\dagger} h_{q} p_{n} p_{m} p_{k_{2}}^{\dagger} h_{l_{2}}^{\dagger} p_{k_{3}}^{\dagger} h_{l_{3}}^{\dagger}\right| \phi\right\rangle \\
& +\frac{1}{4} \sum_{k_{1} l_{1} k_{2} l_{2} k_{3} l_{3} m n q r} V_{m n q r} c_{l_{2} l_{3}}^{k_{2} k_{3}}\left\langle\phi\left|h_{j_{2}} p_{i_{2}} h_{j_{1}} p_{i_{1}} p_{k_{1}}^{\dagger} h_{l_{1}}^{\dagger} \hat{D}_{l_{1}}^{k_{1}} h_{r}^{\dagger} p_{q} h_{n} h_{m} p_{k_{2}}^{\dagger} h_{l_{2}}^{\dagger} p_{k_{3}}^{\dagger} h_{l_{3}}^{\dagger}\right| \phi\right\rangle \\
& =\frac{1}{2} \sum_{k_{1} l_{1} k_{2} l_{2} k_{3} l_{3} m n} t_{m n}^{\phi} c_{l_{2} l_{3}}^{k_{2} k_{3}}\left\langle\phi\left|h_{j_{2}} p_{i_{2}} h_{j_{1}} p_{i_{1}} p_{k_{1}}^{\dagger} h_{l_{1}}^{\dagger} \hat{D}_{l_{1}}^{k_{1}} p_{m} p_{k_{2}}^{\dagger} p_{k_{3}}^{\dagger} h_{n} h_{l_{2}}^{\dagger} h_{l_{3}}^{\dagger}\right| \phi\right\rangle \\
& -\frac{1}{4} \sum_{k_{1} l_{1} k_{2} l_{2} k_{3} l_{3} m n q r} V_{m n q r} c_{l_{2} l_{3}}^{k_{2} k_{3}}\left\langle\phi\left|h_{j_{2}} p_{i_{2}} h_{j_{1}} p_{i_{1}} p_{k_{1}}^{\dagger} h_{l_{1}}^{\dagger} \hat{D}_{l_{1}}^{k_{1}} p_{r}^{\dagger} p_{n} p_{m} p_{k_{2}}^{\dagger} p_{k_{3}}^{\dagger} h_{q} h_{l_{2}}^{\dagger} h_{l_{3}}^{\dagger}\right| \phi\right\rangle \\
& +\frac{1}{4} \sum_{k_{1} l_{1} k_{2} l_{2} k_{3} l_{3} m n q r} V_{m n q r} c_{l_{2} l_{3}}^{k_{2} k_{3}}\left\langle\phi\left|h_{j_{2}} p_{i_{2}} h_{j_{1}} p_{i_{1}} p_{k_{1}}^{\dagger} h_{l_{1}}^{\dagger} \hat{D}_{l_{1}}^{k_{1}} p_{q} p_{k_{2}}^{\dagger} p_{k_{3}}^{\dagger} h_{r}^{\dagger} h_{n} h_{m} h_{l_{2}}^{\dagger} h_{l_{3}}^{\dagger}\right| \phi\right\rangle
\end{aligned}
$$


$\left\langle\phi_{j_{1} j_{2}}^{i_{1} i_{2}}\left|T \hat{H}^{\phi} T^{2}\right| \phi\right\rangle$

$$
\begin{aligned}
& =\frac{1}{2} \sum_{k_{1} l_{1} k_{2} l_{2} k_{3} l_{3} m n} t_{m n}^{\phi} c_{l_{2} l_{3}}^{k_{2} k_{3}}\left\langle\phi\left|h_{j_{2}} p_{i_{2}} h_{j_{1}} p_{i_{1}} p_{k_{1}}^{\dagger} h_{l_{1}}^{\dagger} \hat{D}_{l_{1}}^{k_{1}}\left(\delta_{m k_{2}} p_{k_{3}}^{\dagger}-\delta_{m k_{3}} p_{k_{2}}^{\dagger}\right)\left(\delta_{n l_{2}} h_{l_{3}}^{\dagger}-\delta_{n l_{3}} h_{l_{2}}^{\dagger}\right)\right| \phi\right\rangle \\
& -\frac{1}{4} \sum_{k_{1} l_{1} k_{2} l_{2} k_{3} l_{3} m n q r} V_{m n q r} c_{l_{2} l_{3}}^{k_{2} k_{3}}\left(\delta_{m k_{2}} \delta_{n k_{3}}-\delta_{m k_{3}} \delta_{n k_{2}}\right)\left\langle\phi\left|h_{j_{2}} p_{i_{2}} h_{j_{1}} p_{i_{1}} p_{k_{1}}^{\dagger} h_{l_{1}}^{\dagger} \hat{D}_{l_{1}}^{k_{1}} p_{r}^{\dagger}\left(\delta_{q l_{2}} h_{l_{3}}^{\dagger}-\delta_{q l_{3}} h_{l_{2}}^{\dagger}\right)\right| \phi\right\rangle \\
& +\frac{1}{4} \sum_{k_{1} l_{1} k_{2} l_{2} k_{3} l_{3} m n q r} V_{m n q r} c_{l_{2} l_{3}}^{k_{2} k_{3}}\left(\delta_{m l_{2}} \delta_{n l_{3}}-\delta_{m l_{3}} \delta_{n l_{2}}\right)\left\langle\phi\left|h_{j_{2}} p_{i_{2}} h_{j_{1}} p_{i_{1}} p_{k_{1}}^{\dagger} h_{l_{1}}^{\dagger} \hat{D}_{l_{1}}^{k_{1}}\left(\delta_{q k_{2}} p_{k_{3}}^{\dagger}-\delta_{q k_{3}} p_{k_{2}}^{\dagger}\right) h_{r}^{\dagger}\right| \phi\right\rangle \\
& =\frac{1}{2} \sum_{k_{1} l_{1} k_{2} l_{2} k_{3} l_{3} m n} t_{m n}^{\phi} c_{l_{2} l_{3}}^{k_{2} k_{3}}\left(\delta_{m k_{2}} \delta_{n l_{2}} \frac{c_{l_{1} l_{3}}^{k_{1} k_{3}}}{c_{l_{3}}^{k_{3}}}\left\langle\phi\left|h_{j_{2}} p_{i_{2}} h_{j_{1}} p_{i_{1}} p_{k_{1}}^{\dagger} h_{l_{1}}^{\dagger} p_{k_{3}}^{\dagger} h_{l_{3}}^{\dagger}\right| \phi\right\rangle\right. \\
& -\delta_{m k_{2}} \delta_{n l_{3}} \frac{c_{l_{1} l_{2}}^{k_{1} k_{3}}}{c_{l_{2}}^{k_{3}}}\left\langle\phi\left|h_{j_{2}} p_{i_{2}} h_{j_{1}} p_{i_{1}} p_{k_{1}}^{\dagger} h_{l_{1}}^{\dagger} p_{k_{3}}^{\dagger} h_{l_{2}}^{\dagger}\right| \phi\right\rangle \\
& -\delta_{m k_{3}} \delta_{n l_{2}} \frac{c_{l_{1} l_{2}}^{k_{1} k_{2}}}{c_{l_{3}}^{k_{2}}}\left\langle\phi\left|h_{j_{2}} p_{i_{2}} h_{j_{1}} p_{i_{1}} p_{k_{1}}^{\dagger} h_{l_{1}}^{\dagger} p_{k_{2}}^{\dagger} h_{l_{3}}^{\dagger}\right| \phi\right\rangle \\
& \left.+\delta_{m k_{3}} \delta_{n l_{3}} \frac{c_{l_{1} l_{2}}^{k_{1} k_{2}}}{c_{l_{2}}^{k_{2}}}\left\langle\phi\left|h_{j_{2}} p_{i_{2}} h_{j_{1}} p_{i_{1}} p_{k_{1}}^{\dagger} h_{l_{1}}^{\dagger} p_{k_{2}}^{\dagger} h_{l_{2}}^{\dagger}\right| \phi\right\rangle\right) \\
& -\frac{1}{2} \sum_{k_{1} l_{1} l_{2} l_{3} m n q r} V_{m n q r} c_{l_{2} l_{3}}^{m n}\left(\delta_{q l_{2}} \frac{c_{l_{1} l_{3}}^{k_{1} r}}{c_{l_{3}}^{r}}\left\langle\phi\left|h_{j_{2}} p_{i_{2}} h_{j_{1}} p_{i_{1}} p_{k_{1}}^{\dagger} h_{l_{1}}^{\dagger} p_{r}^{\dagger} h_{l_{3}}^{\dagger}\right| \phi\right\rangle-\delta_{q l_{3}} \frac{c_{l_{1} l_{2}}^{k_{1} r}}{c_{l_{2}}^{r}}\left\langle\phi\left|h_{j_{2}} p_{i_{2}} h_{j_{1}} p_{i_{1}} p_{k_{1}}^{\dagger} h_{l_{1}}^{\dagger} p_{r}^{\dagger} h_{l_{2}}^{\dagger}\right| \phi\right\rangle\right) \\
& +\frac{1}{2} \sum_{k_{1} l_{1} k_{2} k_{3} m n q r} V_{m n q r} c_{m n}^{k_{2} k_{3}}\left(\delta_{q k_{2}} \frac{c_{l_{1} r}^{k_{1} k_{3}}}{c_{r}^{k_{3}}}\left\langle\phi\left|h_{j_{2}} p_{i_{2}} h_{j_{1}} p_{i_{1}} p_{k_{1}}^{\dagger} h_{l_{1}}^{\dagger} p_{k_{3}}^{\dagger} h_{r}^{\dagger}\right| \phi\right\rangle-\delta_{q k_{3}} \frac{c_{l_{1} r}^{k_{1} k_{2}}}{c_{r}^{k_{2}}}\left\langle\phi\left|h_{j_{2}} p_{i_{2}} h_{j_{1}} p_{i_{1}} p_{k_{1}}^{\dagger} h_{l_{1}}^{\dagger} p_{k_{2}}^{\dagger} h_{r}^{\dagger}\right| \phi\right\rangle\right) \\
& =\frac{1}{2} \sum_{k_{1} l_{1} k_{2} l_{2} k_{3} l_{3} m n} t_{m n}^{\phi} c_{l_{2} l_{3}}^{k_{2} k_{3}}\left(\delta_{m k_{2}} \delta_{n l_{2}} \frac{c_{l_{1} l_{3}}^{k_{1} k_{3}}}{c_{l_{3}}^{k_{3}}}\left\langle\phi\left|h_{j_{2}} h_{j_{1}} h_{l_{1}}^{\dagger} h_{l_{3}}^{\dagger} p_{i_{2}} p_{i_{1}} p_{k_{1}}^{\dagger} p_{k_{3}}^{\dagger}\right| \phi\right\rangle\right. \\
& -\delta_{m k_{2}} \delta_{n l_{3}} \frac{c_{l_{1} l_{2}}^{k_{1} k_{3}}}{c_{l_{2}}^{k_{3}}}\left\langle\phi\left|h_{j_{2}} h_{j_{1}} h_{l_{1}}^{\dagger} h_{l_{2}}^{\dagger} p_{i_{2}} p_{i_{1}} p_{k_{1}}^{\dagger} p_{k_{3}}^{\dagger}\right| \phi\right\rangle \\
& -\delta_{m k_{3}} \delta_{n l_{2}} \frac{c_{l_{1} l_{3}}^{k_{1} k_{2}}}{c_{l_{3}}^{k_{2}}}\left\langle\phi\left|h_{j_{2}} h_{j_{1}} h_{l_{1}}^{\dagger} h_{l_{3}}^{\dagger} p_{i_{2}} p_{i_{1}} p_{k_{1}}^{\dagger} p_{k_{2}}^{\dagger}\right| \phi\right\rangle \\
& \left.+\delta_{m k_{3}} \delta_{n l_{3}} \frac{c_{l_{1} l_{2}}^{k_{1} k_{2}}}{c_{l_{2}}^{k_{2}}}\left\langle\phi\left|h_{j_{2}} h_{j_{1}} h_{l_{1}}^{\dagger} h_{l_{2}}^{\dagger} p_{i_{2}} p_{i_{1}} p_{k_{1}}^{\dagger} p_{k_{2}}^{\dagger}\right| \phi\right\rangle\right) \\
& -\frac{1}{2} \sum_{k_{1} l_{1} l_{2} l_{3} m n q r} V_{m n q r} c_{l_{2} l_{3}}^{m n}\left(\delta_{q l_{2}} \frac{c_{l_{1} l_{3}}^{k_{1} r}}{c_{l_{3}}^{r}}\left\langle\phi\left|h_{j_{2}} h_{j_{1}} h_{l_{1}}^{\dagger} h_{l_{3}}^{\dagger} p_{i_{2}} p_{i_{1}} p_{k_{1}}^{\dagger} p_{r}^{\dagger}\right| \phi\right\rangle-\delta_{q l_{3}} \frac{c_{l_{1} l_{2}}^{k_{1} r}}{c_{l_{2}}^{r}}\left\langle\phi\left|h_{j_{2}} h_{j_{1}} h_{l_{1}}^{\dagger} h_{l_{2}}^{\dagger} p_{i_{2}} p_{i_{1}} p_{k_{1}}^{\dagger} p_{r}^{\dagger}\right| \phi\right\rangle\right) \\
& +\frac{1}{2} \sum_{k_{1} l_{1} k_{2} k_{3} m n q r} V_{m n q r} c_{m n}^{k_{2} k_{3}}\left(\delta_{q k_{2}} \frac{c_{l_{1} r}^{k_{1} k_{3}}}{c_{r}^{k_{3}}}\left\langle\phi\left|h_{j_{2}} h_{j_{1}} h_{l_{1}}^{\dagger} h_{r}^{\dagger} p_{i_{2}} p_{i_{1}} p_{k_{1}}^{\dagger} p_{k_{3}}^{\dagger}\right| \phi\right\rangle-\delta_{q k_{3}} \frac{c_{l_{1} r}^{k_{1} k_{2}}}{c_{r}^{k_{2}}}\left\langle\phi\left|h_{j_{2}} h_{j_{1}} h_{l_{1}}^{\dagger} h_{r}^{\dagger} p_{i_{2}} p_{i_{1}} p_{k_{1}}^{\dagger} p_{k_{2}}^{\dagger}\right| \phi\right\rangle\right)
\end{aligned}
$$




$$
\begin{aligned}
& \left\langle\phi_{j_{1} j_{2}}^{i_{1} i_{2}}\left|T \hat{H}^{\phi} T^{2}\right| \phi\right\rangle \\
& =\frac{1}{2} \sum_{k_{1} l_{1} k_{2} l_{2} k_{3} l_{3} m n} t_{m n}^{\phi} c_{l_{2} l_{3}}^{k_{2} k_{3}}\left(\frac{c_{l_{1} l_{3}}^{k_{1} k_{3}}}{c_{l_{3}}^{k_{3}}} \delta_{m k_{2}} \delta_{n l_{2}}\left(\delta_{l_{1} j_{1}} \delta_{l_{3} j_{2}}-\delta_{l_{1} j_{2}} \delta_{l_{3} j_{1}}\right)\left(\delta_{k_{1} i_{1}} \delta_{k_{3} i_{2}}-\delta_{k_{1} i_{2}} \delta_{k_{3} i_{1}}\right)\right. \\
& -\frac{c_{l_{1} l_{2}}^{k_{1} k_{3}}}{c_{l_{2}}^{k_{3}}} \delta_{m k_{2}} \delta_{n l_{3}}\left(\delta_{l_{1} j_{1}} \delta_{l_{2} j_{2}}-\delta_{l_{1} j_{2}} \delta_{l_{2} j_{1}}\right)\left(\delta_{k_{1} i_{1}} \delta_{k_{3} i_{2}}-\delta_{k_{1} i_{2}} \delta_{k_{3} i_{1}}\right) \\
& -\frac{c_{l_{1} l_{3}}^{k_{1} k_{2}}}{c_{l_{3}}^{k_{2}}} \delta_{m k_{3}} \delta_{n l_{2}}\left(\delta_{l_{1} j_{1}} \delta_{l_{3} j_{2}}-\delta_{l_{1} j_{2}} \delta_{l_{3} j_{1}}\right)\left(\delta_{k_{1} i_{1}} \delta_{k_{2} i_{2}}-\delta_{k_{1} i_{2}} \delta_{k_{2} i_{1}}\right) \\
& \left.+\frac{c_{l_{1} l_{2}}^{k_{1} k_{2}}}{c_{l_{2}}^{k_{2}}} \delta_{m k_{3}} \delta_{n l_{3}}\left(\delta_{l_{1} j_{1}} \delta_{l_{2} j_{2}}-\delta_{l_{1} j_{2}} \delta_{l_{2} j_{1}}\right)\left(\delta_{k_{1} i_{1}} \delta_{k_{2} i_{2}}-\delta_{k_{1} i_{2}} \delta_{k_{2} i_{1}}\right)\right) \\
& -\frac{1}{2} \sum_{k_{1} l_{1} l_{2} l_{3} m n q r} V_{m n q r} c_{l_{2} l_{3}}^{m n}\left(\delta_{q l_{2}} \frac{c_{l_{1} l_{3}}^{k_{1} r}}{c_{l_{3}}^{r}}\left(\delta_{l_{1} j_{1}} \delta_{l_{3} j_{2}}-\delta_{l_{1} j_{2}} \delta_{l_{3} j_{1}}\right)\left(\delta_{k_{1} i_{1}} \delta_{r i_{2}}-\delta_{k_{1} i_{2}} \delta_{r i_{1}}\right)\right. \\
& \left.-\delta_{q l_{3}} \frac{c_{l_{1} l_{2}}^{k_{1} r}}{c_{l_{2}}^{r}}\left(\delta_{l_{1} j_{1}} \delta_{l_{2} j_{2}}-\delta_{l_{1} j_{2}} \delta_{l_{2} j_{1}}\right)\left(\delta_{k_{1} i_{1}} \delta_{r i_{2}}-\delta_{k_{1} i_{2}} \delta_{r i_{1}}\right)\right) \\
& +\frac{1}{2} \sum_{k_{1} l_{1} k_{2} k_{3} m n q r} V_{m n q r} c_{m n}^{k_{2} k_{3}}\left(\delta_{q k_{2}} \frac{c_{l_{1} r}^{k_{1} k_{3}}}{c_{r}^{k_{3}}}\left(\delta_{l_{1} j_{1}} \delta_{r j_{2}}-\delta_{l_{1} j_{2}} \delta_{r j_{1}}\right)\left(\delta_{k_{1} i_{1}} \delta_{k_{3} i_{2}}-\delta_{k_{1} i_{2}} \delta_{k_{3} i_{1}}\right)\right. \\
& \left.-\delta_{q k_{3}} \frac{c_{l_{1} r}^{k_{1} k_{2}}}{c_{r}^{k_{2}}}\left(\delta_{l_{1} j_{1}} \delta_{r j_{2}}-\delta_{l_{1} j_{2}} \delta_{r j_{1}}\right)\left(\delta_{k_{1} i_{1}} \delta_{k_{2} i_{2}}-\delta_{k_{1} i_{2}} \delta_{k_{2} i_{1}}\right)\right) \\
& =\frac{1}{2} \sum_{m n} t_{m n}^{\phi}\left[c_{n j_{2}}^{m i_{2}} \frac{c_{j_{1} j_{2}}^{i_{1} i_{2}}}{c_{j_{2}}^{i_{2}}}-c_{n j_{2}}^{m i_{1}} \frac{c_{j_{1} j_{2}}^{i_{2} i_{1}}}{c_{j_{2}}^{i_{1}}}-c_{n j_{1}}^{m i_{2}} \frac{c_{j_{2} j_{1}}^{i_{1} i_{2}}}{c_{j_{1}}^{i_{2}}}+c_{n j_{1}}^{m i_{1}} \frac{c_{j_{2} j_{1}}^{i_{2} i_{1}}}{c_{j_{1}}^{i_{1}}}\right. \\
& -\left(c_{j_{2} n}^{m i_{2}} \frac{c_{j_{1} j_{2}}^{i_{1} i_{2}}}{c_{j_{2}}^{i_{2}}}-c_{j_{2} n}^{m i_{1}} \frac{c_{j_{j_{2}}}^{i_{2} i_{1}}}{c_{j_{2}}^{i_{1}}}-c_{j_{1} n}^{m i_{2}} \frac{c_{j_{2} j_{1}}^{i_{1} i_{2}}}{c_{j_{1}}^{i_{2}}}+c_{j_{1} n}^{m i_{1}} \frac{c_{j_{2} j_{1}}^{i_{2} i_{1}}}{c_{j_{1}}^{i_{1}}}\right) \\
& -\left(c_{n j_{2}}^{i_{2} m} \frac{c_{j_{1} j_{2}}^{i_{1} i_{2}}}{c_{j_{2}}^{i_{2}}}-c_{n j_{2}}^{i_{1} m} \frac{c_{j_{1} j_{2}}^{i_{2} i_{1}}}{c_{j_{2}}^{i_{1}}}-c_{n j_{1}}^{i_{2} m} \frac{c_{j_{2} j_{1}}^{i_{1} i_{2}}}{c_{j_{1}}^{i_{2}}}+c_{n j_{1}}^{i_{1} m} \frac{c_{j_{2} j_{1}}^{i_{2} i_{1}}}{c_{j_{1}}^{i_{1}}}\right) \\
& \left.+c_{j_{2} n}^{i_{2} m} \frac{c_{j_{1} j_{2}}^{i_{1} i_{2}}}{c_{j_{2}}^{i_{2}}}-c_{j_{2} n}^{i_{1} m} \frac{c_{j_{1} j_{2}}^{i_{2} i_{1}}}{c_{j_{2}}^{i_{1}}}-c_{j_{1} n}^{i_{2} m} \frac{c_{j_{2} j_{1}}^{i_{1} i_{2}}}{c_{j_{1}}^{i_{2}}}+c_{j_{1} n}^{i_{1} m} \frac{c_{j_{2} j_{1}}^{i_{2} i_{1}}}{c_{j_{1}}^{i_{1}}}\right] \\
& -\frac{1}{2} \sum_{m n q}\left[V_{m n q i_{2}} c_{q j_{2}}^{m n} \frac{c_{j_{1} j_{2}}^{i_{1} i_{2}}}{c_{j_{2}}^{i_{2}}}-V_{m n q i_{1}} c_{q j_{2}}^{m n} \frac{c_{j_{1} j_{2}}^{i_{2} i_{1}}}{c_{j_{2}}^{i_{1}}}-V_{m n q i_{2}} c_{q j_{1}}^{m n} \frac{c_{j_{2} j_{1}}^{i_{1} i_{2}}}{c_{j_{1}}^{i_{2}}}+V_{m n q i_{1}} c_{q j_{1}}^{m n} \frac{c_{j_{2} j_{1}}^{i_{2} i_{1}}}{c_{j_{1}}^{i_{1}}}\right. \\
& \left.-\left(V_{m n q i_{2}} c_{j_{2} q}^{m n} \frac{c_{j_{1} j_{2}}^{i_{1} i_{2}}}{c_{j_{2}}^{i_{2}}}-V_{m n q i_{1}} c_{j_{2} q}^{m n} \frac{c_{j_{1} j_{2}}^{i_{2} i_{1}}}{c_{j_{2}}^{i_{1}}}-V_{m n q i_{2}} c_{j_{1} q}^{m n} \frac{c_{j_{2} j_{1}}^{i_{1} i_{2}}}{c_{j_{1}}^{i_{2}}}+V_{m n q i_{1}} c_{j_{1} q}^{m n} \frac{c_{j_{2} j_{1}}^{i_{2} i_{1}}}{c_{j_{1}}^{i_{1}}}\right)\right] \\
& +\frac{1}{2} \sum_{m n q}\left[V_{m n q j_{2}} c_{m n}^{q i_{2}} \frac{c_{j_{1} j_{2}}^{i_{1} i_{2}}}{c_{j_{2}}^{i_{2}}}-V_{m n q j_{2}} c_{m n}^{q i_{1}} \frac{c_{j_{1} j_{2}}^{i_{2} i_{1}}}{c_{j_{2}}^{i_{1}}}-V_{m n q j_{1}} c_{m n}^{q i_{2}} \frac{c_{j_{2} j_{1}}^{i_{1} i_{2}}}{c_{j_{1}}^{i_{2}}}+V_{m n q j_{1}} m_{m n}^{q i_{1}} \frac{c_{j_{2} j_{1}}^{i_{2} i_{1}}}{c_{j_{1}}^{i_{1}}}\right. \\
& \left.-\left(V_{m n q j_{2}} c_{m n}^{i_{2} q} \frac{c_{j_{1} j_{2}}^{i_{1} i_{2}}}{c_{j_{2}}^{i_{2}}}-V_{m n q j_{2}} c_{m n}^{i_{1} q} \frac{c_{j_{1} j_{2}}^{i_{2} i_{1}}}{c_{j_{2}}^{i_{1}}}-V_{m n q j_{1}} c_{m n}^{i_{2} q} \frac{c_{j_{2} j_{1}}^{i_{1} i_{2}}}{c_{j_{1}}^{i_{2}}}+V_{m n q j_{1}} c_{m n}^{i_{1} q} \frac{c_{j_{2} j_{1}}^{i_{2} i_{1}}}{c_{j_{1}}^{i_{1}}}\right)\right]
\end{aligned}
$$


and again, using the antisymmetry of $c_{j_{1} j_{2}}^{i_{1} i_{2}}$,

$$
\begin{aligned}
-\frac{1}{2}\left\langle\phi_{j_{1} j_{2}}^{i_{1} i_{2}}\left|T \hat{H}^{\phi} T^{2}\right| \phi\right\rangle & =c_{j_{1} j_{2}}^{i_{1} i_{2}}\left[-\sum_{m n} t_{m n}^{\phi}\left(\frac{c_{n j_{2}}^{m i_{2}}}{c_{j_{2}}^{i_{2}}}+\frac{c_{n j_{2}}^{m i_{1}}}{c_{j_{2}}^{i_{1}}}+\frac{c_{n j_{1}}^{m i_{2}}}{c_{j_{1}}^{i_{2}}}+\frac{c_{n j_{1}}^{m i_{1}}}{c_{j_{1}}^{i_{1}}}\right)\right. \\
& +\frac{1}{2} \sum_{m n q}\left(\frac{V_{m n q i_{2}} c_{q j_{2}}^{m n}}{c_{j_{2}}^{i_{2}}}+\frac{V_{m n q i_{1}} c_{q j_{2}}^{m n}}{c_{j_{2}}^{i_{1}}}+\frac{V_{m n q i_{2}} c_{q j_{1}}^{m n}}{c_{j_{1}}^{i_{2}}}+\frac{V_{m n q i_{1}} c_{q j_{1}}^{m n}}{c_{j_{1}}^{i_{1}}}\right) \\
& \left.-\frac{1}{2} \sum_{m n q}\left(\frac{V_{m n q j_{2}} c_{m n}^{q i_{2}}}{c_{j_{2}}^{i_{2}}}+\frac{V_{m n q j_{2}} c_{m n}^{q i_{1}}}{c_{j_{2}}^{i_{1}}}+\frac{V_{m n q j_{1}} c_{m n}^{q i_{2}}}{c_{j_{1}}^{i_{2}}}+\frac{V_{m n q j_{1}} c_{m n}^{q i_{1}}}{c_{j_{1}}^{i_{1}}}\right)\right]
\end{aligned}
$$

For $\left\langle\phi_{j_{1} j_{2}}^{i_{1} i_{2}}\left|T^{2} \hat{H}^{\phi} T\right| \phi\right\rangle$ we have

$$
\begin{aligned}
\left\langle\phi_{j_{1} j_{2}}^{i_{1} i_{2}}\left|T^{2} \hat{H}^{\phi} T\right| \phi\right\rangle & =\left\langle\phi_{j_{1} j_{2}}^{i_{1} i_{2}}\left|T^{2}\right| \phi\right\rangle\left\langle\phi\left|\hat{H}^{\phi} T\right| \phi\right\rangle \\
& =\frac{1}{2} \sum_{k_{1} l_{1} k_{2} l_{2}} c_{l_{1} l_{2}}^{k_{1} k_{2}}\left\langle\phi\left|h_{j_{2}} p_{i_{2}} h_{j_{1}} p_{i_{1}} p_{k_{1}}^{\dagger} h_{l_{1}}^{\dagger} p_{k_{2}}^{\dagger} h_{l_{2}}^{\dagger}\right| \phi\right\rangle \sum_{m n k_{3} l_{3}} t_{m n}^{\phi} c_{l_{3}}^{k_{3}}\left\langle\phi\left|h_{n} p_{m} p_{k_{3}}^{\dagger} h_{l_{3}}^{\dagger}\right| \phi\right\rangle \\
& =2 c_{j_{1} j_{2}}^{i_{1} i_{2}} \sum_{m n} t_{m n}^{\phi} c_{n}^{m}
\end{aligned}
$$

and thus,

$$
\frac{1}{2}\left\langle\phi_{j_{1} j_{2}}^{i_{1} i_{2}}\left|T^{2} \hat{H}^{\phi} T\right| \phi\right\rangle=c_{j_{1} j_{2}}^{i_{1} i_{2}} \sum_{m n} t_{m n}^{\phi} c_{n}^{m}
$$

$$
\begin{aligned}
\left\langle\phi_{j_{1} j_{2}}^{i_{1} i_{2}}\left|\hat{H}^{\phi} T^{4}\right| \phi\right\rangle & \\
& =\frac{1}{4(4 !)} \sum_{m n q r k_{1} l_{1} k_{2} l_{2} k_{3} l_{3} k_{4} l_{4}} V_{m n q r} c_{l_{1} l_{2} l_{3} l_{4}}^{k_{1} k_{2} k_{3} k_{4}}\left\langle\phi\left|h_{j_{2}} p_{i_{2}} h_{j_{1}} p_{i_{1}} h_{r} h_{q} p_{n} p_{m} p_{k_{1}}^{\dagger} h_{l_{1}}^{\dagger} p_{k_{2}}^{\dagger} h_{l_{2}}^{\dagger} p_{k_{3}}^{\dagger} h_{l_{3}}^{\dagger} p_{k_{4}}^{\dagger} h_{l_{4}}^{\dagger}\right| \phi\right\rangle \\
& =-\frac{1}{4(4 !)} \sum_{m n q r k_{1} l_{1} k_{2} l_{2} k_{3} l_{3} k_{4} l_{4}} V_{m n q r} c_{l_{1} l_{2} l_{3} l_{4}}^{k_{1} k_{2} k_{3} k_{4}}\left\langle\phi\left|p_{i_{2}} p_{i_{1}} p_{n} p_{m} p_{k_{1}}^{\dagger} p_{k_{2}}^{\dagger} p_{k_{3}}^{\dagger} p_{k_{4}}^{\dagger} h_{j_{2}} h_{j_{1}} h_{r} h_{q} h_{l_{1}}^{\dagger} h_{l_{2}}^{\dagger} h_{l_{3}}^{\dagger} h_{l_{4}}^{\dagger}\right| \phi\right\rangle \\
& =-\frac{4 !}{4} \sum_{m n q r} V_{m n q r} c_{q r j_{1} j_{2}}^{m n i_{1} i_{2}}
\end{aligned}
$$

and

$$
\frac{1}{4 !}\left\langle\phi_{j_{1} j_{2}}^{i_{1} i_{2}}\left|\hat{H}^{\phi} T^{4}\right| \phi\right\rangle=-\frac{1}{4} \sum_{m n q r} V_{m n q r} c_{q r j_{1} j_{2}}^{m n i_{1} i_{2}}
$$




$$
\begin{aligned}
\left\langle\phi_{j_{1} j_{2}}^{i_{1} i_{2}}\right| & T H^{\phi} T^{3}|\phi\rangle \\
= & \left\langle\phi\left|h_{j_{2}} p_{i_{2}} h_{j_{1}} p_{i_{1}} \sum_{k_{1} l_{1}} p_{k_{1}}^{\dagger} h_{l_{1}}^{\dagger} \hat{D}_{l_{1}}^{k_{1}}\left(\frac{1}{4}\right) \sum_{m n q r} V_{m n q r} h_{r} h_{q} p_{n} p_{m}\left(\frac{1}{3 !}\right) \sum_{k_{2} l_{2} k_{3} l_{3} k_{4} l_{4}} c_{l_{2} l_{3} l_{4}}^{k_{2} k_{3} k_{4}} p_{k_{2}}^{\dagger} h_{l_{2}}^{\dagger} p_{k_{3}}^{\dagger} h_{l_{3}}^{\dagger} p_{k_{4}}^{\dagger} h_{l_{4}}^{\dagger}\right| \phi\right\rangle \\
= & \frac{1}{24} \sum_{m n q r k_{1} l_{1} k_{2} l_{2} k_{3} l_{3} k_{4} l_{4}} V_{m n q r} c_{l_{2} l_{3} l_{4}}^{k_{2} k_{3} k_{4}}\left\langle\phi\left|h_{j_{2}} p_{i_{2}} h_{j_{1}} p_{i_{1}} p_{k_{1}}^{\dagger} h_{l_{1}}^{\dagger} \hat{D}_{l_{1}}^{k_{1}} h_{r} h_{q} p_{n} p_{m} p_{k_{2}}^{\dagger} h_{l_{2}}^{\dagger} p_{k_{3}}^{\dagger} h_{l_{3}}^{\dagger} p_{k_{4}}^{\dagger} h_{l_{4}}^{\dagger}\right| \phi\right\rangle \\
= & -\frac{1}{24} \sum_{m n q r k_{1} l_{1} k_{2} l_{2} k_{3} l_{3} k_{4} l_{4}} V_{m n q r} c_{l_{2} l_{3} l_{4}}^{k_{2} k_{3} k_{4}}\left\langle\phi\left|h_{j_{2}} p_{i_{2}} h_{j_{1}} p_{i_{1}} p_{k_{1}}^{\dagger} h_{l_{1}}^{\dagger} \hat{D}_{l_{1}}^{k_{1}} p_{n} p_{m} p_{k_{2}}^{\dagger} p_{k_{3}}^{\dagger} p_{k_{4}}^{\dagger} h_{r} h_{q} h_{l_{2}}^{\dagger} h_{l_{3}}^{\dagger} h_{l_{4}}^{\dagger}\right| \phi\right\rangle \\
= & -\frac{1}{24} \sum_{m n q r k_{1} l_{1} k_{2} l_{2} k_{3} l_{3} k_{4} l_{4}} V_{m n q r} c_{l_{2} l_{3} l_{4}}^{k_{2} k_{3} k_{4}}\langle\phi| h_{j_{2}} p_{i_{2}} h_{j_{1}} p_{i_{1}} p_{k_{1}}^{\dagger} h_{l_{1}}^{\dagger} \hat{D}_{l_{1}}^{k_{1}} \\
& \times\left(\delta_{k_{2} m} \delta_{k_{3} n} p_{k_{4}}^{\dagger}-\delta_{k_{2} n} \delta_{k_{3} m} p_{k_{4}}^{\dagger}-\delta_{k_{2} m} \delta_{k_{4} n} p_{k_{3}}^{\dagger}+\delta_{k_{2} n} \delta_{k_{4} m} p_{k_{3}}^{\dagger}+\delta_{k_{3} m} \delta_{k_{4} n} p_{k_{2}}^{\dagger}-\delta_{k_{3} n} \delta_{k_{4} m} p_{k_{2}}^{\dagger}\right) \\
& \times\left(\delta_{l_{2} q} \delta_{l_{3} r} h_{l_{4}}^{\dagger}-\delta_{l_{2} r} \delta_{l_{3} q} h_{l_{4}}^{\dagger}-\delta_{l_{2} q} \delta_{l_{4} r} h_{l_{3}}^{\dagger}+\delta_{l_{2} r} \delta_{l_{4} q} h_{l_{3}}^{\dagger}+\delta_{l_{3} q} \delta_{l_{4} r} h_{l_{2}}^{\dagger}-\delta_{l_{3} r} \delta_{l_{4} q} h_{l_{2}}^{\dagger}\right)|\phi\rangle
\end{aligned}
$$

Now, each pair of terms ending with the same operator gives the same contribution, which yields

$$
\begin{aligned}
& \left\langle\phi_{j_{1} j_{2}}^{i_{1} i_{2}}\left|T \hat{H}^{\phi} T^{3}\right| \phi\right\rangle \\
& =-\frac{1}{6} \sum_{m n q r k_{1} l_{1} k_{2} l_{2} k_{3} l_{3} k_{4} l_{4}} V_{m n q r} c_{l_{2} l_{3} l_{4}}^{k_{2} k_{3} k_{4}}\langle\phi| h_{j_{2}} p_{i_{2}} h_{j_{1}} p_{i_{1}} p_{k_{1}}^{\dagger} h_{l_{1}}^{\dagger} \hat{D}_{l_{1}}^{k_{1}} \\
& \times\left(\delta_{k_{2} m} \delta_{k_{3} n} p_{k_{4}}^{\dagger}-\delta_{k_{2} m} \delta_{k_{4} n} p_{k_{3}}^{\dagger}+\delta_{k_{3} m} \delta_{k_{4} n} p_{k_{2}}^{\dagger}\right)\left(\delta_{l_{2} q} \delta_{l_{3} r} h_{l_{4}}^{\dagger}-\delta_{l_{2} q} \delta_{l_{4} r} h_{l_{3}}^{\dagger}+\delta_{l_{3} q} \delta_{l_{4} r} h_{l_{2}}^{\dagger}\right)|\phi\rangle \\
& =-\frac{1}{6} \sum_{m n q r k_{1} l_{1} k_{2} l_{2} k_{3} l_{3} k_{4} l_{4}} V_{m n q r} c_{l_{2} l_{3} l_{4}}^{k_{2} k_{3} k_{4}}\langle\phi| h_{j_{2}} p_{i_{2}} h_{j_{1}} p_{i_{1}} p_{k_{1}}^{\dagger} h_{l_{1}}^{\dagger} \hat{D}_{l_{1}}^{k_{1}} \\
& \times\left(\delta_{k_{2} m} \delta_{k_{3} n} \delta_{l_{2} q} \delta_{l_{3} r} p_{k_{4}}^{\dagger} h_{l_{4}}^{\dagger}-\delta_{k_{2} m} \delta_{k_{3} n} \delta_{l_{2} q} \delta_{l_{4} r} p_{k_{4}}^{\dagger} h_{l_{3}}^{\dagger}+\delta_{k_{2} m} \delta_{k_{3} n} \delta_{l_{3} q} \delta_{l_{4} r} p_{k_{4}}^{\dagger} h_{l_{2}}^{\dagger}\right. \\
& -\delta_{k_{2} m} \delta_{k_{4} n} \delta_{l_{2} q} \delta_{l_{3} r} p_{k_{3}}^{\dagger} h_{l_{4}}^{\dagger}+\delta_{k_{2} m} \delta_{k_{4} n} \delta_{l_{2} q} \delta_{l_{4} r} p_{k_{3}}^{\dagger} h_{l_{3}}^{\dagger}-\delta_{k_{2} m} \delta_{k_{4} n} \delta_{l_{3} q} \delta_{l_{4} r} p_{k_{3}}^{\dagger} h_{l_{2}}^{\dagger} \\
& \left.+\delta_{k_{3} m} \delta_{k_{4} n} \delta_{l_{2} q} \delta_{l_{3} r} p_{k_{2}}^{\dagger} h_{l_{4}}^{\dagger}-\delta_{k_{3} m} \delta_{k_{4} n} \delta_{l_{2} q} \delta_{l_{4} r} p_{k_{2}}^{\dagger} h_{l_{3}}^{\dagger}+\delta_{k_{3} m} \delta_{k_{4} n} \delta_{l_{3} q} \delta_{l_{4} r} p_{k_{2}}^{\dagger} h_{l_{2}}^{\dagger}\right)|\phi\rangle \\
& =-\frac{1}{12} \sum_{m n q r k_{1} l_{1}} V_{m n q r}\langle\phi| h_{j_{2}} p_{i_{2}} h_{j_{1}} p_{i_{1}} p_{k_{1}}^{\dagger} h_{l_{1}}^{\dagger} \\
& \times\left(\sum_{k_{4} l_{4}} c_{q r l_{4}}^{m n k_{4}} \frac{c_{l_{1} l_{4}}^{k_{1} k_{4}}}{c_{l_{4}}^{k_{4}}} p_{k_{4}}^{\dagger} h_{l_{4}}^{\dagger}-\sum_{l_{3} k_{4}} c_{q l_{3} r}^{m n k_{4}} \frac{c_{l_{1} l_{3}}^{k_{1} k_{4}}}{c_{l_{3}}^{k_{4}}} p_{k_{4}}^{\dagger} h_{l_{3}}^{\dagger}+\sum_{k_{4} l_{2}} c_{l_{2} q r}^{m n k_{4}} \frac{c_{l_{1} l_{2}}^{k_{1} k_{4}}}{c_{l_{2}}^{k_{4}}} p_{k_{4}}^{\dagger} h_{l_{2}}^{\dagger}\right. \\
& -\sum_{k_{3} l_{4}} c_{q r l_{4}}^{m k_{3} n} \frac{c_{l_{1} l_{4}}^{k_{1} k_{3}}}{c_{l_{4}}^{k_{3}}} p_{k_{3}}^{\dagger} h_{l_{4}}^{\dagger}+\sum_{k_{3} l_{3}} c_{q l_{3} r}^{m k_{3} n} \frac{c_{l_{1} l_{3}}^{k_{1} k_{3}}}{c_{l_{3}}^{k_{3}}} p_{k_{3}}^{\dagger} h_{l_{3}}^{\dagger}-\sum_{k_{3} l_{2}} c_{l_{2} q r}^{m k_{3} n} \frac{c_{l_{1} l_{2}}^{k_{1} k_{3}}}{c_{l_{2}}^{k_{3}}} p_{k_{3}}^{\dagger} h_{l_{2}}^{\dagger} \\
& \left.+\sum_{k_{2} l_{4}} c_{q r l_{4}}^{k_{2} m n} \frac{c_{l_{1} l_{4}}^{k_{1} k_{2}}}{c_{l_{4}}^{k_{2}}} p_{k_{2}}^{\dagger} h_{l_{4}}^{\dagger}-\sum_{k_{2} l_{3}} c_{q l_{3} r}^{k_{2} m n} \frac{c_{l_{1} l_{3}}^{k_{1} k_{2}}}{c_{l_{3}}^{k_{2}}} p_{k_{2}}^{\dagger} h_{l_{3}}^{\dagger}+\sum_{k_{2} l_{2}} c_{l_{2} q r}^{k_{2} m n} \frac{c_{l_{1} l_{2}}^{k_{2} k_{2}}}{c_{l_{2}}^{k_{2}}} p_{k_{2}}^{\dagger} h_{l_{2}}^{\dagger}\right)|\phi\rangle \\
& =-\frac{3}{4} \sum_{m n q r k_{1} l_{1} k_{2} l_{2}} V_{m n q r} c_{q r l_{2}}^{m n k_{2}} \frac{c_{l_{1} l_{2}}^{k_{1} k_{2}}}{c_{l_{2}}^{k_{2}}}\left\langle\phi\left|h_{j_{2}} p_{i_{2}} h_{j_{1}} p_{i_{1}} p_{k_{1}}^{\dagger} h_{l_{1}}^{\dagger} p_{k_{2}}^{\dagger} h_{l_{2}}^{\dagger}\right| \phi\right\rangle
\end{aligned}
$$




$$
\begin{aligned}
\left\langle\phi_{j_{1} j_{2}}^{i_{1} i_{2}}\left|T \hat{H}^{\phi} T^{3}\right| \phi\right\rangle & =-\frac{3}{4} \sum_{m n q r k_{1} l_{1} k_{2} l_{2}} V_{m n q r} c_{q r l_{2}}^{m n k_{2}} \frac{c_{l_{1} l_{2}}^{k_{1} k_{2}}}{c_{l_{2}}^{k_{2}}}\left\langle\phi\left|p_{i_{2}} p_{i_{1}} p_{k_{1}}^{\dagger} p_{k_{2}}^{\dagger} h_{j_{2}} h_{j_{1}} h_{l_{1}}^{\dagger} h_{l_{2}}^{\dagger}\right| \phi\right\rangle \\
& =-\frac{3}{4} \sum_{m n q r k_{1} l_{1} k_{2} l_{2}} V_{m n q r} c_{q r l_{2}}^{m n k_{2}} \frac{c_{l_{1} l_{2}}^{k_{1} k_{2}}}{c_{l_{2}}^{k_{2}}}\left(\delta_{k_{1} i_{1}} \delta_{k_{2} i_{2}}-\delta_{k_{1} i_{2}} \delta_{k_{2} i_{1}}\right)\left(\delta_{l_{1} j_{1}} \delta_{l_{2} j_{2}}-\delta_{l_{1} j_{2}} \delta_{l_{2} j_{1}}\right) \\
& =-\frac{3}{4} \sum_{m n q r} V_{m n q r}\left(c_{q r j_{2}}^{m n j_{2}} \frac{c_{j_{1} j_{2}}^{i_{1} i_{2}}}{c_{j_{2}}^{i_{2}}}-c_{q r j_{1}}^{m n i_{2}} \frac{c_{j_{2} j_{1}}^{i_{1} i_{2}}}{c_{j_{1}}^{i_{2}}}-c_{q r j_{2}}^{m n n j_{1}} \frac{c_{j_{1} j_{2}}^{i_{2} i_{1}}}{c_{j_{2}}^{i_{1}}}+c_{q r j_{1}}^{m n i_{1}} \frac{c_{j_{2} j_{1}}^{i_{2} i_{1}}}{c_{j_{1}}^{i_{1}}}\right) \\
& =-\frac{3}{4} c_{j_{1} j_{2}}^{i_{1} i_{2}} \sum_{m n q r} V_{m n q r}\left(\frac{c_{q r j_{2}}^{m n i_{2}}}{c_{l_{2}}^{i_{2}}}+\frac{c_{q r j_{1}}^{m n i_{2}}}{c_{j_{1}}^{i_{2}}}+\frac{c_{q r j_{2}}^{m n i_{1}}}{c_{j_{2}}^{i_{1}}}+\frac{c_{q r j_{1}}^{m n i_{1}}}{c_{j_{1}}^{i_{1}}}\right)
\end{aligned}
$$

and

$$
-\frac{1}{3 !}\left\langle\phi_{j_{1} j_{2}}^{i_{1} i_{2}}\left|T \hat{H}^{\phi} T^{3}\right| \phi\right\rangle=\frac{1}{8} c_{j_{1} j_{2}}^{i_{1} i_{2}} \sum_{m n q r} V_{m n q r}\left(\frac{c_{q r j_{2}}^{m n i_{2}}}{c_{l_{2}}^{i_{2}}}+\frac{c_{q r j_{1}}^{m n i_{2}}}{c_{j_{1}}^{i_{2}}}+\frac{c_{q r j_{2}}^{m n i_{1}}}{c_{j_{2}}^{i_{1}}}+\frac{c_{q r j_{1}}^{m n i_{1}}}{c_{j_{1}}^{i_{1}}}\right)
$$

$$
\begin{aligned}
\left\langle\phi_{j_{1} j_{2}}^{i_{1} i_{2}}\left|T^{2} \hat{H}^{\phi} T^{2}\right| \phi\right\rangle & =\left\langle\phi_{j_{1} j_{2}}^{i_{1} i_{2}}\left|T^{2}\right| \phi\right\rangle\left\langle\phi\left|\hat{H}^{\phi} T^{2}\right| \phi\right\rangle \\
& =2 c_{j_{1} j_{2}}^{i_{1} i_{2}}\left\langle\phi\left|\frac{1}{4} \sum_{k l m n} V_{k l m n} h_{n} h_{m} p_{l} p_{k}\left(\frac{1}{2}\right) \sum_{q r s t} c_{r t}^{q s} p_{q}^{\dagger} h_{r}^{\dagger} p_{s}^{\dagger} h_{t}^{\dagger}\right| \phi\right\rangle \\
& =\frac{1}{4} c_{j_{1} j_{2}}^{i_{1} i_{2}} \sum_{k l m n q r s t} V_{k l m n} c_{r t}^{q s}\left\langle\phi\left|h_{n} h_{m} p_{l} p_{k} p_{q}^{\dagger} h_{r}^{\dagger} p_{s}^{\dagger} h_{t}^{\dagger}\right| \phi\right\rangle \\
& =-\frac{1}{4} c_{j_{1} j_{2}}^{i_{1} i_{2}} \sum_{k l m n q r s t} V_{k l m n} c_{r t}^{q s}\left\langle\phi\left|p_{l} p_{k} p_{q}^{\dagger} p_{s}^{\dagger} h_{n} h_{m} h_{r}^{\dagger} h_{t}^{\dagger}\right| \phi\right\rangle \\
& =-c_{j_{1} j_{2}}^{i_{1} i_{2}} \sum_{k l m n} V_{k l m n} c_{m n}^{k l},
\end{aligned}
$$

where we have used the result of 1.27 for $\left\langle\phi_{j_{1} j_{2}}^{i_{1} i_{2}}\left|T^{2}\right| \phi\right\rangle$, thus

$$
\frac{1}{4}\left\langle\phi_{j_{1} j_{2}}^{i_{1} i_{2}}\left|T^{2} \hat{H}^{\phi} T^{2}\right| \phi\right\rangle=-\frac{1}{4} c_{j_{1} j_{2}}^{i_{1} i_{2}} \sum_{k l m n} V_{k l m n} c_{m n}^{k l}
$$


Finally, after adding and rearranging the terms, equation (1.4) becomes

$$
\begin{aligned}
& 0=-V_{i_{1} i_{2} j_{1} j_{2}}+t_{i_{1} j_{1}}^{\phi} c_{j_{2}}^{i_{2}}-t_{i_{1} j_{2}}^{\phi} c_{j_{1}}^{i_{2}}-t_{i_{2} j_{1}}^{\phi} c_{j_{2}}^{i_{1}}+t_{i_{2} j_{2}}^{\phi} c_{j_{1}}^{i_{1}}-\sum_{k}\left(V_{i_{1} i_{2} j_{1} k} c_{j_{2}}^{k}-V_{i_{1} i_{2} j_{2} k} c_{j_{1}}^{k}\right) \\
& +\sum_{k}\left(V_{j_{1} j_{2} i_{1} k} c_{k}^{i_{2}}-V_{j_{1} j_{2} i_{2} k} c_{k}^{i_{1}}\right)+\sum_{k}\left(t_{i_{1} k}^{\phi} c_{j_{1} j_{2}}^{k i_{2}}+t_{i_{2} k}^{\phi} c_{j_{1} j_{2}}^{i_{1} k}\right)-\sum_{k}\left(t_{j_{1} k}^{\phi} c_{k j_{2}}^{i_{1} i_{2}}+t_{j_{2} k}^{\phi} c_{j_{1} k}^{i_{1} i_{2}}\right) \\
& -\frac{1}{2} \sum_{k l} V_{i_{1} i_{2} k l} c_{j_{1} j_{2}}^{k l}-\frac{1}{2} \sum_{k l} V_{j_{1} j_{2} k l} c_{k l}^{i_{1} i_{2}}-\sum_{k l}\left(V_{i_{1} k j_{1} l} c_{k j_{2}}^{l i_{2}}+V_{i_{2} k j_{1} l} c_{k j_{2}}^{i_{1} l}+V_{i_{1} k j_{2} l} c_{j_{1} k}^{l i_{2}}+V_{i_{2} k j_{2} l} c_{j_{1} k}^{i_{1} l}\right) \\
& +\sum_{k l} t_{k l}^{\phi} c_{l j_{1} j_{2}}^{k i_{1} i_{2}}-\frac{1}{2} \sum_{k l m}\left(V_{k l m i_{1}} c_{m j_{1} j_{2}}^{k l i_{2}}-V_{k l m i_{2}} c_{m j_{1} j_{2}}^{k l i_{1}}\right)+\frac{1}{2} \sum_{k l m}\left(V_{k l m j_{1}} c_{k l j_{2}}^{m i_{1} i_{2}}-V_{k l m j_{2}} c_{k l j_{1}}^{m i_{1} i_{2}}\right) \\
& +c_{j_{1} j_{2}}^{i_{1} i_{2}} \sum_{k l} t_{k l}^{\phi} c_{l}^{k}-\frac{1}{4} \sum_{k l m n} V_{k l m n} c_{m n j_{1} j_{2}}^{k l l_{1} i_{2}}-\frac{1}{4} c_{j_{1} j_{2}}^{i_{1} i_{2}} \sum_{k l m n} V_{k l m n} c_{m n}^{k l} \\
& +c_{j_{1} j_{2}}^{i_{1} i_{2}}\left[-\frac{1}{2}\left(\frac{t_{i_{2} j_{2}}^{\phi}}{c_{j_{2}}^{i_{2}}}+\frac{t_{i_{1} j_{2}}^{\phi}}{c_{j_{2}}^{i_{1}}}+\frac{t_{i_{2} j_{1}}^{\phi}}{c_{j_{1}}^{i_{2}}}+\frac{t_{i_{1} j_{1}}^{\phi}}{c_{j_{1}}^{i_{1}}}\right)-\frac{1}{2} \sum_{k}\left(\frac{t_{i_{2} k}^{\phi} c_{j_{2}}^{k}}{c_{j_{2}}^{i_{2}}}+\frac{t_{i_{1} k}^{\phi} c_{j_{2}}^{k}}{c_{j_{2}}^{i_{1}}}+\frac{t_{i_{2} k}^{\phi} c_{j_{1}}^{k}}{c_{j_{1}}^{i_{2}}}+\frac{t_{i_{1} k}^{\phi} c_{j_{1}}^{k}}{c_{j_{1}}^{i_{1}}}\right)\right. \\
& +\frac{1}{2} \sum_{k}\left(\frac{t_{j_{2} k}^{\phi} c_{k}^{i_{2}}}{c_{j_{2}}^{i_{2}}}+\frac{t_{j_{2} k}^{\phi} c_{k}^{i_{1}}}{c_{j_{2}}^{i_{1}}}+\frac{t_{j_{1}}^{\phi} c_{k}^{i_{2}}}{c_{j_{1}}^{i_{2}}}+\frac{t_{j_{1} k}^{\phi} c_{k}^{i_{1}}}{c_{j_{1}}^{i_{1}}}\right)+\frac{1}{2} \sum_{k l}\left(\frac{V_{i_{2} l j_{2} k} c_{l}^{k}}{c_{j_{2}}^{i_{2}}}+\frac{V_{i_{1} l j_{2} k} c_{l}^{k}}{c_{j_{2}}^{i_{1}}}+\frac{V_{i_{2} l j_{1} k} c_{l}^{k}}{c_{j_{1}}^{i_{2}}}+\frac{V_{i_{1} l j_{1} k} c_{l}^{k}}{c_{j_{1}}^{i_{1}}}\right) \\
& -\sum_{k l} t_{k l}^{\phi}\left(\frac{c_{l j_{2}}^{k i_{2}}}{c_{j_{2}}^{i_{2}}}+\frac{c_{l j_{2}}^{k i_{1}}}{c_{j_{2}}^{i_{1}}}+\frac{c_{l j_{1}}^{k i_{2}}}{c_{j_{1}}^{i_{2}}}+\frac{c_{l j_{1}}^{k i_{1}}}{c_{j_{1}}^{i_{1}}}\right)+\frac{1}{2} \sum_{k l m}\left(\frac{V_{k l m i_{2}} c_{m j_{2}}^{k l}}{c_{j_{2}}^{i_{2}}}+\frac{V_{k l m i_{1}} c_{m j_{2}}^{k l}}{c_{j_{2}}^{i_{1}}}+\frac{V_{k l m i_{2}} c_{m j_{1}}^{k l}}{c_{j_{1}}^{i_{2}}}+\frac{V_{k l m i_{1}} c_{m j_{1}}^{k l}}{c_{j_{1}}^{i_{1}}}\right) \\
& \left.-\frac{1}{2} \sum_{k l m}\left(\frac{V_{k l m j_{2}} c_{k l}^{m i_{2}}}{c_{j_{2}}^{i_{2}}}+\frac{V_{k l m j_{2}} c_{k l}^{m i_{1}}}{c_{j_{2}}^{i_{1}}}+\frac{V_{k l m j_{1}} c_{k l}^{m i_{2}}}{c_{j_{1}}^{i_{2}}}+\frac{V_{k l m j_{1}} c_{k l}^{m i_{1}}}{c_{j_{1}}^{i_{1}}}\right)+\frac{1}{8} \sum_{k l m n} V_{k l m n}\left(\frac{c_{m n n j_{2}}^{k l i_{2}}}{c_{l_{2}}^{i_{2}}}+\frac{c_{m n j_{1}}^{k l l_{2}}}{c_{j_{1}}^{i_{2}}}+\frac{c_{m n j_{2}}^{k l i_{1}}}{c_{j_{2}}^{i_{1}}}+\frac{c_{m n j_{1}}^{k l i_{1}}}{c_{j_{1}}^{i_{1}}}\right)\right] .
\end{aligned}
$$


or, after multiplying by $c_{j_{1}}^{i_{1}} c_{j_{2}}^{i_{1}} c_{j_{1}}^{i_{2}} c_{j_{2}}^{i_{2}}$,

$$
\begin{aligned}
& 0=c_{j_{1}}^{i_{1}} c_{j_{2}}^{i_{1}} c_{j_{1}}^{i_{2}} c_{j_{2}}^{i_{2}}\left[-V_{i_{1} i_{2} j_{1} j_{2}}+t_{i_{1} j_{1}}^{\phi} c_{j_{2}}^{i_{2}}-t_{i_{1} j_{2}}^{\phi} c_{j_{1}}^{i_{2}}-t_{i_{2} j_{1}}^{\phi} c_{j_{2}}^{i_{1}}+t_{i_{2} j_{2}}^{\phi} c_{j_{1}}^{i_{1}}-\sum_{k}\left(V_{i_{1} i_{2} j_{1} k} c_{j_{2}}^{k}-V_{i_{1} i_{2} j_{2} k} c_{j_{1}}^{k}\right)\right. \\
& +\sum_{k}\left(V_{j_{1} j_{2} i_{1} k} c_{k}^{i_{2}}-V_{j_{1} j_{2} i_{2} k} c_{k}^{i_{1}}\right)+\sum_{k}\left(t_{i_{1} k}^{\phi} c_{j_{1} j_{2}}^{k i_{2}}+t_{i_{2} k}^{\phi} c_{j_{1} j_{2}}^{i_{1} k}\right)-\sum_{k}\left(t_{j_{1} k}^{\phi} c_{k j_{2}}^{i_{1} i_{2}}+t_{j_{2} k}^{\phi} c_{j_{1} k}^{i_{1} i_{2}}\right) \\
& -\frac{1}{2} \sum_{k l} V_{i_{1} i_{2} k l} c_{j_{1} j_{2}}^{k l}-\frac{1}{2} \sum_{k l} V_{j_{1} j_{2} k l} c_{k l}^{i_{1} i_{2}}-\sum_{k l}\left(V_{i_{1} k j_{1} l} c_{k j_{2}}^{l i i_{2}}+V_{i_{2} k j_{1} l} c_{k j_{2}}^{i_{1} l}+V_{i_{1} k j_{2} l} c_{j_{1} k}^{l i_{2}}+V_{i_{2} k j_{2} l} c_{j_{1} k}^{i_{1} l}\right) \\
& +\sum_{k l} t_{k l}^{\phi} c_{l j_{1} j_{2}}^{k i_{1} i_{2}}-\frac{1}{2} \sum_{k l m}\left(V_{k l m i_{1}} c_{m j_{1} j_{2}}^{k l i_{2}}-V_{k l m i_{2}} c_{m j_{1} j_{2}}^{k l i_{1}}\right)+\frac{1}{2} \sum_{k l m}\left(V_{k l m j_{1}} c_{k l j_{2}}^{m i_{1} i_{2}}-V_{k l m j_{2}} c_{k l j_{1}}^{m i_{1} i_{2}}\right) \\
& \left.+c_{j_{1} j_{2}}^{i_{1} i_{2}} \sum_{k l} t_{k l}^{\phi} c_{l}^{k}-\frac{1}{4} \sum_{k l m n} V_{k l m n} c_{m n j_{1} j_{2}}^{k l i_{1} i_{2}}-\frac{1}{4} c_{j_{1} j_{2}}^{i_{1} i_{2}} \sum_{k l m n} V_{k l m n} c_{m n}^{k l}\right] \\
& +c_{j_{1} j_{2}}^{i_{1} i_{2}}\left[-\frac{1}{2}\left(t_{i_{2} j_{2}}^{\phi} c_{j_{1}}^{i_{1}} c_{j_{2}}^{i_{1}} c_{j_{1}}^{i_{2}}+t_{i_{1} j_{2}}^{\phi} c_{j_{1}}^{i_{1}} c_{j_{1}}^{i_{2}} c_{j_{2}}^{i_{2}}+t_{i_{2} j_{1}}^{\phi} c_{j_{1}}^{i_{1}} c_{j_{2}}^{i_{1}} c_{j_{2}}^{i_{2}}+t_{i_{1} j_{1}}^{\phi} c_{j_{2}}^{i_{1}} c_{j_{1}}^{i_{2}} c_{j_{2}}^{i_{2}}\right)\right. \\
& -\frac{1}{2} \sum_{k}\left(t_{i_{2} k}^{\phi} c_{j_{2}}^{k} c_{j_{1}}^{i_{1}} c_{j_{2}}^{i_{1}} c_{j_{1}}^{i_{2}}+t_{i_{1} k}^{\phi} c_{j_{2}}^{k} c_{j_{1}}^{i_{1}} c_{j_{1}}^{i_{2}} c_{j_{2}}^{i_{2}}+t_{i_{2} k}^{\phi} c_{j_{1}}^{k} c_{j_{1}}^{i_{1}} c_{j_{2}}^{i_{1}} c_{j_{2}}^{i_{2}}+t_{i_{1} k}^{\phi} c_{j_{1}}^{k} c_{j_{2}}^{i_{1}} c_{j_{1}}^{i_{2}} c_{j_{2}}^{i_{2}}\right) \\
& +\frac{1}{2} \sum_{k}\left(t_{j_{2} k}^{\phi} c_{k}^{i_{2}} c_{j_{1}}^{i_{1}} c_{j_{2}}^{i_{1}} c_{j_{1}}^{i_{2}}+t_{j_{2}}^{\phi} c_{k}^{i_{1}} c_{j_{1}}^{i_{1}} c_{j_{1}}^{i_{2}} c_{j_{2}}^{i_{2}}+t_{j_{1} k}^{\phi} c_{k}^{i_{2}} c_{j_{1}}^{i_{1}} c_{j_{2}}^{i_{1}} c_{j_{2}}^{i_{2}}+t_{j_{1} k}^{\phi} c_{k}^{i_{1}} c_{j_{2}}^{i_{1}} c_{j_{1}}^{i_{2}} c_{j_{2}}^{i_{2}}\right) \\
& +\frac{1}{2} \sum_{k l}\left(V_{i_{2} l j_{2} k} c_{l}^{k} c_{j_{1}}^{i_{1}} c_{j_{2}}^{i_{1}} c_{j_{1}}^{i_{2}}+V_{i_{1} l j_{2} k} c_{l}^{k} c_{j_{1}}^{i_{1}} c_{j_{1}}^{i_{2}} c_{j_{2}}^{i_{2}}+V_{i_{2} l j_{1} k} c_{l}^{k} c_{j_{1}}^{i_{1}} c_{j_{2}}^{i_{1}} c_{j_{2}}^{i_{2}}+V_{i_{1} l j_{1} k} c_{l}^{k} c_{j_{2}}^{i_{1}} c_{j_{1}}^{i_{2}} c_{j_{2}}^{i_{2}}\right) \\
& -\sum_{k l} t_{k l}^{\phi}\left(c_{l j_{2}}^{k i_{2}} c_{j_{1}}^{i_{1}} c_{j_{2}}^{i_{1}} c_{j_{1}}^{i_{2}}+c_{l j_{2}}^{k i_{1}} c_{j_{1}}^{i_{1}} c_{j_{1}}^{i_{2}} c_{j_{2}}^{i_{2}}+c_{l j_{1}}^{k i_{2}} c_{j_{1}}^{i_{1}} c_{j_{2}}^{i_{1}} c_{j_{2}}^{i_{2}}+c_{l j_{1}}^{k i_{1}} c_{j_{2}}^{i_{1}} c_{j_{1}}^{i_{2}} c_{j_{2}}^{i_{2}}\right) \\
& +\frac{1}{2} \sum_{k l m}\left(V_{k l m i_{2}} c_{m j_{2}}^{k l} c_{j_{1}}^{i_{1}} c_{j_{2}}^{i_{1}} c_{j_{1}}^{i_{2}}+V_{k l m i_{1}} c_{m j_{2}}^{k l} c_{j_{1}}^{i_{1}} c_{j_{1}}^{i_{2}} c_{j_{2}}^{i_{2}}+V_{k l m i_{2}} c_{m j_{1}}^{k l} c_{j_{1}}^{i_{1}} c_{j_{2}}^{i_{1}} c_{j_{2}}^{i_{2}}+V_{k l m i_{1}} c_{m j_{1}}^{k l} c_{j_{2}}^{i_{1}} c_{j_{1}}^{i_{2}} c_{j_{2}}^{i_{2}}\right) \\
& -\frac{1}{2} \sum_{k l m}\left(V_{k l m j_{2}} c_{k l}^{m i_{2}} c_{j_{1}}^{i_{1}} c_{j_{2}}^{i_{1}} c_{j_{1}}^{i_{2}}+V_{k l m j_{2}} c_{k l}^{m i_{1}} c_{j_{1}}^{i_{1}} c_{j_{1}}^{i_{2}} c_{j_{2}}^{i_{2}}+V_{k l m j_{1}} c_{k l}^{m i_{2}} c_{j_{1}}^{i_{1}} c_{j_{2}}^{i_{1}} c_{j_{2}}^{i_{2}}+V_{k l m j_{1}} c_{k l}^{m i_{1}} c_{j_{2}}^{i_{1}} c_{j_{1}}^{i_{2}} c_{j_{2}}^{i_{2}}\right) \\
& \left.+\frac{1}{8} \sum_{k l m n} V_{k l m n}\left(c_{m n j_{2}}^{k l i_{2}} c_{j_{1}}^{i_{1}} c_{j_{2}}^{i_{1}} c_{j_{1}}^{i_{2}}+c_{m n j_{1}}^{k l i_{j_{1}}} c_{j_{1}}^{i_{1}} c_{j_{2}}^{i_{1}} c_{j_{2}}^{i_{2}}+c_{m n j_{2}}^{k l i_{1}} c_{j_{1}}^{i_{1}} c_{j_{1}}^{i_{2}} c_{j_{2}}^{i_{2}}+c_{m n j_{1}}^{k l i_{1}} c_{j_{2}}^{i_{1}} c_{j_{1}}^{i_{2}} c_{j_{2}}^{i_{2}}\right)\right]
\end{aligned}
$$

